\title{
Geometry of Kähler Metrics and Foliations by Holomorphic Discs
}

\author{
X. X. Chen and G. Tian* \\ Dedicated to Professor E. Calabi for his 80th birthday
}

\section{Contents}

1 Introduction and Main Results

1.1 A brief Tour of Extremal Kähler Metrics . . . . . . . . . . . . . . . . 2

1.2 Space of Kähler Metrics. . . . . . . . . . . . . . . . . . . . . . 4

1.3 An Approach to Uniqueness and lower bound for the K-energy . . . . . . . . . 6

1.4 Ideas for Proof of Theorem $1.3 .4 \ldots \ldots \ldots$

1.5 Organization ........................... 11

2 Foliations and the Homogenous comlex Monge Ampere Equation 11

2.1 Semmes' construction . . . . . . . . . . . . . . . . . . . . 12

2.2 Local Uniqueness for Equation (1.1) . . . . . . . . . . . . . . . . . . 12

2.3 Almost Smooth Solutions $\Leftrightarrow$ Nearly Smooth Foliations . . . . . . . . . . . 13

3 Deformation of holomorphic disks with totally real boundary 20

3.1 Local analysis of holomorphic disks . . . . . . . . . . . . . . . . . . 20

3.2 The universal moduli space is regular . . . . . . . . . . . . . . 22

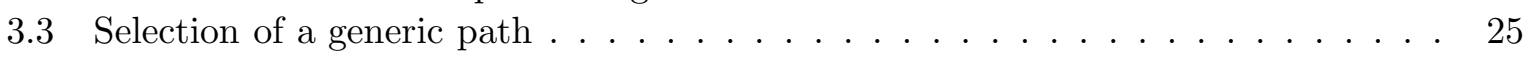

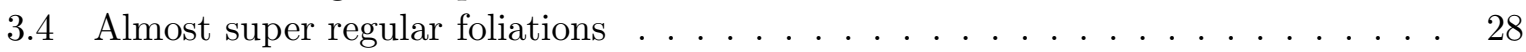

3.4.1 Open-denseness of almost super regular disks . . . . . . . . . . 30

3.4.2 Almost super regular foliations along a generic path . . . . . . . . . 34

4 Basic curvature equations along leaves 37

4.1 Introduction . . . . . . . . . . . . . . . . . . . . . . . 37

4.2 Hermitian Curvature formulas . . . . . . . . . . . . . . . . 38

${ }^{*}$ Both authors are supported by NSF research grants and the second author was also partly supported by a J. Simons fund 
5 Compactness of holomorphic disks

5.1 Proof of Theorem 5.0.16 . . . . . . . . . . . . . . . . 45

5.2 No vertical bubble-Proof of Theorem 5.0.14 . . . . . . . . . . . . . . . . . 47

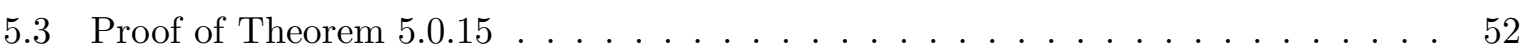

5.3.1 Uniform $C^{1}$ transversal derivatives of almost super regular foliations . . . 52

5.3.2 The Limit of Super-regular disks with finite capacity is Super regular . . 57

5.4 Proof of Theorem 5.0.17. Compactness of almost super regular foliations . . . . . 59

6 The (modified) K energy along almost solutions $\quad 62$

6.1 The Subharmonicity of the K energy . . . . . . . . . . . . . . . 62

6.2 The lower bound of the (modified) K energy . . . . . . . . . . . . . . 69

7 Partial regularity of the K energy minimzer

7.1 Strong convergence lemma for volume form . . . . . . . . . . . . . . . . . 71

7.2 Special properties of the $\mathrm{K}$ energy minimizer . . . . . . . . . . . . 73

7.3 A regularity theorem for a $C^{1,1}$ minimizer of the $\mathrm{K}$ energy functional and the weak "Kähler Ricci" flow . . . . . . . . . . . . . . . . . 80

8 The Problem of uniqueness of extremal Käher metrics

9 Appendix: Loop space of $G l(n, c)$ and holomorphic discs 8

\section{Introduction and Main Results}

The purpose of this paper is to establish a completely new partial regularity theory on certain homogeneous complex Monge-Ampere equations. Our partial regularity theory will be obtained by studying foliations by holomorphic curves and and their relations to homogeneous complex Monge-Ampere equations. As applications, we will prove the uniqueness of extremal Kähler metrics and give an necessary condition for existence of extremal Kähler metrics. Further applications will be discussed in our forthcoming papers.

\subsection{A brief Tour of Extremal Kähler Metrics}

Following [4, we call a Kähler metric extremal if the complex gradient of its scalar curvature is a holomorphic vector field. In particular, any Kähler metric of constant scalar curvature is extremal, conversely, if the underlying Kähler manifold has no holomorphic vector fields, then an extremal Kähler metric is of constant scalar curvature. It follows from the standard Hodge theory that a Kähler metric of constant scalar curvature becomes Kähler-Einstein if its Kähler class is propotional to the first Chern class of the underlying manifold. 
In the 50's, E. Calabi proposed the problem of studying existence of Kähler-Einstein metrics on compact Kähler manifolds with definite first Chern class. In 1976, S. T. Yau solved the famous Calabi conjecture. A corollary of this implies that any Kähler manifold with vanishing first Chern class has a Calabi-Yau metric, that is, a Ricci-flat Kähler metric. Around the same time, T. Aubin and S. T. Yau independently proved existence of Kähler-Einstein metrics on compact Kähler manifolds with negative first Chern class. The remaining case is technically more involved. In [29], the second named author proved that a complex surface with positive first Chern clas admits a Kähler-Einstein metric if and only if its automorphism group is reductive. For higher dimensions, he proved in [27] that the existence of Kähler-Einstein metrics with positive scalar curvature is equivalent to an analytic stability of the underlying Kähler manifold. It remains open how this analytic stability is related to certain algebraic stability from geometric invariant theory (cf. [27, 30, 11, 24, 25]). There has not been much progress made on the existence of general extremal metrics, even in the case of complex surfaces. One possible reason is that the corresponding equation is highly nonlinear and of 4 th order. We will give an necessary condition for existence of extremal metrics.

On the other hand, there have been many results on uniqueness of extremal metrics. Using the maximum principle, E. Calabi observed in 50's that Kähler-Einstein metrics of non-positive scalar curvature are unique in their Kähler class. In 1, Bando and Mabuchi proved uniqueness of Kähler-Einstein metric of positive scalar curvature modulo holomorphic automorphisms. In 31, X.H. Zhu and the second named author proved uniqueness of Kähler-Ricci Solitons on any Kähler manifolds with positive first Chern class. Following a suggestion of Donaldson, the first named author proved in [8] uniqueness for Kähler metrics of constant scalar curvature in any Kähler class when the underlying manifold has non-positive first Chern class. In [12, S. Donaldson proved that Kähler metrics of constant scalar curvature are unique in any rational Kähler class on any projective manifolds without non-trivial holomorphic vector fields. ${ }^{1}$

In this paper, we will prove

Theorem 1.1.1. Let $(M, \Omega)$ be a compact Kähler manifold with a Kähler class $\Omega \in H^{2}(M, \mathbb{R}) \cap$ $H^{1,1}(M, \mathbb{C})$. Then there is at most one extremal Kähler metric with Kähler class $\Omega$ modulo holomorphic transformations, that is, if $\omega_{1}$ and $\omega_{2}$ are extremal Kähler metrics with the same Kähler class, then there is a holomorphic transformation $\sigma$ such that $\sigma^{*} \omega_{1}=\omega_{2}$.

In order to give a necessary condition for the existence of Kähler metrics with constant scalar curvature, we recall the K-energy introduced by T. Mabuchi in 20]: For any $\phi$ with $\omega+\sqrt{-1} \partial \bar{\partial} \phi>0$, which will be denoted by $\omega_{\phi}$, define

$$
\mathbf{E}_{\omega}(\phi)=-\int_{0}^{1} \int_{M} \dot{\phi}\left(s\left(\omega_{\phi_{t}}\right)-\mu\right) \omega_{\phi_{t}}^{n} \wedge d t
$$

\footnotetext{
${ }^{1}$ During preparation of this paper, we learned from T. Mabuchi that he was able to remove the assumption on non-existence of holomorphic vector fields in the special case of projective manifolds.
} 
where $\omega_{\phi_{t}}$ is any path of Kähler metrics joining $\omega$ and $\omega_{\phi}, s\left(\omega_{\phi_{t}}\right)$ denotes the scalar curvature and $\mu$ is its average. It turns out that $\mu$ is determined by the first Chern class $c_{1}(M)$ and the Kähler class $[\omega]$.

Theorem 1.1.2. Let $M$ be a compact Kähler manifold with a constant scalar curvature Kähler metric $\omega$. Then $\mathbf{E}_{\omega}(\phi) \geq 0$ for any $\phi$ with $\omega_{\phi}>0$.

This theorem was proved for Kähler-Einstein metrics in [1] (also see [27]) and in [8] for Kähler manifolds with non-positive first Chern class. ${ }^{2}$ This theorem can be also generalized to arbitrary extremal Kähler metrics by using the modified K-energy. This theorem gives a partial answer to a conjecture of the second author: $M$ has a constant scalar curvature Kähler metric in a given Kähler class $[\omega]$ if and only if the K-energy is proper in a suitable sense on the space of Kähler metrics with the fixed Kähler class $[\omega]$. Combining Theorem 1.1.2 with results in 30] and 25], we can deduce

Corollary 1.1.3. Let $(M, L)$ be a polarized algebraic manifold, that is, $M$ is algebraic and $L$ is a positive line bundle. If there is a constant scalar curvature Kähler metric with Kähler class equal to $c_{1}(L)$, then $(M, L)$ is asymptotically $K$-semistable or CM-semistable in the sense of [27] (also see [30]) ${ }^{3}$.

\subsection{Space of Kähler Metrics.}

Now let us explain how the above theorems can be proved. First let us give a direct approach suggested by S. Donaldson and developed in [8]. Let $\omega$ be a fixed Kähler metric on $M$. It follows from Hodge theory that the space of Kähler metrics with Kähler class [ $\omega]$ can be identified with the space of Kähler potentials

$$
\mathcal{H}_{\omega}=\left\{\phi \mid \omega_{\phi}=\omega+\sqrt{-1} \partial \bar{\partial} \phi>0 \text { on } M\right\} / \sim,
$$

where $\phi_{1} \sim \phi_{2}$ if and only if $\phi_{1}=\phi_{2}+c$ for some constant $c$. We will drop the subscript $\omega$ if no possible confusion may occur. A tangent vector in $T_{\phi} \mathcal{H}$ is just a function $\phi_{0}$ such that

$$
\int_{M} \phi_{0} \omega_{\phi}^{n}=0
$$

Its norm in the $L^{2}$-metric on $\mathcal{H}$ is given by (cf. [20])

$$
\left\|\phi_{0}\right\|_{\phi}^{2}=\int_{M} \phi_{0}^{2} \omega_{\phi}^{n}
$$

\footnotetext{
${ }^{2}$ After we finished the first draft of this paper, we learned that S. Donaldson proved this theorem in the case of projective manifolds and without holomorphic vector fields 13 . His method is completely different from ours.

${ }^{3}$ According to [25], the CM-stability (semistability) is equivalent to the K-stability (semistability).
} 
A straightforward computation shows that the geodesic equation of this $L^{2}$ metric is

$$
\phi^{\prime \prime}(t)-\frac{1}{2}\left\langle d \phi^{\prime}, d \phi^{\prime}\right\rangle_{\phi}=0
$$

where $\langle\cdot, \cdot\rangle_{\phi}$ denotes the natural inner product on $T^{*} M$ induced by the Kähler metric $\omega_{\phi}$, $\phi(t) \in \mathcal{H}$ for any $t \in[0,1]$ and $\phi^{\prime}, \phi^{\prime \prime}$ denote the derivatives of $\phi$ on $t$. Set $\phi(t, \theta, x)=\phi(t)(x)$ for $t \in[0,1], \theta \in S^{1}$ and $x \in M$, then the path $\{\phi(t)\}$ satisfies the geodesic equation if and only if the function $\phi$ on $[0,1] \times S^{1} \times M$ satisfies the homogeneous complex Monge-Ampere equation

$$
\left(\pi_{2}^{*} \omega+\sqrt{-1} \partial \bar{\partial} \phi\right)^{n+1}=0, \quad \text { on } \Sigma \times M,
$$

where $\Sigma=[0,1] \times S^{1}$ and $\pi_{2}: \Sigma \times M \mapsto M$ and $\pi_{1}: \Sigma \times M \mapsto \Sigma$ are two orthogonal projections. Using the convexity of the K-energy along geodesics of the $L^{2}$-metric, S. Donaldson observed in [10] that both Theorem 1.1.1 and 1.1.2 follow if one can show: For any $\phi_{0}, \phi_{1} \in \mathcal{H}$, (1.1) has a smooth solution $\phi$ such that $\phi(t, \theta, \cdot) \in \mathcal{H}, \phi(0, \theta, \cdot)=\phi_{0}$ and $\phi(1, \theta, \cdot)=\phi_{1}$. However, this turns out to be a very difficult problem and remains open until now. In fact, one can consider (1.1) over a general Riemann surface $\Sigma$ with boundary condition $\phi=\phi_{0}$ along $\partial \Sigma$, where $\phi_{0}$ is a smooth function on $\partial \Sigma \times M$ such that $\phi_{0}(z, \cdot) \in \mathcal{H}$ for each $z \in \partial \Sigma .^{4}$ It also has geometric meaning. The equation (1.1) can be regarded as the infinite dimensional version of the WZW equation for maps from $\Sigma$ into $\mathcal{H}$ (cf. [10]..$^{5}$ The following theorem was proved by the first named author in [8] and will play a fundamental role in this paper.

Theorem 1.2.1. ([8]) For any smooth map $\phi_{0}: \partial \Sigma \rightarrow \mathcal{H}$, there exists a unique $C^{1,1}$ solution $\phi$ of (1.1) such that $\phi=\phi_{0}$ on $\partial \Sigma$ and $\phi(z, \cdot) \in \overline{\mathcal{H}}$ for each $z \in \Sigma{ }^{6}$

The lack of sufficient regularity for above solution is the obstruction to proving Theorem 1.1.1 and 1.1.2 in general cases by using geodesics. We should point out that complex Monge-Ampere equations have been studied extensively (cf. [17, [18, [2] etc.). However, the regularity for homogeneous complex Monge-Ampere equations beyond $C^{1,1}$ has been missing. Indeed, there are examples in which some solutions are only $C^{1,1}$, here is a simple example: Let $\Omega$ be the unit ball in $\mathbb{C}^{2}$ and define

$$
u=\left\{\begin{array}{llc}
0 & \text { if } & \left|z_{1}\right|^{2},\left|z_{2}\right|^{2} \leq \frac{1}{2} \\
\left(\frac{1}{2}-\left|z_{1}\right|^{2}\right)^{2} & \text { if } & \left|z_{1}\right|^{2} \geq \frac{1}{2} \\
\left(\frac{1}{2}-\left|z_{2}\right|^{2}\right)^{2} & \text { if } & \left|z_{2}\right|^{2} \geq \frac{1}{2}
\end{array}\right.
$$

then $(\partial \bar{\partial} u)^{2}=0$ on $\Omega$ and $\left.u\right|_{\partial \Omega}$ is smooth, but $u$ is only $C^{1,1}$.

\footnotetext{
${ }^{4}$ we often regard $\phi_{0}$ as a smooth map from $\partial \Sigma$ into $\mathcal{H}$.

${ }^{5}$ Original WZW equation is for maps from a Riemann surface into a Lie group.

${ }^{6}$ Here $\overline{\mathcal{H}}$ denotes the closure of $\mathcal{H}$ in any $C^{1, \alpha}(\Sigma \times M)$-topology $(\forall \alpha \in(0,1))$. A function $\varphi \in \overline{\mathcal{H}}$ if there exists a sequence of potential functions $\left\{\varphi_{m} \in \mathcal{H}, m \in \mathbb{N}\right\}$ with uniform $C^{1,1}$ bound such that $\varphi_{m} \rightarrow \varphi$ in weak $C^{1,1}$ topology.
} 
Therefore, it may not be possible to have smooth solutions for (1.1) with general boundary values. Of course, our boundary values are special and they satisfy certain positivity condition, that is, any boundary function $\phi_{0}$ takes values in $\mathcal{H}$. This fact will play a crucial role in our approach.

\subsection{An Approach to Uniqueness and lower bound for the K-energy}

Our proof of Theorem 1.1.1 and 1.1.2 starts with the following observations: Given two functions $\phi_{0}$ and $\phi_{1}$ in $\mathcal{H}$, if there is a sufficiently nice solution $\phi$, which is not necessarily smooth, of (1.1) on $[0,1] \times \mathbb{R} \times M$, then the evaluation function $f=\mathbf{E}(\phi(z, \cdot))$ is bounded, subharmonic ${ }^{7}$ and constant along each boundary component of $[0,1] \times \mathbb{R}$. Furthermore, if $\phi_{0}$ is a critical metric of $\mathbf{E}$, then it follows from the Maximum principle that $\mathbf{E}\left(\phi_{1}\right) \geq \mathbf{E}\left(\phi_{0}\right)$ and equality holds if and only if each $\phi(z, \cdot)$ is a critical metric of $\mathbf{E}$. The infinite strip $[0,1] \times \mathbb{R}$ can be approximated by bounded disks. Hence, if (1.1) has a uniformly bounded and sufficiently smooth solution when $\Sigma$ is a disk, then Theorem 1.1.1 and 1.1.2 follow.

In this paper, we will establish a partial regularity theory for (1.1) in the case that $\Sigma$ is a unit disk. This seems to be the first partial regularity theory for homogeneous complex MongeAmpere equations.

First let us introduce some notations. Suppose that $\phi$ is a $C^{1,1}$ solution of (1.1), we denote by $\mathcal{R}_{\phi}$ the set of all $(z, x) \in \Sigma \times M$ near which $\phi$ is smooth and $\omega_{\phi\left(z^{\prime}, \cdot\right)}=\omega+\sqrt{-1} \partial \bar{\partial} \phi\left(z^{\prime}, \cdot\right)$ is a Kähler metric. We may regard $\mathcal{R}_{\phi}$ as the regular set of $\phi$. It is open, but aprior, it may be empty. We have a distribution $\mathcal{D}_{\phi} \subset T(\Sigma \times M)$ over $\mathcal{R}_{\phi}$ :

$$
\left.\mathcal{D}_{\phi}\right|_{(z, x)}=\left\{v \in T_{z} \Sigma \times T_{x} M \mid i_{v}\left(\pi_{2}^{*} \omega+\sqrt{-1} \partial \bar{\partial} \phi\right)=0\right\}, \quad(z, x) \in \mathcal{R}_{\phi} .
$$

Here $i_{v}$ denotes the interior product. Since the form is closed, $\mathcal{D}_{\phi}$ is integrable. We say that $\mathcal{R}_{\phi}$ is saturated in $\mathcal{V} \subset \Sigma \times M$ if every maximal integral sub-manifold of $\mathcal{D}_{\phi}$ in $\mathcal{R}_{\phi} \cap \mathcal{V}$ is a disk and closed in $\mathcal{V}$. By nature of product manifold, we may write any vector in $\mathcal{D}_{\phi}$ as (after re-scaling)

$$
\frac{\partial}{\partial z}+\left.X \in \mathcal{D}_{\phi}\right|_{(z, x)}, \quad \text { where } X \in T_{x}^{1,0} M .
$$

Definition 1.3.1. A solution $\phi$ of (1.1) is called partially smooth if it is $C^{1,1}$-bounded on $\Sigma \times M$ and $\mathcal{R}_{\phi}$ is open and saturated in $\Sigma \times M$, but dense in $\partial \Sigma \times M$, such that the varying volume form $\omega_{\phi(z, \cdot)}^{n}$ extends to a continuous $(n, n)$ form on $\Sigma^{0} \times M$, where $\Sigma^{0}=(\Sigma \backslash \partial \Sigma)$.

Clearly, if $\phi$ is a partially smooth solution, then its regular set $\mathcal{R}_{\phi}$ consists of all points where the vertical volume form $\omega_{\phi(z, \cdot)}^{n}$ is positive in $\Sigma \times M$.

Theorem 1.3.2. Suppose that $\Sigma$ is a unit disk. For every smooth map $\phi_{0}: \partial \Sigma \rightarrow \mathcal{H}$, there exists a unique partially smooth solution to (1.1).

\footnotetext{
${ }^{7}$ This follows from sub-harmonic property of the K-energy with respect to almost smooth solution as shown in the subsequent computation.
} 
In particular, this theorem implies that the $C^{1,1}$ solution from 8 is smooth on some open subset of $\Sigma \times M$ which is also dense in $\partial \Sigma \times M$. We expect this density property holds in interior as well. Indeed, it is true for generic boundary values, moreover, we can estimate the size of the singular set $\mathcal{S}_{\phi}=\Sigma \times M \backslash \mathcal{R}_{\phi}$.

Definition 1.3.3. We say that a solution $\phi$ of (1.1) is almost smooth if

1. it is partially smooth,

2. The distribution $\mathcal{D}_{\phi}$ extends to a continuous distribution in a saturated set $\tilde{\mathcal{V}} \subset \Sigma \times M$, such that the complement $\tilde{\mathcal{S}}_{\phi}$ of $\tilde{\mathcal{V}}$ is locally extendable ${ }^{8}$ and $\phi$ is $C^{1}$ continuous on $\tilde{\mathcal{V}}$. The set $\tilde{\mathcal{S}}_{\phi}$ is referred as the singular set of $\phi$.

3. The leaf vector field $X$ is uniformly bounded in $\mathcal{D}_{\phi}$.

A smooth solution is certainly an almost smooth solution of (1.1). For a sequence of almost smooth solutions whose boundary values converge in certain smooth topology, then it converges to a partially smooth solution in $C^{1, \beta}$-topology $(0<\beta<1)$.

Theorem 1.3.4. Suppose that $\Sigma$ is a unit disk. For any $C^{k, \alpha} \operatorname{map} \phi_{0}: \partial \Sigma \rightarrow \mathcal{H}(k \geq 2$, $0<\alpha<1)$ and for any $\epsilon>0$, there exists a $\phi_{\epsilon}: \partial \Sigma \rightarrow \mathcal{H}$ in the $\epsilon$-neighborhood of $\phi_{0}$ in $C^{k, \alpha}(\Sigma \times M)$-norm, such that (1.1) has an almost smooth solution with boundary value $\phi_{\epsilon}$.

This partial regularity is sharp since we do have examples (at least implicitly) where the solution for (1.1) corresponds to a singular metric (cf. [1]).

Theorem 1.3.2 will be derived from Theorem 1.3.4 by using estimates developed in late sections. ${ }^{9}$ The importance of Theorem 1.3.4 lies in the following theorem, which implies that an almost smooth solution is as good as a smooth solution when one concerns the K-energy.

Theorem 1.3.5. Suppose that $\phi$ is a partially smooth solution to (1.1). For every point $z \in \Sigma$, let $\mathbf{E}(z)$ be the K-energy (or modified $K$ energy) evaluated on $\phi(z, \cdot) \in \overline{\mathcal{H}}$. Then $\mathbf{E}$ is a bounded subharmonic function on $\Sigma$ in the sense of distribution, moreover, we have the following

$$
\int_{\mathcal{R}_{\phi}}\left|\mathbf{D} \frac{\partial \phi}{\partial z}\right|_{\omega_{\phi(z, \cdot)}^{2}} \frac{\sqrt{-1}}{2} d z \wedge d \bar{z} \wedge \omega_{\phi(z, \cdot)}^{n} d z d \bar{z} \leq\left.\int_{\partial \Sigma} \frac{\partial \mathbf{E}}{\partial \mathbf{n}}\right|_{\partial \Sigma} d s
$$

where $d s$ is the length element of $\partial \Sigma$ and for any smooth function $\theta, \mathbf{D} \theta$ denotes the (2,0)-part of $\theta$ 's Hessian with respect to the metric $\omega_{\phi(z,)}$. The equality holds if $\phi$ is almost smooth.

\footnotetext{
${ }^{8} \mathrm{~A}$ closed subset $S \subset \Sigma \times M$ of measure 0 is locally extendable if for any continuous function in $\Sigma \times M$ which is $C^{1,1}$ on $\Sigma \times M \backslash S$ can be extended to a $C^{1,1}$ function on $\Sigma \times M$. Notice that any set of codimension 2 or higher is automatically locally extendable.

${ }^{9}$ It is believed that for any smooth boundary map $\phi_{0}: \partial \Sigma \mapsto \mathcal{H}$, the corresponding $C^{1,1}$ solution is almost smooth. It is also interesting to estimate precise size of $\mathcal{S}_{\phi}$.
} 
Theorem 1.1.2 follows from this theorem and the observation on subharmonic functions over an infinite strip at the beginning of this subsection.

Another corollary of this theorem is

Proposition 1.3.6. If there are two constant scalar curvature metrics (resp. two extremal Kähler metrics), there exists a path in $\overline{\mathcal{H}}$ of $C^{1,1}$-functions $\phi_{t}(0 \leq t \leq 1)$ which connects those two metrics, such that the K-energy (resp. modified $K$ energy) achieves its minimum at every $\phi_{t}$ along the path.

It was conjectured by the first named author that any $C^{1,1}$ function which attains the absolute minimum of the K-energy (or the modified K-energy) is actually smooth (cf. [8], 7]). In this paper, we will confirm this conjecture in the case that the function arises from Proposition 1.3.6 This is sufficient for completing the proof of Theorem 1.1.1 that is, the uniqueness theorem of extremal Kähler metrics in any given Kähler class. The conjecture will be proved by first establishing a partial $C^{1}$-regularity for the vertical volume form of any $C^{1,1} \mathrm{~K}$-energy minimizers.

The major part of this paper will be devoted to proving these main technical results as we described above. We believe that our techniques developed in this paper can be applied to studying regularity of a much wider class of degenerate complex Monge-Ampere equations.

\subsection{Ideas for Proof of Theorem 1.3.4}

It has been known for a long time that solutions of the homogeneous complex Monge-Ampere equation are closely related to foliations by holomorphic curves (cf. [19, [26], 11]). In [26], S. Semmes formulated the Dirichlet problem for (1.1) in terms of a foliation by holomorphic curves with boundary in a totally real submanifold of the complex cotangent bundle of the underlying manifold.

In [26] (also see [11]), Semmes constructed a complex manifold $\mathcal{W}_{[\omega]}$ with a holomorphic $(2,0)$-form $\Theta$ ) to each Kähler class $[\omega]$. There is a natural projection $\pi: \mathcal{W}_{[\omega]} \mapsto M$. He observed that for any $\phi \in \mathcal{H}$, we can associate a complex submanifold $\Lambda_{\phi}$ in $\mathcal{W}_{[\omega]}$ such that

$$
\left.\Theta\right|_{\Lambda_{\phi}}=-\sqrt{-1} \omega_{\phi}
$$

that is, $\left.\operatorname{Re}(\Theta)\right|_{\Lambda_{\phi}}=0$ and $-\left.\operatorname{Im}(\Theta)\right|_{\Lambda_{\phi}}=\omega_{\phi}>0$. Locally, $\Lambda_{\phi}$ is simply the graph of $\partial(\rho+\phi)$ where $\omega=\sqrt{-1} \partial \bar{\partial} \rho$. This means that $\Lambda_{\phi}$ is an exact Lagrangian symplectic submanifold of $\mathcal{W}_{[\omega]}$ with respect to $\Theta$. Conversely, given an exact Lagrangian symplectic submanifold $\Lambda$ of $\mathcal{W}_{[\omega]}$, one can construct a smooth function $\phi$ such that $\Lambda=\Lambda_{\phi}$. Hence, Kähler metrics in the Kähler class $[\omega]$ are in one-to-one correspondence with exact Lagrangian symplectic submanifolds of $\mathcal{W}_{[\omega]}$.

Given $\phi_{0}: \partial \Sigma \mapsto \mathcal{H}$, define

$$
\bar{\Lambda}_{\phi_{0}}=\left\{(\tau, v) \in \partial \Sigma \times \mathcal{W}_{[\omega]} \mid v \in \Lambda_{\phi_{0}(\tau)}\right\}
$$


One can show that $\bar{\Lambda}_{\phi_{0}}$ is a totally real sub-manifold in $\Sigma \times \mathcal{W}_{[\omega]}$. So it makes sense to study the moduli space of holomorphic disks with boundary in $\bar{\Lambda}_{\phi_{0}}$. Its significance is clear from the following result from [26] (also see [11]).

Proposition 1.4.1. Assume that $\Sigma$ is simply connected. For any boundary map $\phi_{0}: \partial \Sigma \rightarrow \mathcal{H}$, there is a solution $\phi \in C^{\infty}(\Sigma, \mathcal{H})$ of (1.1) with boundary value $\phi_{0}$ if and only if there is a smooth family of holomorphic maps $h_{x}: \Sigma \mapsto \mathcal{W}_{[\omega]}$ parametrized by $x \in M$ satisfying: (1) $\pi\left(h_{x}\left(z_{0}\right)\right)=x$, where $z_{0}$ is a given point in $\Sigma \backslash \partial \Sigma$; (2) $h_{x}(\tau) \in \Lambda_{\phi_{0}(\tau)}$ for each $\tau \in \partial \Sigma$ and $x \in M$; (3) For each $z \in \Sigma$, the map $\gamma_{z}(x)=\pi\left(h_{x}(z)\right)$ is a diffeomorphism of $M$.

In [11], S. Donaldson used this fact to study deformations of smooth solutions for (1.1) as the boundary value varies. This inspired us to study foliations by holomorphic disks in order to have a partial regularity theory for (1.1). Theorem 1.3.4 will be proved by establishing existence of foliations by holomorphic disks with relatively mild singularities. More precisely, we will show that for a generic boundary value, there is an open set in the moduli space of holomorphic disks which generates a foliation on $\Sigma \times M \backslash S$ for a closed subset $S$ of codimension at least one.

Now let us fix a generic boundary value $\phi_{0}$ and study the corresponding moduli $\mathcal{M}_{\phi_{0}}$ of holomorphic disks. First it follows from the Index theorem that the expected dimension of this moduli is $2 n$. Recall that a holomorphic disk $u$ is regular if the linearized $\bar{\partial}$-operator $\bar{\partial}_{u}$ has vanishing cokernel. The moduli space is smooth near a regular holomorphic disk. Following [11, we call $u$ super-regular if there is a basis $s_{1}, \cdots, s_{2 n}$ of the kernel of $\bar{\partial}_{u}$ such that $d \pi\left(s_{1}\right)(x), \cdots, d \pi\left(s_{2 n}\right)(x)$ span $T_{u(x)} M$ for every $x \in \Sigma$, where $\pi: \Sigma \times \mathcal{W}_{[\omega]} \mapsto \Sigma \times M$ is the natural projection. We call $u$ almost super-regular if $d \pi\left(s_{1}\right)(x), \cdots, d \pi\left(s_{2 n}\right)(x) \operatorname{span} T_{u(x)} M$ for every $x \in \Sigma \backslash \partial \Sigma$. Clearly, the set of super-regular disks is open.

One of our crucial observations is that Semmes' arguments can be made local along superregular holomorphic disks. First let us introduce the notion of a nearly smooth foliation.

Definition 1.4.2. A nearly smooth foliation $\mathcal{F}_{\phi_{0}}$ associated to a boundary value $\phi_{0}$ is given by an open subset $\mathcal{U}_{\phi_{0}} \subset \mathcal{M}_{\phi_{0}}$ of super-regular disks whose images in $\Sigma \times M$ give rise to a foliation on an open-dense set $\mathcal{V}_{\phi_{0}}$ of $\Sigma \times M$ such that

1. this foliation can be extended to be a continuous foliation by holomorphic disks in an open set $\tilde{\mathcal{V}}_{\phi_{0}} \subset(\Sigma \backslash \partial \Sigma) \times M$ such that it admits a continuous lifting in $\Sigma \times \mathcal{W}_{M}$;

2. the complement of $\tilde{\mathcal{V}}_{\phi_{0}}$ in $\Sigma \times M$ is locally extendable.

3. The leaf vector field (cf. definition below) induced by the foliation in $\mathcal{V}_{\phi_{0}}$ is uniformly bounded.

Definition 1.4.3. For each $(z, x) \in \mathcal{V}_{\phi_{0}}$, the leaves of $\mathcal{V}_{\phi_{0}}$ give rise to a vector field of the form

$$
\frac{\partial}{\partial z}+X, \quad \text { where } X \in T_{z, x}^{1,0} M .
$$

This $X$ is called the leaf vector field in $\mathcal{V}_{\phi_{0}}$. 
Proposition 1.4.1 has the following generalization.

Theorem 1.4.4. Almost smooth solutions of (1.1) are in one-to-one correspondence with nearly smooth foliations. Moreover, if $\phi_{0}$ is generic, the corresponding almost smooth solution $\phi$ has additional properties: $\omega_{\phi}$ is a smooth $(1,1)$ form in $\Sigma \times M \backslash \tilde{\mathcal{S}}_{\phi}$ and the singular set $\tilde{\mathcal{S}}_{\phi}$ has codimension at least 2 in each slice $\{z\} \times M, \forall z \in \Sigma^{0} .^{10}$

Thus, in order to prove Theorem 1.3.4 we only need to show the following

Theorem 1.4.5. For a generic boundary value $\phi_{0}$, there is a nearly smooth foliation associated to $\phi_{0}$ generated by an open set $\mathcal{U}_{\phi_{0}}$ of the corresponding moduli space $\mathcal{M}_{\phi_{0}}$. Moreover, the set of holomorphic disks which are neither super-regular nor almost super-regular has codimension at least two in the closure of $\mathcal{U}_{\phi_{0}}$ in $\mathcal{M}_{\phi_{0}}$.

The idea for proving Theorem 1.4.5 is outlined as follows. Let $\phi_{0}$ be a generic boundary value such that $\mathcal{M}_{\phi_{0}}$ is smooth. This follows from a result of Oh on transversality. By the same transversality argument, one can show that there is a generic path $\phi_{t}(0 \leq t \leq 1)$ such that $\phi_{1}=0$ and the total moduli $\tilde{\mathcal{M}}=\bigcup_{t \in[0,1]} \mathcal{M}_{\phi_{t}}$ is smooth. Moreover, we may assume that $\mathcal{M}_{\phi_{t}}$ are smooth for all $t$ except finitely many $t_{1}, \cdots, t_{N}$ where the moduli space may have isolated singularities. It follows from Semmes and Donaldson's work-Proposition 1.4.1 that $\mathcal{M}_{1}$ has at least one component which gives a foliation for $\Sigma \times M$. We want to show that this component will deform to a component of $\mathcal{M}_{\phi_{0}}$ which generates a nearly smooth foliation. We will use the continuity method. Assume that $\phi$ is the unique $C^{1,1}$-solution of (1.1) with boundary value $\phi_{t}$ for some $t \in[0,1]$. Let $f$ be any holomorphic disk in the component of $\mathcal{M}_{\phi_{t}}$ which generates the corresponding foliation.

Using the $C^{1,1}$ bound on $\phi$, one can have a uniform area ${ }^{11}$ bound on holomorphic disks in $\mathcal{M}_{\phi_{t}}$. It follows from an extension of Gromov's compactness theorem that any sequence of such holomorphic disks has a subsequence which converges to a holomorphic disk together with possibly finitely many bubbles. These bubbles which occur in the interior are holomorphic spheres, while bubbles in the boundary might be holomorphic spheres or disks. We will show that no bubbles can actually occur. According to E. Calabi and X. Chen [6], this infinite dimensional space $\mathcal{H}$ is non-positively curved in the sense of Alexanderov. Heuristically speaking, we can exploit this curvature condition to rule out the existence of interior bubbles. One can also rule out boundary bubbles by using the non-positivity and totally real property of the boundary condition. Since there are no bubbles, the Fredholm index of holomorphic disks is invariant under the limiting process. This is an important fact needed in our doing deformation theory.

In order to get a nearly smooth foliation, we need to prove that the moduli space has an open set of super-regular holomorphic disks for each $t$. First we observe that the set of super-

\footnotetext{
${ }^{10}$ The corresponding nearly smooth foliations have additional properties and will be called almost super-regular foliations (cf. Section 3.3).

${ }^{11}$ We actually calculate area of the image of disks in $\Sigma \times M$.
} 
regular disks is open. Moreover, using the transversality arguments, one can show that for a generic path $\phi_{t}$, the closure of all super-regular disks in each $\mathcal{M}_{\phi_{t}}$ is either empty or forms an irreducible component. This implies the openness. It remains to prove that each moduli has at least one super-regular disk. It is done by using capacity estimates and curvature estimate along super-regular holomorphic disks (cf. Sections 4 and 5 for details).

\subsection{Organization}

In Section 2, we establish the correspondence between homogeneous complex Monge-Ampere equations and foliations by holomorphic curves. The goal is to prove Theorem 1.4.4. The proof is based on a local version of Semmes' construction. Semmes's construction is global in nature and was re-diskovered in Donaldson's work 11. In Section 3, we show necessary transversality results. In particular, we show that the set of boundary values such that corresponding moduli space $\mathcal{M}$ induces an almost super regular foliation is generically open. In Section 4, we study the defomation of holomorphic disks arising from a smooth solution to a homogenous complex Monge-Ampere equation. This is a local theory which is used in Section 2,3 and late sections as well. In Section 5, we prove the set of boundary values such that corresponding moduli space $\mathcal{M}$ induces an almost super regular foliation is closed. This will be done by proving a volume ratio estimate via a capacity argument. In Section 5, we prove that the $\mathrm{K}$ energy function is subharmonic when restricted to a disk family of almost smooth solutions, which in turns implies that the $\mathrm{K}$ energy function is always bounded from below. In section 7 , we derive a partial $C^{1}$ regularity for the vertical volume form of any $C^{1,1} \mathrm{~K}$-energy minimizer. We need to introduce a notion of weak Kähler Ricci flow to derive this a prioir estimate. In Section 8, we prove the uniqueness result for extremal Kähler metrics. In the Appendix, we discuss the Loop groups of $G L(n, C)$ and their relation to holomorphic discs. This is essentially written by Professor E. Lupercio.

Acknowledgement The first author is grateful to S. K. Donaldson for insightful discussions and constant encouragements during this project and over years, in particular, the first author is deeply indebted to him during the period of time when both of us were visiting Stanford University in 1998.

\section{Foliations and the Homogenous comlex Monge Ampere Equa- tion}

In this section, we diskuss the correspondence between homogeneous complex Monge-Ampere equations and foliations by holomorphic disks. We will prove Theorem 1.4.4. 


\subsection{Semmes' construction}

In [26], Semmes associated a complex manifold $\mathcal{W}_{[\omega]}$ to each Kähler class $[\omega]:$ Let $\left\{U_{i}, i \in \mathcal{I}\right\}$ be a covering of $M$ such that $\left.\omega\right|_{U_{i}}=\sqrt{-1} \partial \bar{\partial} \rho_{i}$, where $\mathcal{I}$ is an index set. For any $x=y \in$ $U_{i} \cap U_{j}(i, j \in \mathcal{I})$, we identify $\left(x, v_{i}\right) \in T^{*} U_{i}$ with $\left(y, v_{j}\right) \in T^{*} U_{j}$ if $v_{i}=v_{j}+\partial\left(\rho_{i}-\rho_{j}\right)$. Then $\mathcal{W}_{[\omega]}$ consists of all these equivalence classes of $\left[x, v_{i}\right]$. There is a natural map $\pi: \mathcal{W}_{[\omega]} \mapsto T^{*} M$, assigning $\left(x, v_{i}\right) \in T^{*} U_{i}$ to $\left(x, v_{i}-\partial \rho_{i}\right)$. Then the complex structure on $T^{*} M$ pulls back to a complex structure on $\mathcal{W}_{[\omega]}$, moreover, there is also a canonical holomorphic 2-form $\Theta$ on $\mathcal{W}_{[\omega]}$, in terms of canonical local coordinates $z_{\alpha}, \xi_{\alpha}(\alpha=1, \cdots, n)$ of $T^{*} U_{i}$,

$$
\Theta=d z_{\alpha} \wedge d \xi_{\alpha}
$$

Now for any $\phi \in \mathcal{H}$, we can associate a complex submanifold $\Lambda_{\phi}$ in $\mathcal{W}_{[\omega]}$ : For any open subset $U$ on which $\omega$ can be written as $\sqrt{-1} \partial \bar{\partial} \rho$, we define $\left.\Lambda_{\phi}\right|_{U} \subset \mathcal{W}_{[\omega]}$ to be the graph of $\partial(\rho+\phi)$ in $T^{*} U$. Clearly, this $\Lambda_{\phi}$ is independent of the choice of $U$. A straightforward computation shows

$$
\left.\Theta\right|_{\Lambda_{\phi}}=-\sqrt{-1} \omega_{\phi}
$$

that is, $\left.\operatorname{Re}(\Theta)\right|_{\Lambda_{\phi}}=0$ and $-\left.\operatorname{Im}(\Theta)\right|_{\Lambda_{\phi}}=\omega_{\phi}>0$. This means that $\Lambda_{\phi}$ is an exact Lagrangian symplectic submanifold of $\mathcal{W}_{[\omega]}$ with respect to $\Theta$. Conversely, given an exact Lagrangian symplectic submanifold $\Lambda$ of $\mathcal{W}_{[\omega]}$, we have a smooth function $\phi$ such that $\Lambda=\Lambda_{\phi}$. Hence, Kähler metrics with Kähler class $[\omega]$ are in one-to-one correspondence with exact Lagrangian symplectic submanifolds in $\mathcal{W}_{[\omega]}$.

This was briefly diskussed in our introduction. We refer the readers to both [26] and [11] for more details. For the readers' convenience, let us briefly explain the proof of Proposition 1.4.1. Let $\phi$ be a solution of (1.1) on $\Sigma \times M$ such that $\phi(z, \cdot) \in \mathcal{H}$ for any $z \in \Sigma$. Recall that there is an induced distribution $\mathcal{D}_{\phi} \subset T(\Sigma \times M)$ by

$$
\left.\mathcal{D}_{\phi}\right|_{p}=\left\{v \in T_{p}(\Sigma \times M) \mid i_{v}\left(\pi_{2}^{*} \omega+\sqrt{-1} \partial \bar{\partial} \phi\right)=0\right\}, \quad p \in \Sigma \times M .
$$

It is a holomorphic integrable distribution. If $\Sigma$ is simply-connected and $\phi(z, \cdot) \in \mathcal{H}$ for each $z \in \Sigma$, then the leaf of $\mathcal{D}_{\phi}$ containing $\left(z_{0}, x\right)$ is the graph of a holomorphic map $f_{x}: \Sigma \mapsto M$ with $f_{x}\left(z_{0}\right)=x$. If we write $f_{x}(z)=\sigma_{z}(x)$ we get a family of diffeomorphisms $\sigma_{z}$ of $M$ with $\sigma_{z_{0}}=\operatorname{Id}_{M}$. Now for any fixed $z$ we have a Kähler form $\omega+\sqrt{-1} \partial \bar{\partial} \phi(z, \cdot)$ on $M$ and hence a section $s_{z}: M \mapsto \mathcal{W}_{[\omega]}$ whose image is the exact Lagrangian symplectic graph $\Lambda_{\phi(z, \cdot)}$. Then $h_{x}(z)=\gamma_{z}(x)=s_{z}\left(f_{x}(z)\right)$ as required. This process can be reversed. Since we have to carry out this reversed process in the proof of Theorem 1.4.4, we omit details here and refer the readers to the next subsection.

\subsection{Local Uniqueness for Equation (1.1)}

We will prove uniqueness of compatible solutions for (1.1) near a super-regular disk. We will define a compatible solution as follows. 
Given a boundary value $\phi_{0}$ on $\partial \Sigma \times M$. Suppose that $\mathcal{F}_{\phi_{0}}$ is a nearly smooth foliation (cf. Definition 1.4.2). A solution $\phi$ of (1.1) with boundary value $\phi_{0}$ in an open subset $\mathcal{V} \subset \Sigma \times M$ is called compatible with this foliation $\mathcal{F}_{\phi_{0}}$ if

1. $\mathcal{V}$ is saturated with respect to $\mathcal{F}_{\phi_{0}}$;

2. $\omega+\sqrt{-1} \partial \bar{\partial} \phi(z, \cdot)$ is a family of Kähler metrics on $M$;

3. The kernel of $\pi_{2}^{*} \omega+\sqrt{-1} \partial \bar{\partial} \phi$ lies in the distribution induced by $\mathcal{F}_{\phi_{0}}$.

Theorem 2.2.1. Two compatible solutions of (1.1) with respect to the nearly smooth foliation $\mathcal{F}_{\phi_{0}}$ coincides along the intersection of their domains.

We will adopt the notations from previous sections. First we recall the integrable distribution

$$
\left.\mathcal{D}_{\phi}\right|_{(z, x)}=\left\{v \in T_{z} \Sigma \times T_{x} M \mid i_{v}\left(\pi_{2}^{*} \omega+\sqrt{-1} \partial \bar{\partial} \phi\right)=0\right\}, \quad(z, x) \in \mathcal{R}_{\phi} .
$$

Here $i_{v}$ denotes the interior product. Since $\mathcal{R}_{\phi}$ is saturated, every maximal integral submanifold of $\mathcal{D}_{\phi}$ in $\mathcal{R}_{\phi}$ is a disk and closed in $\Sigma \times M$.

Lemma 2.2.2. For any $f \in \mathcal{U}_{\phi_{0}}$, suppose that $\mathcal{O}_{f}$ is a saturated open neighborhood of the image of $f$ in $\Sigma \times M$. Suppose that $\phi_{f}$ is a solution of (1.1) on $\mathcal{O}_{f}$ compatible with $\mathcal{F}_{\phi_{0}}$. Then, for any $\tilde{f}: \Sigma \mapsto \mathcal{V}_{\phi_{0}}$ such that the image of $\pi \cdot \tilde{f}$ lies completely in $\mathcal{O}_{f}$, we have

$$
\frac{\partial^{2}}{\partial z \partial \bar{z}}\left(\phi_{f}(\pi \circ \tilde{f}(z))\right)=-|\partial(\pi \circ \tilde{f})|_{\omega}^{2}(\pi \circ \tilde{f}(z)), \quad \forall z \in \Sigma,
$$

and

$$
\phi_{f}(\pi \circ \tilde{f}(z))=\phi_{0}(\pi \circ \tilde{f}(z)), \quad \forall z \in \partial \Sigma .
$$

This lemma implies that $\phi_{f}$ is uniquely determined by only the geometric conditions along the image of each leaf. Theorem 2.2.1 follows from this lemma.

Using this theorem, we can patch together $\phi_{f}$, initially defined on small open, saturated sets around a super regular leaf, to obtain a smooth potential function $\phi$ in $\mathcal{U}_{\phi_{0}}$. This function $\phi$ solves (1.1) and satisfies (2.4) - (2.5).

\subsection{Almost Smooth Solutions $\Leftrightarrow$ Nearly Smooth Foliations}

In this subsection, we establish the equivalence between almost smooth solutions of (1.1) and nearly smooth foliations. This generalizes Semmes' construction. We will adopt notations from previous subsections.

Proposition 2.3.1. An almost smooth solution to the equation (1.1) with boundary value $\phi_{0}$ induces a nearly smooth foliation associated to $\phi_{0}$. 
Proof. Let $\phi$ be an almost smooth solution with boundary value $\phi_{0}: \partial \Sigma \mapsto \mathcal{H}$. For every point $(z, x) \in \mathcal{R}_{\phi}$, there is a unique holomorphic map $f \in \mathcal{M}_{\phi_{0}}$ whose corresponding map $\pi \circ f: \Sigma \mapsto \Sigma \times M$ passes through $(z, x)$. The property that $\mathcal{R}_{\phi}$ is saturated implies that $\pi \circ f$ is a holomorphic disk and extends to the boundary of $\Sigma \times M$. According to Donaldson [11, such a holomorphic disk is super-regular. All these super-regular disks from $\mathcal{R}_{\phi_{0}}$ give rise to this open set $\mathcal{U}_{\phi_{0}} \subset \mathcal{M}_{\phi_{0}}$. The other two conditions of a nearly smooth foliation can be verified in a straightforward fashion. In other words, an almost smooth solution indeed induces a nearly smooth foliation $\mathcal{F}_{\phi_{0}}$.

Theorem 1.4.4 follows from the above proposition and the following.

Theorem 2.3.2. If $\mathcal{F}_{\phi_{0}}$ is a nearly smooth foliation (cf. Definition 1.4.2) associated to a boundary value $\phi_{0}: \partial \Sigma \rightarrow \mathcal{H}$, then there is an almost smooth solution $\phi$ to (1.1) with boundary value $\phi_{0}$.

The rest of this subsection is devoted to proving this theorem. Let $\mathcal{U}_{\phi_{0}}$ and $\mathcal{V}_{\phi_{0}}$ be the open subsets of $\mathcal{M}_{\phi_{0}}$ and $\Sigma \times M$ as given by $\mathcal{F}_{\phi_{0}}$. By definition, the induced foliation in $\mathcal{V}_{\phi_{0}}$ can be extended to be a continuous foliation by holomorphic disks in an open and dense subset $\tilde{\mathcal{V}}_{\phi_{0}}$ such that it admits a continuous lifting to $\Sigma \times \mathcal{W}_{M}$. Moreover, $\Sigma \times M \backslash \tilde{\mathcal{V}}_{\phi_{0}}$ is locally extendable.

Proposition 2.3.3. There is a smooth family of non-degenerate, closed $(1,1)$ forms $\tilde{\omega}(z, \cdot)$ defined on $\mathcal{V}_{\phi_{0}} \cap\{z\} \times M$ and a closed $(1,1)$ form $\Omega$ in $\mathcal{V}_{\phi_{0}}$ such that

1. $\tilde{\omega}=\omega_{\phi_{0}}$ in $\partial \Sigma \times M$, wherever $\tilde{\omega}$ is defined;

2. The restriction of $\tilde{\omega}$ to each leaf is a constant form;

3. $\Omega$ is defined by the following conditions:

$$
\left.\Omega\right|_{\{z\} \times M}=\tilde{\omega}, \quad \text { and } \quad i_{\frac{\partial}{\partial z}+X} \Omega=0,
$$

where $X$ is the leaf vector field in $\mathcal{V}_{\phi_{0}}$ induced by the nearly smooth foliation (cf. Defi. 1.4.3).

Proof. This is a local theorem. The proof can be found in [26] [11].

Next we want to show that $\Omega=\pi_{2}^{*} \omega_{0}+i \bar{\partial} \partial \phi$ for a compatible function $\phi$ in $\Sigma \times M$. We want to define this potential function in a small open and saturated neighborhood of any super regular leaf. Theorem 2.2.1 implies that two different locally defined compatible solutions of (1.1) must agree with each other on the overlap of their domains of definition. Since $\mathcal{V}_{\phi_{0}}$ is dense in $\Sigma \times M$, this defines $\phi$ in $\Sigma \times M$. The final step is to show that $\phi$ is uniformly $C^{1,1}$ and solve (1.1). Note that this approach is different from [11] since we are not dealing with a super regular foliation. 
Proposition 2.3.4. For any super regular leaf $f$, there exists a smooth function $\phi$ defined in a small tubular neighborhood $\mathcal{O}_{f}$ (which is saturated with respect to $\mathcal{F}_{\phi_{0}}$ ) of $\pi \circ \operatorname{ev}(f) \subset \Sigma \times M$ such that

$$
\begin{aligned}
\Omega=\pi_{2}^{*} \omega_{0}+i \bar{\partial} \partial \phi, & & \text { on } \mathcal{O}_{f} \subset \Sigma \times M \\
\phi=\phi_{0}, & & \text { on } \mathcal{O}_{f} \bigcap(\partial \Sigma \times M) .
\end{aligned}
$$

Remark 2.3.5. The potential function $\phi$ can be also defined in a tubular neighborhood $\mathcal{O}_{f}$ of an almost super regular leaf $f$. The function is smooth in $\mathcal{O}_{f} \bigcap\left(\Sigma^{0} \times M\right)$.

Proof. Recall that $M=\bigcup_{i \in \mathcal{I}} U_{i}$ and $\rho_{i}(i \in \mathcal{I})$ is the local defining potential function for $\omega_{0}$. For any point $(z, x) \in \mathcal{V}_{\phi_{0}}$, suppose that $x \in U_{i}$ for some $i \in \mathcal{I}$. In local coordinates, write

$$
\omega_{0}=\sum_{\alpha, \beta=1}^{n} \frac{\partial^{2} \rho_{i}}{\partial w_{\alpha} \partial w_{\bar{\beta}}} d w^{\alpha} \wedge d w^{\bar{\beta}}, \quad \tilde{\omega}=\sum_{\alpha, \beta=1}^{n} \frac{\partial^{2}\left(\rho_{i}+\phi\right)}{\partial w_{\alpha} \partial \bar{w}_{\beta}} d w^{\alpha} \wedge d w^{\bar{\beta}} .
$$

We can express the image of $\Sigma$ as ${ }^{12}$

$$
\{(z, x=f(z), \xi(x)), \forall z \in \Sigma\},
$$

where

$$
\xi(x)=\partial\left(\phi+\rho_{i}\right) .
$$

Since $\xi(x)$ is uniquely detrmined by image of an open set of super regular disks in $\Sigma \times \mathcal{W}_{M}$, then $\phi$ is uniquely determined by $\xi$, or by the structure of $\mathcal{W}_{M}$, up to a constant in $U_{i} \subset M$. In particular, in $(\partial \Sigma \times M) \cap \mathcal{V}_{\phi_{0}}$, we have $\phi=\phi_{0}$ modular some function in $z$ locally.

By definition, we write

$$
\begin{aligned}
\Omega=\omega_{0} & +\sum_{\substack{\alpha, \beta=1 \\
\text { n }}}^{n} \frac{\partial^{2} \phi}{\partial w_{\alpha} \partial \bar{w}_{\beta}} d w_{\alpha} d \bar{w}_{\beta}+\sum_{\alpha=1}^{n} \zeta^{\alpha} d w_{\alpha} d \bar{z} \\
& +\sum_{\beta=1}^{n} \zeta^{\bar{\beta}} d z d w_{\bar{\beta}}+h_{f} d z d \bar{z} .
\end{aligned}
$$

Since

$$
i_{\frac{\partial}{\partial z}+X} \Omega=0 \text { and } X=\sum_{1}^{n} \eta^{\alpha} \frac{\partial}{\partial w^{\alpha}}
$$

we have

$$
\zeta^{\alpha}+\left(g_{0, \alpha \bar{\beta}}+\frac{\partial^{2} \phi}{\partial w^{\alpha} \partial w^{\bar{\beta}}}\right) \eta^{\bar{\beta}}=0, \quad \forall \alpha=1,2, \cdots n .
$$

\footnotetext{
${ }^{12}$ Here $x=\pi_{2} \circ \pi \circ f(z)$ in the formula. However, for notation simpliticity, we simplify it as $x=f(z)$. This convention will be used later. This should cause no confusion.
} 
On the other hand, since $\xi(f(z))$ is a holomorphic function of $z$, we have

$$
\begin{aligned}
0 & =\frac{\partial \xi^{\alpha}}{\partial \bar{z}} \\
& =\frac{\partial^{2} \phi}{\partial w^{\alpha} \partial \bar{z}}+\frac{\partial^{2}\left(\phi+\rho_{i}\right)}{\partial w^{\alpha} \partial w^{\beta}} \frac{\partial f^{\bar{\beta}}}{\partial \bar{z}} \\
& =\frac{\partial^{2} \phi}{\partial w^{\alpha} \partial \bar{z}}+\left(g_{0, \alpha \bar{\beta}}+\frac{\partial^{2} \phi}{\partial w^{\alpha} \partial w^{\beta}}\right) \eta^{\bar{\beta}}
\end{aligned}
$$

Then

$$
\zeta^{\alpha}=\frac{\partial^{2}\left(\phi+\rho_{i}\right)}{\partial w^{\alpha} \partial \bar{z}}, \quad \forall \alpha=1,2, \cdots n .
$$

Consequently, $\Omega$ takes the form:

$$
\begin{aligned}
\omega_{0}+\sum_{\alpha, \beta=1}^{n} & \frac{\partial^{2} \phi}{\partial w_{\alpha} \partial \bar{w}_{\beta}} d w_{\alpha} d \bar{w}_{\beta}+\sum_{\alpha=1}^{n} \frac{\partial^{2} \phi}{\partial \bar{z} \partial w_{\alpha}} d w_{\alpha} d \bar{z} \\
& +\sum_{\beta=1}^{n} \frac{\partial^{2} \phi}{\partial z \partial w_{\bar{\beta}}} d z d w_{\bar{\beta}}+h_{f} d z d \bar{z} .
\end{aligned}
$$

Moreover, the following hold about the leaf vector $X$ :

$$
\frac{\partial^{2} \phi}{\partial w^{\alpha} \partial \bar{z}}=-\left(g_{0, \alpha \bar{\beta}}+\frac{\partial^{2} \phi}{\partial w^{\alpha} \partial w^{\bar{\beta}}}\right) \eta^{\bar{\beta}}, \quad \forall \alpha=1,2, \cdots n .
$$

Using this equation and the fact that $\Omega^{n+1}=0$, we have

$$
\begin{aligned}
0 & =\left(h_{f}-g_{\varphi}{ }^{\alpha \bar{\beta}} \cdot \frac{\partial^{2} \phi}{\partial w^{\alpha} \partial \bar{z}} \cdot \frac{\partial^{2} \phi}{\partial w^{\beta} \partial z}\right) \cdot \tilde{\omega}^{n} \\
& =\left(h_{f}-\left(g_{0, \alpha \bar{\beta}}+\frac{\partial^{2} \phi}{\partial w^{\alpha} \partial w^{\beta}}\right) \eta^{\bar{\beta}} \eta^{\alpha}\right) \cdot \tilde{\omega}^{n} .
\end{aligned}
$$

Since $\tilde{\omega}$ is non-degenerate in $\mathcal{O}_{f}$, then

$$
h_{f}=\left(g_{0, \alpha \bar{\beta}}+\frac{\partial^{2} \phi}{\partial w^{\alpha} \partial w^{\bar{\beta}}}\right) \eta^{\bar{\beta}} \eta^{\alpha}
$$

is uniquely determined as well.

However (note $z=w_{n+1}$ ),

$$
\Omega-\sum_{\alpha, \beta=1}^{n+1} \sqrt{-1} \frac{\partial^{2}\left(\rho_{i}+\phi\right)}{\partial w_{\alpha} \partial w_{\bar{\beta}}} d w_{\alpha} d w_{\bar{\beta}}
$$

is a closed form. Consequently,

$$
l_{f} d z d \bar{z}=\left(h_{f}-\frac{\partial^{2} \phi}{\partial z \partial \bar{z}}\right) d z d \bar{z}
$$


is a closed form on $\mathcal{O}_{f}$. Therefore, $l_{f}$ is a function of $z$ only. Locally, we can replace $\phi$ by $\phi+K_{f}(z)$ where

$$
\frac{\partial^{2} K_{f}}{\partial z \partial \bar{z}}=-l_{f}
$$

After such a replacement, in each local coordinate chart in $\mathcal{O}_{f}$, we can choose the potential function $\phi$ uniquely, up to a harmonic function on $z$ only. This follows from the fact that

$$
\frac{\partial^{2} \phi}{\partial z \partial \bar{z}}=h_{f}
$$

where $h_{f}$ is uniquely determined by geometric data of super regular disks in $\mathcal{O}_{f}$.

Because of the unique extension property of harmonic functions, $\phi$ is uniquely determined in $\mathcal{O}_{f}$ by a global harmonic function in the $z$ direction. Choose such a potential function in $\mathcal{O}_{f}$ now. Notice that in $(\partial \Sigma \times M) \bigcap \mathcal{O}_{f}$, we have $\left.\omega_{z}\right|_{\partial \Sigma}=\omega_{\phi_{0}}$. Then, we can set

$$
\phi(z, \cdot)=\phi_{0}(z, \cdot)+m_{f}(z), \quad \forall z \in \partial \Sigma
$$

where $m_{f}(z)$ is a function of $z$ in $\Sigma$. Note that $m_{f}$ may not be harmonic function.

Choose a function $K_{f f}$ as a function of $z$ only such that

$$
\frac{\partial^{2} K_{f f}}{\partial z \partial \bar{z}}=0
$$

and

$$
\left.K_{f f}\right|_{\partial \Sigma}=m_{f} .
$$

This Drichelet problem has a unique solution. Now replace $\phi$ by $\phi-K_{f}$. Then $\Omega$ can be rewritten as $\pi_{2}^{*} \omega_{0}+\sqrt{-1} \partial \partial \phi$ in a tubular neighborhood of $\pi \circ f(\Sigma)$ in $\Sigma \times M$ such that $\phi=\phi_{0}$ in $(\partial \Sigma \times M) \cap \mathcal{O}_{f}$.

Now $\phi$ satisfies equation (1.1) on $\mathcal{V}_{\phi_{0}}$. Now we wish to extend it to solve the same equation in $\Sigma \times M$. The key step is to prove a global $C^{1,1}$ bound for $\phi$ in $\mathcal{V}_{\phi_{0}}$.

Proposition 2.3.6. We have $\omega_{\phi}>0$ when restricted to $M$ in $\mathcal{V}_{\phi_{0}}$.

Proof. For any point $\left(z, x^{\prime}\right) \in \mathcal{V}_{\phi_{0}}$, there exists a holomorphic leaf $f \in \mathcal{U}_{\phi_{0}}$ such that $\pi \circ e v(z, f)=$ $\left(z, x^{\prime}\right)$. For any $z \in \partial \Sigma$,

$$
\omega_{\phi}=\omega_{0}+\sqrt{-1} \partial \bar{\partial} \phi_{0}(z, \cdot)>0 .
$$

In other words, $\left.\omega_{\phi}\right|_{\pi \circ e v(\partial \Sigma, f)}>0$. However, $\left.\omega_{\phi}\right|_{\pi \circ e v(\Sigma, f)}$ is a constant form. Thus, $\omega_{\phi}$ is strictly positive for any $\left(z, x^{\prime}\right) \in \mathcal{V}_{\phi_{0}}$. 
It follows from this proposition that $\omega_{\phi}$ defines a smooth Kähler metric in $\mathcal{V}_{\phi_{0}}$. We want to show that this metric has a uniform $L^{\infty}$ bound in $\mathcal{V}_{\phi_{0}}$. In a local coordinate chart, write

$$
\left\{\begin{array}{l}
\omega_{0}=\sum_{\alpha, \beta=1}^{n} g_{0, \alpha \bar{\beta}} d w^{\alpha} d w^{\bar{\beta}}, \quad \omega_{\phi}=\sum_{\alpha, \beta=1}^{n} g_{\phi, \alpha \bar{\beta}} d w^{\alpha} d w^{\bar{\beta}}, \\
g_{\phi, \alpha \bar{\beta}}=g_{0, \alpha \bar{\beta}}+\frac{\partial^{2} \phi}{\partial w^{\alpha} \partial w^{\beta}}, \forall \alpha, \beta=1,2 \cdots n .
\end{array}\right.
$$

For any super regular leaf $f$ and for any $z \in \Sigma$, the restricted bundle $T_{f(z)} M$ at $f(z)$ is a trivial holomorphic bundle over $\Sigma$ with complex rank $n$. Restriction of $g_{\phi}$ to this $T M$ bundle induces an hermitian metric on this bundle. Denote by $F_{\beta}^{\alpha}(1 \leq \alpha, \beta \leq n)$ the curvature of this hermitian metric. We have the following formula (cf. Section 4),

$$
\begin{aligned}
F_{\alpha}^{r} & =-\partial_{\bar{z}}\left(g_{\phi}^{r \bar{\delta}} \partial_{z} g_{\phi, \alpha \bar{\delta}}\right) \\
& =-\frac{\partial \eta^{r}}{\partial w_{\bar{i}}} \cdot \frac{\partial \eta^{\bar{i}}}{\partial w_{\alpha}} \leq 0 .
\end{aligned}
$$

In particular, the curvature is always semi-negative. Now we are ready to state the maximum principle.

Proposition 2.3.7. (Maximum Principle along leaves) The Kähler metric $g_{\phi}$ is uniformly bounded from above in each leaf in $\mathcal{U}_{\phi_{0}}$.

Proof. First give a finite covering of $M=\bigcup_{i \in \mathcal{I}} U_{i}$. . There exists a uniform constant $C\left(g_{0}\right)$ which depends only on this covering such that in each of these coordinate chart, we have

$$
\left\|\frac{\partial g_{0, i \bar{j}}}{\partial w_{k}}\right\|_{g_{0}} \leq C\left(g_{0}\right), \quad \forall i, j, k=1,2, \cdots n .
$$

For any $f \in \mathcal{U}_{\phi_{0}}$, the restricted $T M$ bundle on $\pi \circ f(\Sigma)$ is a trivial holomorphic vector bundle over $\Sigma$. For any $(z, p)$ in this leaf, pick any $n$-frame $s_{1}, s_{2}, \cdots, s_{n} \in T_{p}^{1,0} M$ and extend these vectors over the disk as a frame of $n$ constant holomorphic sections in this $T^{1,0} M$ bundle. We still denote as $\left\{s_{1}, s_{2}, \cdots, s_{n}\right\}$. Note that in any coordinate charte $U_{i}$ chosen above, we have

$$
\frac{\partial}{\partial z} g_{0}(s, s)=\frac{\partial}{\partial z} g_{0, i \bar{j}} s^{i} s^{\bar{j}}=\frac{\partial g_{0, i \bar{j}}}{\partial w_{k}} X^{k} s^{i} s^{\bar{j}} \leq C\left(g_{0}\right) \max |X| \cdot g_{0}(s, s) .
$$

In other words, for these constant holomorphic section of the $T M$ bundle over $f(\Sigma)$, it is easy to see that

$$
\inf _{z \in \Sigma} g_{0}(s, s) \geq c_{0}(f) \max _{z \in \Sigma} g_{0}(s, s)
$$

where $c_{0}(f)$ depends on $g_{0}$ and the embedding of $f$ only. 
To prove the maximum principle for metric $g_{\phi}$ along the leaf, we just need to show that $g_{\phi}(s, s)(1 \leq \alpha \leq n)$ has a uniform upper bound for any constant holomorphic leaf the tangent bundle. If the upper bound is achieved on the boundary, then the claim is proved since $g_{\phi}=g_{\phi_{0}}$ in $\partial \Sigma \times M$. If the maximum is attained at some interior point $(z, p) \in \Sigma^{0} \times M$, choose an appropriate coordinate in $T_{p} M$. We may assume

$$
g_{\phi, \alpha \bar{\beta}}(z, p)=\delta_{\alpha \beta}(\forall \alpha, \beta=1,2, \cdots n),\left.\quad \partial_{z} g_{\phi}\right|_{(z, p)}=\left.\bar{\partial}_{z} g_{\phi}\right|_{(z, p)}=0 .
$$

At this point $(z, p)$, we have

$$
\begin{aligned}
\bar{\partial}_{z} \partial_{z} g_{\phi}(s(c), s(c)) & =g_{\phi}\left(\partial_{z} s(c), \partial_{z} s(c)\right)+\left(\bar{\partial}_{z} \partial_{z} g_{\phi}\right)(s(c), s(c)) \\
& =g_{\phi}\left(\partial_{z} s(c), \partial_{z} s(c)\right)-F(s(c), s(c)) \geq 0 .
\end{aligned}
$$

This shows that the maximum must achieve in the boundary. This along with inequality 2.10 gives desired upper bound on $g_{\varphi}$.

Using this proposition, we can prove

Theorem 2.3.8. There exists a uniform upper bound for the Kähler metric $g_{\phi}$ in $\mathcal{V}_{\phi_{0}}$.

Proof. This follows from the proof of the previous theorem as well as the fact that any sequence of holomorphic discs in $\mathcal{V}_{\phi_{0}}$ always have a subsequence which converges to an embedded holomorphic discs in $\Sigma \times M$.

Finally, we have

Theorem 2.3.9. $\phi$ is uniformly $C^{1,1}$ in $\Sigma \times M$, smooth in $\mathcal{V}_{\phi_{0}}$ such that it solves

$$
\left(\pi_{2}^{*} \omega_{0}+\sqrt{-1} \partial \bar{\partial} \phi\right)^{n+1}=0, \quad \text { in } \quad \Sigma \times M .
$$

Moreover, this solution coincides with the solution established in [8].

Proof. By Definition 1.4.2 $\phi$ can be extended to be a $C^{1}$ continuous function in $\Sigma \times M \backslash \tilde{\mathcal{S}}_{\phi_{0}}$. Since $\tilde{\mathcal{S}}_{\phi}$ is locally extendable and $\phi$ is uniformly $C^{1,1}$ bounded in $\mathcal{V}_{\phi_{0}}$, we can show that $\phi$ can be extended as a global $C^{1,1}$ function in $\Sigma \times M$. It follows that there is a sequence of Kähler potential $\left\{\phi_{m} \in \mathcal{H}, m \in \mathbb{N}\right\}$ such that $\phi_{m} \rightarrow \phi$ in weak $C^{1,1}(\Sigma \times M)$ topology. Moreover, the convergence is smooth in any compact subset of $\mathcal{V}_{\phi_{0}}$. It follows that for any smooth test function $\psi$, we have

$$
\lim _{m \rightarrow \infty} \int_{\Sigma \times M} \psi \omega_{\phi_{m}}^{n+1}=0 .
$$

Suppose $\varphi$ is the $C^{1,1}$ solution given by the first author in $[8$, we have 


$$
\begin{aligned}
0 & =\lim _{m \rightarrow \infty} \int_{\Sigma \times M}(\varphi-\phi)\left(\omega_{\varphi}^{n+1}-\omega_{\phi_{m}}^{n+1}\right) \\
& =\lim _{m \rightarrow \infty} \int_{\Sigma \times M}(\varphi-\phi)\left(\omega_{\varphi}-\omega_{\phi_{m}}\right)\left(\sum_{i=0}^{n} \omega_{\varphi}^{i} \wedge \omega_{\phi_{m}}^{n-i}\right) \\
& =-\lim _{m \rightarrow \infty} \int_{\Sigma \times M} \sqrt{-1} \partial(\varphi-\phi) \wedge \bar{\partial}\left(\varphi-\phi_{m}\right)\left(\sum_{i=0}^{n} \omega_{\varphi}^{i} \wedge \omega_{\phi_{m}}^{n-i}\right) \\
& =-\int_{\mathcal{V}_{\phi_{0}}} \sqrt{-1} \partial(\varphi-\phi) \wedge \bar{\partial}(\varphi-\phi)\left(\sum_{i=0}^{n} \omega_{\varphi}^{i} \wedge \omega_{\phi}^{n-i}\right) .
\end{aligned}
$$

It follows that $\phi=\varphi$ in $\mathcal{V}_{\phi_{0}}$ since $\omega_{\phi}$ is smooth in $\mathcal{V}_{\phi_{0}}$. Since $\mathcal{V}_{\phi_{0}}$ is dense in $\Sigma \times M$, it follows that the solution we constructed conincide with solution established in the first author's paper 8 .

\section{Deformation of holomorphic disks with totally real boundary}

\subsection{Local analysis of holomorphic disks}

For any boundary map $\phi_{0}: \partial \Sigma \rightarrow \mathcal{H}$, there is a $2 n+1$-dimensional totally real submanifold $\bar{\Lambda}_{\phi_{0}}=\bigcup_{z \in \partial \Sigma}\left(\{z\} \times \Lambda_{\phi_{0}(z, \cdot)}\right)$ in $\Sigma \times \mathcal{W}_{M}$. Consider the moduli space $\mathcal{M}_{\phi_{0}}$ of all of holomorphic disks $\rho:(\Sigma, \partial \Sigma) \rightarrow\left(\Sigma \times \mathcal{W}_{M}, \bar{\Lambda}_{\phi_{0}}\right)$ with vanishing normal Maslov index. Note that the normal bundle over $\rho(\Sigma)$ in $\Sigma \times \mathcal{W}_{M}$ is always trivial and we will denote it by

$$
\left(\begin{array}{c}
\mathbb{C}^{2 n} \\
\downarrow \pi \\
\rho(D)
\end{array}\right)
$$

For any $z=e^{i \theta}(0 \leq \theta \leq 2 \pi)$, let $\mathbb{R}^{2 n}\left(e^{i \theta}\right)$ be the totally real subspace $\rho^{*} T_{\rho\left(e^{i \theta}\right)}\left(\bar{\Lambda}_{\phi_{0}}\right)$ of $T_{\rho\left(e^{i \theta}\right)} \mathbb{C}^{2 n}=\mathbb{C}^{2 n}$. Consider all $H^{1,2}$-sections $s: \Sigma \rightarrow \mathbb{C}^{2 n}$ such that $s\left(e^{i \theta}\right) \in \mathbb{R}^{2 n}\left(e^{i \theta}\right)$. The linearized operator of $\rho$ is given by

$$
\bar{\partial}_{z}: H^{1,2}\left(\Sigma, \mathbb{C}^{2 n}\right) \rightarrow L^{2}\left(\Sigma, \mathbb{C}^{2 n}\right) .
$$

This is a Fredholm operator, so we can compute its index

$$
\operatorname{index}\left(\bar{\partial}_{z}\right)=\operatorname{dim} \operatorname{Ker}\left(\bar{\partial}_{z}\right)-\operatorname{dim} \operatorname{Coker}\left(\bar{\partial}_{z}\right) .
$$

This indice is invariant under deformation of holomorphic disks. If the normal Maslov indice of $\rho$ is denoted by $\mu\left(\bar{\partial}_{z}\right)$, then we have the following (cf. [32]):

$$
\operatorname{indice}\left(\bar{\partial}_{z}\right)=\mu\left(\bar{\partial}_{z}\right)+2 n=2 n \text {. }
$$


Thus the kernel of $\bar{\partial}_{z}$ is of dimension at least $2 n$. Recall that a holomorphic disk $\rho$ is regular in the sense of the Fredholm theory if the cokernel of $\bar{\partial}_{z}$ vanishes, equivalently, the kernel has dimension exactly equal to $2 n$.

For every disk $\rho:(\Sigma, \partial \Sigma) \rightarrow\left(\Sigma \times \mathcal{W}_{M}, \bar{\Lambda}_{\phi_{0}}\right)$, we have a loop of $2 n$-dimensional real subspaces $\left\{\mathbb{R}^{2 n}\left(e^{i \theta}\right) \mid 0 \leq \theta \leq 2 \pi\right\}$ in $\mathbb{C}^{2 n}$. By fixing a real $\mathbb{R}^{2 n}$ subspace in $T_{\rho(1)} \mathcal{W}_{M}$, this induces a map from $\partial \Sigma$ to $G L(2 n, \mathbb{C}) / G L(2 n, \mathbb{R})$. In general, this map may not lift to a map from $\partial \Sigma$ to $G l(2 n, \mathbb{C})$. However, this property of being able to be lifted to $C^{\infty}(\partial \Sigma, G L(2 n, \mathbb{C}))$ is invariant under continuous deformation of the disk or the boundary conditions. A disk is called trivial if all real subspaces $\mathbb{R}^{2 n}\left(e^{i \theta}\right)$ are equal to a constant $2 n$-dimensional real subspace (independent of $e^{i \theta}$ ). For a trivial disk, its induced loop always admits a lifting to $G L(2 n, \mathbb{C})$. Therefore, if a disk is path-connected to a trivial disk, then its induced loop must admit a lifting to an map $C^{\infty}(\partial \Sigma, G L(2 n, \mathbb{C}))$. We call this an associated loop of the disk $\rho$. It is clear that associated loop is defined up to multiplication by $\mathcal{L}^{+} G L(2 n, \mathbb{C})$ on the left. Here $\mathcal{L} G L(2 n, \mathbb{C})=C^{\infty}(\partial \Sigma, G L(2 n, \mathbb{C}))$, and $\mathcal{L}^{+} G L(2 n, \mathbb{C}) \subset C^{\infty}(\partial \Sigma, G L(2 n, \mathbb{C}))$ is the set of loops which can be extended to a holomorphic map $C^{\infty}(\Sigma, G L(2 n, \mathbb{C}))$. In this paper, we only consider discs which are path connected to a trivial disc. For these holomorphic discs, it is natural to consider the partial indices which are independent of the lifting. According to [32, 16] and [21], using a special form of Birkhoff factorization, we have

Theorem A ${ }^{13}$ : Let $\tilde{\rho}: S^{1}=\partial \Sigma \rightarrow \mathbb{R}^{2 n}(\theta)$ be a loop of totally real $2 n$ dimensional sub-spaces in $\mathbb{C}^{2 n}$, which is induced by some holomorphic disk $\rho:(\Sigma, \partial \Sigma) \rightarrow\left(\Sigma \times \mathcal{W}_{M}, \bar{\Lambda}_{\phi_{0}}\right)$. Then one can represent this loop map as

$$
\tilde{\rho}(z)=\Theta(z) \Lambda(z)^{\frac{1}{2}} \cdot \mathbb{R}^{2 n}, \quad z \in \partial \Sigma=S^{1},
$$

where $\Theta(z) \in \mathcal{L} G L(2 n, \mathbb{C})$ and $\Lambda(z)$ is a diagonal matrix:

$$
\Lambda(z)=\left[z^{k_{1}}, z^{k_{2}}, \cdots, z^{k_{2 n}}\right], \forall z \in \partial \Sigma .
$$

Here $\left(k_{1}, k_{2}, \cdots, k_{2 n}\right)$ is called partial indices of the loop $\rho$. Moreover, these partial indices have the following properties:

1. Each individual partial indice is not invariant under continuous deformation. However, the total sum of all partial indices is precisely the normal Maslov invariant, so it is invariant under any continuous defomation. Thus,

$$
\sum_{i=1}^{2 n} k_{i}=\mu=0 .
$$

\footnotetext{
${ }^{13}$ For the generic Maslov indice, this theorem was first obtained by 14 in complex surface. It was generalized to all dimensions in [16] with the assumption that all of the partial indices are non-negative. This last restriction was removed in 21]. The present statement follows the format in [21].
} 
2. A disk is Fredholm regular if and only if all of its partial indices $\geq-1$.

Using this theorem, Oh was able to reduce the equation for kernel vectors into a scalar equation:

$$
u=\left\{\begin{array}{lll}
\frac{\partial \xi}{\partial z}=0, & \forall z \in \Sigma, \\
\xi(z)=z^{\frac{k_{i}}{2}} \cdot \mathbb{R}, & & \forall z \in S^{1} .
\end{array}\right.
$$

This equation has no solution when $k_{i} \leq-1$. For $k_{i} \geq 0$, this equation has exactly $k_{i}+1$ linearly independent solution while each solution is a polynomial in $z$ with degree $k_{i}$.

Theorem B Suppose $f$ is a regular disk whose partial indice decomposition $\left(k_{1}, k_{2}, \cdots, k_{2 n}\right)$ contains exactly $l(\in[0, n])$ number of partial indices which equals -1 . Then the kernel matrix of this disk has co-rank at least l everywhere in the interior of this disk.

This can be easily derived from [16] and [21].

\subsection{The universal moduli space is regular}

Define

$$
\mathcal{G}=\bigcup_{\phi_{0} \in C^{\infty}(\partial \Sigma, \mathcal{H})} \bar{\Lambda}_{\phi_{0}}
$$

and

$$
\Upsilon=\bigcup_{\phi_{0} \in C^{\infty}(\partial \Sigma, \mathcal{H})} \mathcal{M}_{\phi_{0}}
$$

where $\mathcal{M}_{\phi_{0}}$ is the moduli space of all holomorphic disks with vanishing normal Maslov indice:

$$
\rho:(\Sigma, \partial \Sigma) \rightarrow\left(\Sigma \times \mathcal{W}_{M}, \bar{\Lambda}_{\phi_{0}}\right) .
$$

Clearly, $\mathcal{G}$ is an infinite dimensional manifold and there is a natural projection $p: \Upsilon \rightarrow \mathcal{G}$ such that for any $\phi_{0} \in C^{\infty}(\partial \Sigma, \mathcal{H})$, the moduli space $\mathcal{M}_{\phi_{0}}$ is mapped to $\bar{\Lambda}_{\phi_{0}}$.

Recall that the Moduli space is smooth if every holomorphic disk in this Moduli space is regular. It follows from the following lemma and the Sard-Smale transversality theorem that $\mathcal{M}_{\phi_{0}}$ is smooth for a generic boundary value $\phi_{0}$.

Lemma 3.2.1. ${ }^{14}$ The universal moduli space $p: \Upsilon \rightarrow \mathcal{G}$ is smooth.

Proof. The tangent space of $\mathcal{G}$ at $\phi_{0}$ can be considered as

$$
T_{\phi_{0}} \mathcal{G}=C^{\infty}(\partial \Sigma \times M), \quad \forall \phi_{0} \in C^{\infty}(\partial \Sigma, \mathcal{H}) .
$$

\footnotetext{
${ }^{14}$ This was first carried out in $[22]$ in the context of Lagrange/totally real submanifold. For convenience of readers, we include a proof of this transversality below.
} 
Let $\epsilon_{k} \rightarrow 0$ be a sequence of positive numbers which converges to zero. Denote $\bar{\epsilon}=\left(\epsilon_{1}, \epsilon_{2}, \cdots\right)$. Set

$$
\|f\|_{\bar{\epsilon}}=\sum_{k=0}^{\infty} \epsilon_{k} \max _{x \in \partial \Sigma \times M}\left|D^{k} f(x)\right| .
$$

This defines an $\bar{\epsilon}$-norm on

$$
C^{\bar{\epsilon}}\left(\Lambda_{\phi_{0}}\right)=\left\{f \in C^{\infty}(\partial \Sigma \times M) \mid\|f\|_{\bar{\epsilon}}<\infty\right\} .
$$

This norm has been introduced by Floer in a different context and this is a Banach space. We can choose $\bar{\epsilon}$ so that $C^{\bar{\epsilon}}\left(\Lambda_{\phi_{0}}\right)$ is dense in $C^{\infty}(\partial \Sigma \times M)$ with respect to the $L^{2}$ norm.

Now fix $s>1$ and define

$$
\mathcal{F}=\mathcal{F}^{s}=H^{s+1}\left(\Sigma, \Sigma \times \mathcal{W}_{M}\right)
$$

which is a Sobolev space of all maps $\omega: \Sigma \rightarrow \Sigma \times \mathcal{W}_{M}$ whose $(s+1)^{t h}$ derivatives are in $L^{2}$. For any boundary map $\phi_{0}: \partial \Sigma \rightarrow \mathcal{H}$, the totally real submanifold $\Lambda_{\phi_{0}}$ of $\Sigma \times \mathcal{W}_{M}$ is a point in $\mathcal{G}$. For any small $r$ positive, we define an $r$ neighborhood of this point in $\mathcal{G}$ as:

$$
\mathcal{N}\left(\Lambda_{\phi_{0}}\right)=\left\{\Lambda_{\phi_{0}+f} \mid\|f\|_{\bar{\epsilon}}<r \text { and } f \in C^{\infty}(\partial \Sigma \times M)\right\} .
$$

The corresponding neighborhood of holomorphic disks is

$$
\begin{aligned}
\overline{\mathcal{M}} & =\mathcal{M}\left(\mathcal{N}\left(\Lambda_{\phi_{0}}\right)\right) \\
& =\left\{\left(\rho, \Lambda_{\phi_{0}+f}\right)|\bar{\partial} \rho=0, \rho|_{\partial \Sigma} \subset \Lambda_{\phi_{0}+f},\|f\|_{\bar{\epsilon}}<r\right\} .
\end{aligned}
$$

For each $\rho \in \mathcal{F}$, define the pulled back bundle as

$$
\mathcal{B}_{\rho}=H^{s}\left(\rho^{*} T \mathcal{W}_{M}\right)
$$

consisting of all $H^{s}$-sections of $\rho^{*} T \mathcal{W}_{M}$ on $\Sigma$. Set

$$
\mathcal{B}=\bigcup_{\rho \in \mathcal{F}} \mathcal{B}_{\rho}=\bigcup_{\rho \in \mathcal{F}} H^{s}\left(\rho^{*} T \mathcal{W}_{M}\right)
$$

This is a smooth bundle over $\mathcal{F}$. We further set

$$
\Omega\left(\Lambda_{\phi_{0}}\right):=H^{s+\frac{1}{2}}\left(\partial \Sigma, \Sigma \times \mathcal{W}_{M}\right) \cap C^{0}\left(\partial \Sigma, \Lambda_{\phi_{0}}\right) .
$$

This is simply the space of $H^{s+\frac{1}{2}}$ maps from $\partial \Sigma$ to $\Lambda_{\phi_{0}}$. By the trace theorem, for each map $\rho \in H^{s+1}\left(\Sigma, \Sigma \times \mathcal{W}_{M}\right)$, its boundary map $\left.\rho\right|_{\partial \Sigma}$ lies in $\Omega\left(\Sigma \times \mathcal{W}_{M}\right)=H^{s+\frac{1}{2}}\left(\partial \Sigma, \Sigma \times \mathcal{W}_{M}\right)$. Now we define a map

$$
\triangle: \mathcal{F} \times \mathcal{N}\left(\Lambda_{\phi_{0}}\right) \rightarrow \mathcal{B} \times \Omega\left(\Lambda_{\phi_{0}}\right)
$$

by

$$
\triangle\left(\rho, \Lambda_{\phi_{0}+f}\right)=\left(\bar{\partial} \rho, \phi_{\phi_{0}+f}^{-1}\left(\left.\rho\right|_{\partial \Sigma}\right)\right)
$$


where $\phi_{\phi_{0}+f}: \Lambda_{\phi_{0}} \rightarrow \Lambda_{\phi_{0}+f}$ identifies the small perturbation $\Lambda_{\phi_{0}+f}$ with $\Lambda_{\phi_{0}}$. Denote by

$$
X_{f}=\left.\frac{d}{d t}\left(\phi_{\phi_{0}+t f}^{-1}\right)\right|_{t=0} \in T_{\Lambda_{\phi_{0}}} \mathcal{N}\left(\Lambda_{\phi_{0}}\right) .
$$

Consequently, $T_{\Lambda_{\phi_{0}}} \mathcal{N}\left(\Lambda_{\phi_{0}}\right)$ consists of all such fields $X_{f}$ for $f \in C^{\infty}(\partial \Sigma \times M)$. Note that

$$
\overline{\mathcal{M}}=\triangle^{-1}\left(\{0\} \times \Omega\left(\Lambda_{\phi_{0}}\right)\right) .
$$

The goal here is to show that the map $\triangle$ is transverse to the submanifold at $\left(\{0\} \times \Omega\left(\Lambda_{\phi_{0}}\right)\right) \subset$ $\mathcal{B} \times \Omega\left(\Sigma \times \mathcal{W}_{M}\right)$. Then it follows that $\overline{\mathcal{M}}$ is a smooth Banach submanifold of $\mathcal{F}^{s} \times \mathcal{N}\left(\Lambda_{\phi_{0}}\right)$. Moreover, by the elliptic regularity theory, $\overline{\mathcal{M}}$ is a smooth Banach submanifold of $\mathcal{F}^{s} \times \mathcal{N}\left(\Lambda_{\phi_{0}}\right)$ for all $s>1$.

For any small $f \in C^{\infty}(\partial \Sigma \times M)$, we set $\phi=\phi_{\phi_{0}+f}$ for simplicity. To show the transversality, we need to show

$$
I_{\triangle} T_{\rho, \Lambda_{\phi_{0}+f}}\left(\mathcal{F} \times \mathcal{N}\left(\Lambda_{\phi_{0}}\right)\right)+\{0\} \oplus T_{\phi^{-1}\left(\left.\rho\right|_{\partial \Sigma}\right)} \Omega\left(\Lambda_{\phi_{0}}\right)=T_{0, \phi^{-1}\left(\left.\rho\right|_{\partial \Sigma}\right)}\left(\mathcal{B} \times \Omega\left(\partial \Sigma \times \mathcal{W}_{M}\right)\right),
$$

where $\left(\rho, \Lambda_{\phi_{0}+f}\right) \in \mathcal{F} \times \mathcal{N}\left(\Lambda_{\phi_{0}}\right)$. If $\left(\xi, X_{f}\right) \in T_{\rho, \Lambda_{\phi_{0}+f}}\left(\mathcal{F} \times \mathcal{N}\left(\Lambda_{\phi_{0}}\right)\right)$, then a straightforward calculation shows that

$$
\operatorname{Im}_{\triangle}\left(\xi, X_{f}\right)=\left(\bar{\partial} \xi, X_{f}-\left.\xi\right|_{\partial \Sigma}\right) .
$$

Clearly, the LHS (left hand side) of (3.1) is a subspace of the RHS (right hand side). We need to show that the normal space to LHS in the RHS of (3.1) is null. Suppose that $(r, \alpha)$ is in such an normal space, that is, $(r, \alpha) \perp m_{\triangle}\left(\xi, X_{f}\right)$ and $(r, \alpha) \perp\left(\{0\} \bigoplus T_{\phi^{-1}\left(\left.\rho\right|_{\partial \Sigma}\right)} \Omega\left(\Lambda_{\phi_{0}}\right)\right)$. The second condition implies

$$
\alpha \in\left(T_{\phi^{-1}\left(\left.\rho\right|_{\partial \Sigma}\right)} \Omega\left(\Lambda_{\phi_{0}}\right)\right)^{\perp} .
$$

In other words, $\alpha$ represents some variation normal to $T_{\phi_{\phi_{0}}^{-1}\left(\left.\rho\right|_{\partial \Sigma}\right)} \Omega\left(\Lambda_{\phi_{0}}\right)$. The first condition implies that

$$
\int_{\Sigma}(\bar{\partial} \xi, r)+\int_{\partial \Sigma}\left(X_{f}-\left.\xi\right|_{\partial \Sigma}, \alpha\right)=0
$$

Integrating by parts, we have

$$
\int_{\Sigma}\left(\xi, \nabla_{J} r\right)+\int_{\partial \Sigma}\left(\left.\xi\right|_{\partial \Sigma}, e^{-i \theta} r-\alpha\right) d \theta+\int_{\partial \Sigma}\left(X_{f}-\left.\xi\right|_{\partial \Sigma}, \alpha\right) d \theta=0 .
$$

Thus

$$
\begin{aligned}
\nabla_{J} r & =0 \\
-\left.\alpha\right|_{\partial \Sigma}+\left.e^{-i \theta} r\right|_{\partial \Sigma} & =0 \\
\alpha^{\perp} & =0 .
\end{aligned}
$$

Equation (3.4) shows that $\alpha$ must be tangent to $T_{\phi^{-1}\left(\left.\rho\right|_{\partial \Sigma}\right)} \Omega\left(\Lambda_{\phi_{0}}\right)$, but $\alpha$ must be also normal to this space, so $\alpha=0$. Consequently, $\left.r\right|_{\partial \Sigma}=0$ by (3.3). This, together with (3.2), implies that $r=0$ in $\Sigma$. This completes the proof of transversality. 
The same arguments also show that for a generic path $\psi:[0,1] \times \partial \Sigma \mapsto \mathcal{H}$, the total moduli $\bigcup_{t \in[0,1]} \mathcal{M}_{\mid} \psi(t, \cdot)$ is smooth.

\subsection{Selection of a generic path}

Next we turn our attention to variations of an arbitrary disk $f$ in the universal moduli space of holomorphic disks. As before, for every disk, it induces a map from $\partial \Sigma$ to the space of totally real $2 n$ plane in $\mathbb{C}^{2 n}$. Since all disks concerned are path connected to a trivial disk, this induced map can be lifted to a map from the universal moduli space of holomorphic disks to the loop space $\mathcal{L} G L(2 n, \mathbb{C})$. It is well defined up to some normalization of the induced normal bundle of $\rho^{*} T_{w} \mathcal{W}_{M}$ over $\Sigma$. In other words, it is a map from a holomorphic disk to $\mathcal{L} G L(2 n, \mathbb{C}) / \mathcal{L}^{+} G L(2 n, \mathbb{C})$. Define a fiber bundle $\mathcal{C}$ over $\mathcal{F}$ such that each fibre is isomorphic to $\mathcal{L} G L(2 n, \mathbb{C}) / \mathcal{L}^{+} G L(2 n, \mathbb{C})$. This defines a natural map from the universal moduli space $\overline{\mathcal{M}}$ to this fibre bundle

$$
\star: \mathcal{G} \rightarrow \mathcal{C}
$$

which simply maps each holomorphic disk to its associated loop in $\mathcal{L} G l(2 n, \mathbb{C}) / \mathcal{L}^{+} G l(2 n, \mathbb{C})$.

It is well known that $\mathcal{L} G L(2 n, \mathbb{C}) / \mathcal{L}^{+} G L(2 n, \mathbb{C})$ admits a smooth stratification of loops by its partial indice $k=\left(k_{1}, k_{2}, \cdots, k_{2 n}\right)$. A somewhat lengthy calculation ${ }^{15}$ shows

Lemma 3.3.1. For the smooth stratification of $\mathcal{L} G L(2 n, \mathbb{C}) / \mathcal{L}^{+} G L(2 n, \mathbb{C})$ by its partial indices $k=\left(k_{1}, k_{2}, \cdots k_{2 n}\right)$, the real codimension of each component indexed by $k$ is

$$
d=\sum_{i=1}^{2 n} \sum_{j=i+1}^{2 n}\left(k_{i}-k_{j}-\lambda_{i j}\right)
$$

where $k_{1} \geq k_{2} \geq \cdots \geq k_{2 n}$ and

$$
\lambda_{i j}= \begin{cases}1 & \text { if } k_{i}>k_{j} \text { and } i<j \\ 0 & \text { Otherwise. }\end{cases}
$$

Let $S_{0}, S_{1}, S_{2}, S$ be the set of loops whose partial indices satisfy:

1. All partial indices in $S_{0}$ are equal to 0 ;

2. all partial indices are of the form $(1,0, \cdots, 0,-1)$ in $S_{1}$;

3. at least two of the partial indices equal to -1 in $S_{2}$, but no partial indice is $\leq-2$;

4. at least one partial indice in $S$ is less or equal to -2 .

\footnotetext{
${ }^{15}$ Proof of this Lemma can be founded in Appendix.
} 
According to Lemma 3.3.1. $S_{0}$ is in generic position, while the real codimension for $S_{1}$ is 1 . For $S \subset \mathcal{L} G L(2 n, \mathbb{C}) / \mathcal{L}^{+} G L(2 n, \mathbb{C})$, suppose that $k_{j} \geq k_{2 n}$ and $k_{2 n} \leq-2, \forall i \in[1,2 n]$. Then the codimension is:

$$
\begin{aligned}
d & =\sum_{i=1}^{2 n} \sum_{j=i+1}^{2 n}\left(k_{i}-k_{j}-\lambda_{i j}\right) \\
& \geq \sum_{i=1}^{2 n}\left(k_{i}-k_{2 n}-\lambda_{i(2 n)}\right) \\
& =\sum_{i=1}^{2 n} k_{i}+\sum_{i=1}^{2 n}\left(-k_{2 n}\right)-\sum_{i=1}^{2 n} \lambda_{i(2 n)} \\
& \geq 0+\sum_{i=1}^{2 n} 2-\sum_{i=1}^{2 n-1} 1=2 n+1 .
\end{aligned}
$$

For $S_{2}$, we can assume $k_{2 n-1}=k_{2 n}=-1$. Thus,

$$
\begin{aligned}
d & =\sum_{i=1}^{2 n} \sum_{j=i+1}^{2 n}\left(k_{i}-k_{j}-\lambda_{i j}\right) \\
& \geq \sum_{i=1}^{2 n-1}\left(k_{i}-k_{2 n-1}-\lambda_{i(2 n-1)}\right)+\sum_{i=1}^{2 n-2}\left(k_{i}-k_{2 n}-\lambda_{i(2 n)}\right) \\
& =2 \sum_{i=1}^{2 n-2} k_{i}+\sum_{i=1}^{2 n-2}\left(-k_{2 n}-k_{2 n-1}\right)-\sum_{i=1}^{2 n-2}\left(\lambda_{i(2 n)}+\lambda_{i(2 n-1)}\right) \\
& =2\left(k_{2 n-1}+k_{2 n}\right)+\sum_{i=1}^{2 n-2}\left(-k_{2 n}-k_{2 n-1}\right)-\sum_{i=1}^{2 n-2}\left(\lambda_{i(2 n)}+\lambda_{i(2 n-1)}\right) \\
& \geq 4+2(2 n-2)-2(2 n-2)=4 .
\end{aligned}
$$

According to Lemma 3.3 .1 we have

$$
\mathcal{L} G L(2 n, \mathbb{C}) / \mathcal{L}^{+} G L(2 n, \mathbb{C})=S_{0} \bigcup S_{1} \bigcup S_{2} \bigcup S=S_{0} \bigcup\left(S_{1}^{\text {a.s. }} \bigcup S_{1}^{\text {n.a.s. }}\right) \bigcup S_{2} \bigcup S .
$$

Here $S_{1}^{a . s .}$ denotes all of the holomorphic disks in $S_{1}$ which are super regular at $z=0$ and

$$
S_{1}=S_{1}^{a . s .} \bigcap S_{1}^{\text {n.a.s }}
$$

It is straightforward to check that $S_{1}^{\text {n.a.s. }}$ has at least codimension 1 in $S_{1}$.

Proposition 3.3.2. This map $\star$ is a submersion at any embedded disk of $\overline{\mathcal{M}}$. 
Proof. We need to show that $\star$ is a submersion at a regular disk or at a non-regular but embedded disk. The first assertion follows from the fact that any regular holomorphic disk $f$ with boundary in a totally real submanifold is stable under a small deformation of the boundary map. To be more explicit, we let $f:(\Sigma, \partial \Sigma) \rightarrow\left(\Sigma \times \mathcal{W}_{M}, \Lambda_{\phi_{0}}\right)$ be a holomorphic disk with vanishing normal Maslov indice. If $f$ is regular in the sense of Fredholm theory, then there is a holomorphic disk $f+\delta f$ such that its boundary lies in some $\mathbb{R}^{2 n}\left(e^{i \theta}\right)+\delta P\left(e^{i \theta}\right)$. For this family of holomorphic disks, the associated loop is exactly $\ell+\delta \ell$. Thus, " $\star$ " is an submersion at the image (under $\star$ ) of every regular holomorphic disk.

Now suppose that $\star(f)=\ell \in S \subset \mathcal{C}$. Since $\mathcal{C}$ is a smooth infinite dimensional manifold which admits a smooth stratification by partial indices. More specifically, the space of loop matrices in $G l(2 n, \mathbb{R})$, it can be decomposed as the union of $S_{0} \bigcup S_{1} \bigcup S_{2} \bigcup S$. Here we are only considering $S$ which lie in a connected component of $S_{1} \bigcup S_{1} \bigcup S_{2}$. Therefore, there always exists a path $\delta \ell(t)$ such that $\delta \ell(0)=0$; and $\ell+\delta \ell(t) \in \mathcal{C} \backslash S$ when $t \neq 0$. Consider

$$
f:(\Sigma, \partial \Sigma) \rightarrow\left(\Sigma \times \mathcal{W}, \bigcup_{\theta \in S^{1}}(\theta, L(\theta))\right)
$$

Here $L(\theta)$ is a totally real sub-manifold in $(\theta, \mathcal{W})$ for any $\theta \in S^{1}$. At the tangential level, $T_{f(\theta)} \mathcal{W}_{M}$ is a trivial $\mathbb{C}^{2 n}$ bundle over $\Sigma$. Using this trivialization, we may assume

$$
T_{f(\theta)} L(\theta)=\mathbb{R}^{2 n}(\theta)=A(\theta) \cdot \mathbb{R}^{2 n}
$$

for some $\mathbb{R}^{2 n}$ fixed in $T_{f(1)} \mathcal{W}_{M}$. Here $A(\theta) \in G l(2 n, \mathbb{C})$. Clearly, $\ell$ can be lifted up to be a loop in $G l(2 n, \mathbb{C})$ and

$$
\ell(\theta)=A(\theta), \forall \theta \in S^{1} .
$$

The tangent space of $L G L(2 n, \mathbb{C})$ at $\ell$ can be represented by a smooth 1-parameter family of loops of matrices $(-\epsilon \leq t \leq \epsilon)$ :

$$
\ell(t, \theta)=A(\theta)(I+t B(\theta)), \quad \forall \theta \in S^{1} .
$$

The surjectivity at $f$ is equivalent to there being a pre-image of this path $\ell(t, \theta)$ for an arbitrary loop matrix $B$. Near a small tubular neighborhood of $L(\theta) \in \mathcal{W}_{\theta}$, we define a product metric (so $L(\theta)$ becomes totally geodesic in $\mathcal{W}_{M}$ ). Call this metric $g_{\theta}$. Define

$$
L(t, \theta)=\exp _{f(\theta), g_{\theta}}\left((A(\theta)(I+t B(\theta))) \cdot \mathbb{R}^{2 n}\right),
$$

where $(A(\theta)(I+t B(\theta))) \cdot \mathbb{R}^{n}$ represents the $\mathrm{n}$-dimensional plane spanned by it in $T_{f(\theta)} \mathcal{W}_{M}$. Clearly, $L(0, \theta)=L(\theta)$. Define $f(t)$ to be a family of disks in the total moduli space

$$
f(t):(\Sigma, \partial \Sigma) \rightarrow\left(\Sigma \times \mathcal{W}, \bigcup_{\theta \in S^{1}}(\theta, L(t, \theta))\right.
$$


such that the image of each $f(t)$ is identified with $f$, but they represent a 1-parameter path of holomorphic disks in the total moduli space. Clearly,

$$
\star(f(t))=\ell(t) .
$$

In other words, the map $\star$ is transversal to $S \subset \mathcal{L} G L(2 n, \mathbb{C}) / \mathcal{L}^{+} G L(2 n, \mathbb{C})$.

Next we want to use this submersion map $\star$ to calculate the codimension of various components of the universal moduli space.

Note that $\star^{-1} S_{0}, \star^{-1} S_{1}, \star^{-1} S_{2}$ and $\star^{-1} S$ are smooth manifold or submanifold in $\overline{\mathcal{M}}$, where $\star^{-1} S_{0}$ are the set of all super regular disks which is generic in $\overline{\mathcal{M}}, \star^{-1} S_{1}, \star^{-1} S_{2}$ are submanifolds of regular holomorphic disks in $\overline{\mathcal{M}}$ with real codimension at least 1 and 4 . Finally, $\star^{-1} S$ is the smooth submanifold of all irregular disks in $\overline{\mathcal{M}}$ with real codimension at least $2 n+1$. This, together with the remark at the end of last section, implies

Theorem 3.3.3. For any path $\psi:[0,1] \rightarrow C^{\infty}(\partial \Sigma, \mathcal{H})$ such that $\mathcal{M}_{\psi(0, \cdot)}$ contains a super regular disk with vanishing normal Maslov invariant, there exists a generic path (we still denote it by $\psi)$, which is arbitrarily close to the original path, such that the total moduli $\bigcup_{0 \leq s \leq 1}\{s\} \times \mathcal{M}_{\psi(s, \cdot)}$ is a smooth $2 n+1$-dimensional manifold. Moreover, there is a connected component $\mathcal{M}_{\psi}^{0}$ of this total moduli containing the super-regular disk in initial moduli $\mathcal{M}_{\psi(0, \cdot)}$, such that the followings hold:

1. The set of disks with partial indices $(0,0, \cdots, 0)$ in $\mathcal{M}_{\psi}^{0}$ is open and dense in this connected component;

2. The set of disks with partial indices $(1,0,0, \cdots, 0,-1)$ has codimension at least 1 in $\mathcal{M}_{\psi}^{0}$. The set of disks with partial indice $(1,0, \cdots, 0,-1)$ but not super-regular at $z=0$ has codimension 2 and higher;

3. The set of all other holomorphic disks has codimension 2 and higher;

4. There exist at most finitely many non-regular disks in the total moduli.

\subsection{Almost super regular foliations}

In this subsection, we first introduce the notion of an almost super regular foliation. This is a stronger notion compared to the nearly smooth foliation introduced earlier. Recall the natural projection $\pi: \mathcal{W}_{[\omega]} \rightarrow M$. The tangent space of $\mathcal{W}_{[\omega]}$ naturally splits into $T M$ union with tangent space to the fibre space of $T^{*} M$. A regular disk $f$ in $\mathcal{M}_{\phi_{0}}$ is called super regular at $z \in \Sigma \times M$ if the Jacobi map of $\pi \circ \mathrm{ev}$ is non-singular at $z \in \Sigma$, where ev $: \Sigma \times \mathcal{W}_{[\omega]} \mapsto \mathcal{W}_{[\omega]}$ maps $(z, f)$ to $f(z)$. It is called super regular if it is super regular at every point of $\Sigma$. It is called almost super regular if the Jacobi map of $\pi \circ \mathrm{ev}$ is nowhere vanishing in $\Sigma^{0}$. 
Definition 3.4.1. For any boundary map $\phi_{0} \in C^{\infty}(\partial \Sigma, \mathcal{H})$, an open and connected $2 n$ dimensional subset $\mathcal{U}_{\phi_{0}} \subset \mathcal{M}_{\phi_{0}}$ is called an almost super regular foliation if

1. it is a nearly smooth foliation (cf. Definition 1.4.2);

2. Every disk in $\overline{\mathcal{U}}_{\phi_{0}}$ is regular except perhaps at most a set of finitely many disks. Furthermore, the set of almost super regular disks has co-dimension at least 1, while the set of all disks of other types has at least co-dimension 2 or higher.

Clearly, for any boundary map $\phi_{0} \in C^{\infty}(\partial \Sigma, \mathcal{H})$, an almost super regular foliation $\mathcal{F}_{\phi_{0}}$ is super regular if $\mathcal{U}_{\phi_{0}}=\overline{\mathcal{U}}_{\phi_{0}}=\mathcal{M}_{\phi_{0}}$.

Proposition 3.4.2. If $\mathcal{F}_{\phi_{0}}$ is an almost super regular foliation, then $\Sigma \times \overline{\mathcal{U}}_{\phi_{0}}$ induces a foliation in $\Sigma^{0} \times M$ via the composition map of $\pi$ and ev, except at most a set of codimension 2 .

Proof. Self evident.

Corollary 3.4.3. For an almost super regular foliation, two disks intersect at most at subset of of $\Sigma \times M$ with codimension 2 or higher.

This in turns implies

Corollary 3.4.4. For any almost smooth solution $\phi$ of (1.1) which corresponds to an almost super regular foliation, the leaf vector field $X$ which annulate the Levi form $\pi_{2}^{*} \omega_{0}+\sqrt{-1} \partial \bar{\partial} \phi$ is smooth in $\tilde{\mathcal{V}}_{\phi_{0}}$ and is uniformly bounded in $\Sigma^{0} \times M$.

Proposition 3.4.5. For a generic boundary map $\phi_{0}: \partial \Sigma \rightarrow \mathcal{H}$ such that every embedded disk in $\mathcal{M}_{\phi_{0}}$ is regular, then a nearly smooth foliation is necessary almost super regular. Moreover, the connected component $\overline{\mathcal{U}}_{\phi_{0}}$ is a smooth manifold without boundary.

Proof. For any sequence of holomorphic disks $f_{k} \in \mathcal{U}_{\phi_{0}}$, the leaf vector field $X_{k}$ has uniform upper bound. It follows that there is a subsequence (which we still denoted as $\left\{f_{k}, k \in \mathcal{I}\right\}$ ), such that converges to a limiting embedded disk $f_{\infty} \in \mathcal{M}_{\phi_{0}}$. By our assumption, this limiting disk must be regular in the sense of Fredholm theory. In particular, $f_{\infty}$ is an interior point of $\mathcal{M}_{\phi_{0}}$. Consequently, $\overline{\mathcal{U}}_{\phi_{0}}$ is compact without boundary.

Conversely,

Proposition 3.4.6. For a boundary map $\phi_{0}: \partial \Sigma \rightarrow \mathcal{H}$ such that all but possibly finitely many disks in $\mathcal{M}_{\phi_{0}}$ are regular. Define $\tilde{\mathcal{U}}_{\phi_{0}}$ to be the set of all super regular and all almost super regular disks. Suppose

1. $\overline{\mathcal{U}}_{\phi_{0}} \backslash \tilde{\mathcal{U}}_{\phi_{0}}$ has codimension 2 or higher;

2. The evaluation map is continuous on $\overline{\mathcal{U}}_{\phi_{0}}$. 
Then $\mathcal{U}_{\phi_{0}}$ defines an almost super regular foliation. In particular, the covering indice for evaluation map is 1 .

Proof. Consider the evaluation of $N$ in the central fibre $\{0\} \times M$. The evaluation map is locally covering map from generic points in $N$. Since $\partial N$ is a set of isolated singular disks and the evaluation map is continuous at this set, then the image of $N$ must be $\{0\} \times M$ entirely. Since $M$ is connected, the covering indice must be some positive constant $k \geq 1$ for generic point. In particular, $\mathcal{U}_{\phi_{0}}$ must be decomposed into $k$ disjoint connected component. And each connected component gives rise to a nearly smooth foliation. However, a nearly smooth foliation is unique. Thus, there is only one connected component and covering indice is 1 .

Now, we introduce the notion of partially smooth foliation, which arises from limits of almost super regular foliations under convergence of boundary maps in suitable norms.

Definition 3.4.7. For any boundary map $\phi_{0} \in C^{\infty}(\partial \Sigma, \mathcal{H})$, an open $2 n$ dimensional subset $\mathcal{U}_{\phi_{0}} \subset \mathcal{M}_{\phi_{0}}$ and closed subset $\tilde{\mathcal{U}}_{\phi_{0}} \subset \mathcal{M}_{\phi_{0}}$ is called a partially smooth foliation if the following conditions are met:

1. $\mathcal{U}_{\phi_{0}} \subset \overline{\mathcal{U}}_{\phi_{0}} \subset \tilde{\mathcal{U}}_{\phi_{0}}$.

2. Every disk in $\mathcal{U}_{\phi_{0}}$ is super regular.

3. The evaluation map $\pi \circ \mathrm{ev}: \Sigma \times \overline{\mathcal{U}}_{\phi_{0}} \rightarrow \Sigma \times M$ is a continuous onto map into its image where the image is dense in $\partial \Sigma \times M$ Moreover, the image of $\tilde{\mathcal{U}}_{\phi_{0}}$ is $\Sigma \times M$.

4. Any disk in $\mathcal{U}_{\phi_{0}}$ doesn't intersect with any other disk in $\tilde{\mathcal{U}}_{\phi_{0}}$ in $\Sigma^{0} \times M$.

Recall that an almost smooth solution of the HCMA equation (1.1) corresponds to a nearly smooth foliation. One can view a partially smooth foliation as a sequential limit of nearly smooth foliations, while a partially smooth solution can be viewed as a sequential limit of almost smooth solutions. In this regard, a partially smooth solution corresponds conceptually to a partially smooth foliation.

\subsubsection{Open-denseness of almost super regular disks}

In this subsection, we reformulate Theorem 3.3 .3 in terms of a.s.r or s.r. holomorphic disks. Let us first describe some properties of holomorphic disks with either partial indices $(0,0, \cdots, 0)$ or $(1,0, \cdots 0,-1)$.

Theorem 3.4.8. Given a connected component of $\mathcal{M}_{\phi_{0}}$ which consists of holomorphic disks with partial indices $(0,0, \cdots, 0)$. If there exists at least one super regular disk in this connected component, then all disks in this component are super regular. 
Proof. The set of super regular disks is open in the moduli space. Therefore, we just need to show that it is close among disks with partial indices $(0,0, \cdots, 0)$. Let $\left\{f_{i}:(\Sigma, \partial \Sigma) \rightarrow\left(\Sigma \times \mathcal{W}_{M}, \bar{\Lambda}_{\phi_{0}}\right)\right\}$ be a sequence of super regular disks such that $f_{i} \rightarrow f$ smoothly (cf. in $C^{2, \alpha}\left(\Sigma, \Sigma \times \mathcal{W}_{M}\right)$ - norm ${ }^{16}$ ) in $\mathcal{M}_{\phi_{0}}$. We want to prove that if $f$ has partial indices $(0,0, \cdots, 0)$, then $f$ is also super regular.

Since $f$ is regular, there is a small neighborhood $\mathcal{O}_{f} \subset \mathcal{M}_{\phi_{0}}$ of $f$ such that ev $: \Sigma \times \mathcal{O}_{f} \rightarrow$ $\Sigma \times \mathcal{W}_{M}$ is smooth. Put $F=\pi \circ e v$. Let $t_{1}, t_{2}, \cdots, t_{2 n}$ be local coordinates of $\mathcal{O}_{f}$, write

$$
s_{k}^{(i)}(z)=\left.\frac{\partial e v}{\partial t_{k}}\right|_{f_{i}(z)} \in T_{f_{i}(z)}^{1,0} \mathcal{W}_{M}, \quad 1 \leq k \leq 2 n .
$$

Then, $\left\{s_{k}\right\}_{k=1}^{2 n}$ is a basis of the kernel space of the $\bar{\partial}$ operator. Moreover, at each image point $f(z)$, the set of $2 n$ vertical vectors $\left\{s_{k}\right\}_{k=1}^{2 n}$ is also a basis of the "vertical" tangential subspace $T_{f(z)} \mathcal{M}_{\phi_{0}}$. Since $\mathcal{W}_{M}$ is locally the same as $T^{*} M$, its tangent space naturally splits into a $T M$ part and the tangent space of the fibre direction. Denote by $\left(\begin{array}{l}u \\ v\end{array}\right)$ the corresponding two components of any kernel vector, where $u, v \in C^{n}$. Set the k-th kernel vector as

$$
s_{k}^{(i)}=\left(\begin{array}{c}
u_{k}^{(i)} \\
v_{k}^{(i)}
\end{array}\right), \quad 1 \leq k \leq 2 n .
$$

Note that $u_{k}^{(i)}$ is clearly a vector in $T^{1,0} M$, while $v_{k}^{(i)}$ may depends on the choice of local Kähler potentials. According to Proposition 2.3.4 there exists a solution $\phi^{(i)}$ of equation (1.1) in $\pi \circ e v\left(\Sigma \times \mathcal{O}_{f}\right)$ with $\left.\phi^{(i)}\right|_{\partial \Sigma \times M}=\left.\phi_{0}\right|_{\partial \Sigma \times M}$. By Proposition 2.3.8, there is a uniform $C$ such that

$$
\left|\partial \bar{\partial} \phi^{i}\right| \leq C
$$

For any point $(z, x)$ in the image of $\pi \circ \operatorname{ev}(z, f)$, let $U$ be a small open set of $x$ in $M$. Then

$$
G^{(i)}=\left(g_{0, \alpha \bar{\beta}}+\frac{\partial^{2} \phi^{(i)}}{\partial w_{\alpha} \partial \bar{w}_{\beta}}\right)_{n \times n}>0, \quad S^{(i)}=\left(\frac{\partial^{2}\left(\rho+\phi^{(i)}\right)}{\partial w_{\alpha} \partial w_{\beta}}\right),
$$

where $\omega_{0}=g_{0, \alpha \bar{\beta}} d w^{\alpha} \wedge d \bar{w}^{\beta}=\sqrt{-1} \partial \bar{\partial} \rho$ in $U$. By a straightforward calculation, we have that for any $(z, w) \in f_{i}(\Sigma)$,

$$
\left(v_{k}^{(i)}\right)_{n \times 1}=G_{n \times n}^{(i)} \cdot\left(\bar{u}_{k}^{(i)}\right)_{n \times 1}+S_{n \times n}^{(i)} \cdot\left(u_{k}^{(i)}\right)_{n \times 1} .
$$

¿From this equation, it is clear that $v_{k}^{(i)}$ is not "tensorial" in the usual sense since $S$ is not. However,

$$
\operatorname{det}\left(\begin{array}{cccc}
u_{1}^{(i)} & u_{2}^{(i)} & \ldots & u_{2 n}^{(i)} \\
v_{1}^{(i)} & v_{2}^{(i)} & \cdots & v_{2 n}^{(i)}
\end{array}\right)=\operatorname{det}\left(\begin{array}{cccc}
u_{1}^{(i)} & u_{2}^{(i)} & \ldots & u_{2 n}^{(i)} \\
\bar{u}_{1}^{(i)} & \bar{u}_{2}^{(i)} & \ldots & \bar{u}_{2 n}^{(i)}
\end{array}\right) \cdot \operatorname{det} G^{(i)}
$$

\footnotetext{
${ }^{16}$ This regularity assumption is not optimal.
} 
is both real and holomorphic. Note that the right hand side is a function independent of the choice of local coordinate in $M$. Thus, the left hand side is well defined in $\Sigma$ and it must be a global constant along the disc. Suppose this constant $c_{i}{ }^{17}$ and

$$
\operatorname{det}\left(\begin{array}{cccc}
u_{1}^{(i)} & u_{2}^{(i)} & \cdots & u_{2 n}^{(i)} \\
\bar{u}_{1}^{(i)} & \bar{u}_{2}^{(i)} & \cdots & \bar{u}_{2 n}^{(i)}
\end{array}\right) \cdot \operatorname{det} G^{(i)}=c_{i} .
$$

A more global view of (3.7) is

$$
(\pi \circ e v)^{*} \omega_{\phi^{(i)}}^{n}=\left.\omega_{0}^{n}\right|_{z=z_{0} \in \partial \Sigma} .
$$

Since $f_{i}$ is a super regular disk, by definition, we have

$$
\operatorname{det}\left(\begin{array}{cccc}
u_{1}^{(i)} & u_{2}^{(i)} & \cdots & u_{2 n}^{(i)} \\
\bar{u}_{1}^{(i)} & \bar{u}_{2}^{(i)} & \cdots & \bar{u}_{2 n}^{(i)}
\end{array}\right) \neq 0
$$

holds everywhere in $f_{i}(\Sigma)$. Note that the left side of (3.8) is exactly the Jacobian of $\pi \circ \mathrm{ev}$. By our assumptions, $f$ is a disk with partial indices $(0,0, \cdots, 0)$, that is, that the kernel matrix is nowhere singular:

$$
\operatorname{det}\left(\begin{array}{cccc}
u_{1}^{(i)} & u_{2}^{(i)} & \cdots & u_{2 n}^{(i)} \\
v_{1}^{(i)} & v_{2}^{(i)} & \cdots & v_{2 n}^{(i)}
\end{array}\right) \neq 0
$$

everywhere in $f(\Sigma)$. What we need to prove is that the inequality (3.8) hold everywhere in $f(\Sigma)$. For $f_{i}(\Sigma)$, it is easy to see that This sequence of constants $\left\{c_{i}, i \in \mathcal{N}\right\}$ has both uniform upper and lower bounds, provided that the limiting disk $f$ has partial indices $(0,0, \cdots, 0)$. Since $\operatorname{det} G^{(i)} \leq C$, we deduce

$$
\operatorname{det}\left(\begin{array}{cccc}
u_{1} & u_{2} & \cdots & u_{2 n} \\
\bar{u}_{1} & \bar{u}_{2} & \cdots & \bar{u}_{2 n}
\end{array}\right)>0
$$

along the limiting disk $f$. This completes the proof of this theorem.

However, we can squeeze a little more out from the arguments above. Let $f$ be a holomorphic disk with partial indice $(1,0, \cdots, 0,-1)$ which is super regular at $z=0$. We claim that $f$ is almost super regular. In fact, the condition implies that

$$
\left.\operatorname{det}\left(\begin{array}{cccc}
u_{1}^{(i)} & u_{2}^{(i)} & \cdots & u_{2 n}^{(i)} \\
\bar{u}_{1}^{(i)} & \bar{u}_{2}^{(i)} & \cdots & \bar{u}_{2 n}^{(i)}
\end{array}\right)\right|_{z=0} \neq 0 .
$$

On the other hand, according to (3.6), for each $f_{i}$, we have

$$
c_{i}=\operatorname{det}\left(\begin{array}{cccc}
u_{1}^{(i)} & u_{2}^{(i)} & \cdots & u_{2 n}^{(i)} \\
v_{1}^{(i)} & v_{2}^{(i)} & \cdots & v_{2 n}^{(i)}
\end{array}\right)=\operatorname{det}\left(\begin{array}{cccc}
u_{1}^{(i)} & u_{2}^{(i)} & \cdots & u_{2 n}^{(i)} \\
\bar{u}_{1}^{(i)} & \bar{u}_{2}^{(i)} & \ldots & \bar{u}_{2 n}^{(i)}
\end{array}\right) \cdot \operatorname{det} G^{(i)}
$$

\footnotetext{
${ }^{17}$ When restricted to each $\left\{z_{0}\right\} \times M$, our normalization forces the first term on the right hand side to be positive.
} 
Using local deformation theory in next subsection (Corollary 4.2.11), $\log \operatorname{det} G^{(i)}$ is a subharmonic function in $\Sigma$. Moreover, it is uniformly bounded from above. Set

$$
h_{i}=\log \operatorname{det}\left(\begin{array}{cccc}
u_{1}^{(i)} & u_{2}^{(i)} & \cdots & u_{2 n}^{(i)} \\
\bar{u}_{1}^{(i)} & \bar{u}_{2}^{(i)} & \cdots & \bar{u}_{2 n}^{(i)}
\end{array}\right)
$$

along $f_{i}(\Sigma)$. Then, $\left\{h_{i}, i \in \mathbb{N}\right\}$ is a seequence of subharmonic functions on $f_{i}(\Sigma)$ which is uniformly bounded at $z=0$. Moreover, we have (cf. Proposition 4.2.4)

$$
\left|\Delta_{z} h_{i}\right|=\left|-\Delta_{z} \log \operatorname{det} G^{(i)}\right| \leq C, .
$$

holds in any compact subdomain of $\Sigma^{0}$. Then Harnack inequality for harmonic function implies that either $h_{i}$ approaches to $-\infty$ everywhere in any compact subset of $\Sigma^{0}$ or stays uniformly bounded in any compact subset of $\Sigma^{0}$. Since $h_{i}(0)$ is uniformly bounded, we have

$$
\lim _{i \rightarrow \infty} h_{i}(z)=h(z)=\left.\log \operatorname{det}\left(\begin{array}{cccc}
u_{1}^{(i)} & u_{2}^{(i)} & \cdots & u_{2 n}^{(i)} \\
\bar{u}_{1}^{(i)} & \bar{u}_{2}^{(i)} & \cdots & \bar{u}_{2 n}^{(i)}
\end{array}\right)\right|_{f(\Sigma)}>-\infty, \quad \forall z \in \Sigma^{0} .
$$

Consequently, $f$ is almost super regular. Thus we have proved

Theorem 3.4.9. If a holomorphic disk with partial indices $(1,0, \cdots, 0,-1)$ is super regular at one interior point and it can be connected to disks of partial indices $(0,0, \cdots, 0)$, then it is almost super regular.

In view of these two theorems, we can reinterpret Theorem 3.3 .3 as

Theorem 3.4.10. Given any path $\psi:[0,1] \rightarrow C^{\infty}(\partial \Sigma, \mathcal{H})$ such that $\mathcal{M}_{\psi(0, \cdot)}$ contains a super regular disk with vanishing Maslov disk, there exists a generic path (still denoted by $\psi$ for simplicity), which is arbitrarily close to the given path, such that for this new path, a connected component $\mathcal{M}_{\psi}^{0}$ of the total moduli space $\bigcup_{0 \leq s \leq 1}\{s\} \times \mathcal{M}_{\psi(s, \cdot)}$, which contains the initial super regular disk, is a smooth $2 n+1$-dimension manifold. Moreover, the followings hold

1. The set of super regular disks is open and dense in this connected component;

2. The set of almost super regular disks has codimension at least 1 in this component;

3. The set of disks, which are neither super regular nor almost super regular, has codimension at least 2;

4. There exist at most finitely many irregular disks in the total moduli space.

Moreover, there exist at most finitely many points $0<\bar{t}_{1}<\bar{t}_{2}<\cdots<\bar{t}_{l}<1$ such that for any $t \neq \bar{t}_{i}(1 \leq i \leq l)$, all disks in $\mathcal{M}_{\psi(t, \cdot)}$ are regular and its subset of disks in $\mathcal{M}_{\psi}^{0}$, which are neither super regular nor almost super regular, has codimension at least 2. When $t=\bar{t}_{i}$ for some $i, \mathcal{M}_{\psi(t, \cdot)} \cap \mathcal{M}_{\psi}^{0}$ may either contain some isolated irregular disks or a subset of disks which are neither super regular nor almost super regular which has exactly codimension 1. 


\subsubsection{Almost super regular foliations along a generic path}

In this subsection, we prove openness and closedness of almost super regular foliations along a generic path, which is assured by Theorem 3.4.10,

Theorem 3.4.11. Let $\psi:[0,1] \mapsto C^{\infty}(\Sigma, \mathcal{H})$ be a generic path with properties specified in Theorem 3.4.10. Suppose that $\mathcal{M}_{\psi(0, \cdot)} \cap \mathcal{M}_{\psi}^{0}$ is connected and gives rise to an almost super regular foliation, where $\mathcal{M}_{\psi}^{0}$ is the connected component defined in Theorem 3.4.10. Then for each $t, \mathcal{M}_{\psi(t, \cdot)} \cap \mathcal{M}_{\psi}^{0}$ is connected and induces a foliation in an open dense subset of $\Sigma \times M$. Moreover, this component gives rise to an almost super regular foliation except at most a finite number of times.

We firs prove

Lemma 3.4.12. For a sequence of $\tau_{i}, i \in \mathbb{N}\left(\lim _{i \rightarrow \infty} \tau_{i}=\bar{t} \in(0,1]\right)$ such that $\mathcal{F}_{\psi\left(\tau_{i}, \cdot\right)}$ is a sequence of almost smooth foliations. Suppose that $\phi_{i}$ is the corresponding sequence of almost smooth solutions and $\lim _{i \rightarrow \infty} \phi_{i}=\phi_{\infty}$. Then $\phi_{\infty}$ is a partially smooth solution of (1.1) and $\mathcal{R}_{\phi_{\infty}}$ is an open dense subset of $\Sigma \times M$. Moreover, there is a unique connected component of $\mathcal{M}_{\psi(\bar{t}, \cdot)}$ which is the limit of $\mathcal{F}_{\psi\left(\tau_{i}, \cdot\right)}$. Either this component is regular in which case there is an almost super regular foliation $\mathcal{F}_{\psi(t, \cdot)}$ for $t>\bar{t}$; or this component is an almost super regular foliation itself.

Proof. Following Theorem 5.0.17 after passing to a subsequence, $\mathcal{F}_{\psi\left(\tau_{i}, 0\right)}$ converges to a partially smooth foliation $\mathcal{F}_{\psi\left(\tau_{\infty}, \cdot\right)}$, where $\mathcal{U}_{\psi\left(\tau_{\infty}, \cdot\right)}$ denotes the set of all its super regular disks. Theorem 5.0 .15 implies that there is at least one super regular disk which is the limit of a sequence of super regular disks in $\mathcal{F}_{\phi_{0}\left(\tau_{i}\right)}$ with uniformly bounded capacity (cf. formula 5.2). Therefore, $\mathcal{U}_{\psi\left(\tau_{\infty}, \cdot\right)}$ is non-empty.

For convenience, let $\mathcal{B}$ be the union of all disks in $\mathcal{M}_{\psi\left(\tau_{\infty}, \cdot\right)}$ which are sequential limits of disks in $\mathcal{U}_{\phi_{0}\left(\tau_{i}\right)}$. By definition, any leaf in $\mathcal{B}$, is the limit of some sequence of disks in $\mathcal{M}_{\psi_{\tau_{i}}}$. Following Theorem 5.0.14 for any such sequence of disks, the corresponding sequence of leaf vector field in $T M$ has a uniform upper bound on length. In particular, all leaves in $\mathcal{B}$ have a uniform upper bound on the length of their leaf vector fields. Consequently, any sequence in $\mathcal{B}$ must have a convergent subsequence where the limit is an embedded disk in $\mathcal{M}_{\tau_{\infty}}$. It follows that $\mathcal{B}$ is a closed, bounded set in the moduli space. On the other hand, by the choice of our generic path in Theorem 3.4.10, the moduli space at $t=\tau_{\infty}$ admits at most finitely many non-regular, embedded disks. Therefore, all disks in $\mathcal{B}$, except at most a finite number of disks, are regular. Consequently, the evaluation map is continuous everywhere in $\mathcal{B}$ and differentiable except at most a finite number of points (leafs).

Moreover, $\mathcal{U}_{\psi\left(\tau_{\infty}, \cdot\right)}$ is an open dense, and irreducible subset of $\mathcal{B}$. If $\tau_{\infty} \neq \bar{t}_{k},(1 \leq k \leq l)$, then all disks in $\mathcal{B}$ are regular and the set of disks which are neither super regular nor almost 
super regular has codimension at least 2. In this case, $\mathcal{B}=\mathcal{F}_{\psi\left(\tau_{\infty}, \cdot\right)}$ is an almost super regular foliation. On the other hand, if $\tau_{\infty}=\bar{t}_{k}$ for some $k$, then either $\mathcal{B}$ contains a finite number of singular disks, or all disks in $\mathcal{B}$ are regular where the codimension for non-almost super regular or non-super regular disks may be 1 . In the case that $\mathcal{B}$ contains a finite number of isolated disks, the codimension of non super regular disks or non almost super regular disks must have codimension 2 or higher. In this case, $\mathcal{B}$ defines an almost super regular foliation. The last remaining case is that $\mathcal{B}$ is regular but the the set of non-super regular or non-almost super regular disks may have codimension 1. In this case, we can perturb this component $\mathcal{B}$ for $t-\bar{t}>0$ small. Following Proposition 3.4.6 the connected component after perturbation defines an almost super regular foliation for $t>\bar{t}$.

In all cases, it is easy to see that $\phi_{\infty}$ is smooth in an open dense subset $\mathcal{R}_{\phi_{\infty}}$ of $\Sigma \times M$. Moreover, we can show that $\mathcal{B}$ is unique since the corresponding partially smooth solution in the limit is unique. This in particular implies that the limit $\mathcal{F}_{\psi\left(\tau_{\infty},,^{\circ}\right)}$ is independent of the time sequence $\tau_{i} \rightarrow \infty$.

Now we return to prove our main theorem.

Proof. To prove openness, we assume $\mathcal{F}_{\psi(\bar{t}, \cdot)}$ is an almost super regular foliation. Here we follow the notations in Theorem 3.4.10. Without loss of generality, we may assume $\bar{t} \leq \bar{t}_{1}$. If $\bar{t}<\bar{t}_{1}$, then $\mathcal{M}_{\psi(\bar{t}, \cdot)}$ is smooth. In particular, the connected component $\overline{\mathcal{U}}_{\phi(\bar{t}, \cdot)}$ is smooth without boundary. Following from the standard deformation theory, this component will deform to a smooth component $\overline{\mathcal{U}}_{\psi(t, \cdot)}$ of $\mathcal{M}_{\psi(t, \cdot)}$ for $t-\bar{t}>0$ small enough. By Theorem 3.4.8 and 3.4.10] $\overline{\mathcal{U}}_{\psi(t, \cdot)}$ induces an almost super regular foliation, so the openness follows in this case.

Now assume $\bar{t}=\bar{t}_{1}$. We want to show that for $\bar{t}_{1}=\bar{t}<t<\bar{t}_{2}$, there is an almost super regular foliation $\mathcal{F}_{\psi(t, \cdot)}$.

By our choice of the generic path $\psi$, we may assume that there are a finite number of embedded, non-regular disks in $\overline{\mathcal{U}}_{\psi(\bar{t}, \cdot)} \backslash \mathcal{U}_{\psi(\bar{t}, \cdot)}$. Since we are interested in preserving this connected component $\mathcal{U}_{\phi(\bar{t}, \cdot)}$, we want to rule out the possibility of either a "merge in" or "spin off" occuring. In other words, there might be another component of $\mathcal{M}_{\psi(\bar{t}, \cdot)}$ connecting with $\overline{\mathcal{U}}_{\psi(\bar{t}, \cdot)}$ through these isolated singular disks: Two components before $t=\bar{t}$ may merge locally into one smooth connected component after $t=\bar{t}$. The situation can also occur in the reverse order: an open set of the moduli $\mathcal{M}_{\psi(t, \cdot)}$ may pinch off of a "neck $S^{2 n-1}$ " at $t=\bar{t}$ and go on to become two separate components after $t=\bar{t}$, at least locally near this "neck.." We call the first case "merge-in" and the second case "pinching-off". If either one occurs, this "good" component will change after singular disks. The deformation of almost super regular foliations is impossible if either of these phenomenon occur beyond the time when singular disks appear. We will deal 
only with the "merge-in" case here, since the other cases (like "the "pinching-off" case) can be handled in a similar fashion.

Note that the "merge-in" of the moduli spaces occurs only at singular disks. Since there is only finite number of singular disks and "merge in" only occur locally near singular disk, we may assume without loss of generality, there is only one non-regular disk $\bar{f}$ in $\overline{\mathcal{U}}_{\psi(t)}$.

Without loss of generality, set $\bar{t}=\bar{t}_{1}$ and $\mathcal{M}_{t}=\mathcal{M}_{\psi(t, 0)}$ is an almost super regular foliation for any $t \in(0, \bar{t}]$. Suppose $\bar{f}$ is the only isolated singular disk at $t=\bar{t}$. Then the metric ball $B_{r}(\bar{f})$ in $\mathcal{M}_{\bar{t}}$ can be represented by a cone in $R^{2 n+1}$ :

$$
\sum_{i=1}^{k} x_{i}^{2}-\sum_{i=k+1}^{2 n+1} x_{i}^{2}=0
$$

where

$$
\sum_{i=k+1}^{2 n+1} x_{i}^{2}<r^{2}
$$

Here $(0,0, \cdots, 0)$ represent $\bar{f}$. The "merge in" or "spin off" case corresponds to $k=2 n$ or $k=1$. We diskuss the "merge in" case here. For $\bar{t}-t>0$ small, the corresponding metric ball in $\mathcal{M}_{t}$ is

$$
\sum_{i=1}^{2 n} x_{i}^{2}-x_{2 n+1}^{2}=t-\bar{t}, \quad x_{2 n+1}^{2}<r^{2}+\bar{t}-t, \quad x_{2 n+1}>0 .
$$

For $t-\bar{t}>0$ small, the corresponding metric ball in $\mathcal{M}_{t}$ is

$$
\sum_{i=1}^{2 n} x_{i}^{2}-x_{2 n+1}^{2}=t-\bar{t}, \quad \sum_{k=1}^{2 n} x_{k}^{2} \leq r^{2}+t-\bar{t} .
$$

Choose a continuous path of disks $f(t) \in \mathcal{M}_{\psi(t, \cdot)}$ such that $f(t)=\bar{f}$ and $f(t)(t \neq \bar{t})$ is either super regular or almost super regular disk. For notational simplicity, we denote $f(t)$ by $\bar{f}$. Note that for $r>0$ small enough, the intersection $B_{r}(\bar{f}) \cap \mathcal{M}_{t}$ consists of two disjoint disks for $t \leq \bar{t}$, but is cylinder-like for $t>\bar{t}$. We consider the intersection of this ball with the central fibre $\{0\} \times M$. In this proof, we use $e v$ to denote the map $\pi \circ e v(0, \cdot)$. Set $e v(\bar{f})=p$.

Note that for $t>\bar{t}$, the boundary of $B_{r}(\bar{f})$ is made of two components $N_{1} \approx N_{2} \approx S^{2 n-1}$ $\left(\approx\right.$ means diffeomorphic to) which are homotopic to each other in $B_{r}(\bar{f})$. These boundary spheres are perturbations of $\partial B_{r}(\bar{f}) \cap \mathcal{M}_{\psi(\bar{t}, \cdot)}$. Pick up one of these spheres, say $N_{1}$ for $t>\bar{t}$. Since each component of $e v\left(B_{r}(\bar{f}) \cap \mathcal{M}_{\psi(\bar{t}, \cdot)}\right)$ bounds a deformation retractable domain in the central fibre $\{0\} \times M$. By continuity, $e v\left(N_{1}\right)$ also bounds a domain $\Omega$ which is deformation contractible to an interior point $q \in \Omega$ for $t-\bar{t}$ sufficiently small. Let us denote this contraction 
by $F:[0,1] \times e v\left(N_{1}\right) \mapsto \Omega$ such that $F(0, p)=p$ and $F(1, p)=q$ for any $p \in e v\left(N_{1}\right)$. Since the set of disks which are neither super regular nor almost super regular has codimension 2 or higher, there is an open subset $V \subset e v\left(N_{1}\right)$ such that $F([0,1) \times V)$ does not intersect with the image of the set of disks which are neither super regular nor almost super regular. Now we can lift this $F([0,1) \times V)$ to $\mathcal{M}_{t}$ since any point in the subset has its pre-image covered by either an super regular disks or an almost super regular disk. This implies that there is a subset $N_{3}$ such that $e v\left(N_{3}\right)$ is a single point, where $N_{3}$ consists of all limiting points of the lifting of $[0,1) \times V$. Clearly, any disk in $N_{3}$ is neither super regular nor almost super regular. Observing that $N_{3}$ has codimension one, we get a contradiction to the fact that the set of all disks which are not almost super regular has codimension at least two.

By similar arguments, we can prove that there is no "pinching-off" at $t=\bar{t}$.

\section{Basic curvature equations along leaves}

\subsection{Introduction}

In this section, we show some deformation results for solutions of the homogenous complex Monge Ampere equations. In particular, we give a basic curvature formula for the restriction of involved metric to leaves. This formula plays a crucial role in deriving key a priori estimate. Suppose that $\phi$ is a solution of (1.1). Suppose that the $\pi_{2}^{*} \omega+\sqrt{-1} \partial \bar{\partial} \phi$, referred as the Levi form, has constant co-rank 1 . This gives rise to a foliation of the domain by holomorphic disks. We further assume that:

\section{At each point of the domain, the leaf vector field is always transversal to a $M$.}

Under this assumption, the Levi form restricts to a Kähler metric in $M$ for each $z \in \Sigma$. In this way, a solution of (1.1) can be alternatively viewed as a disk family of Kähler metrics satisfying certain geometric conditions. To understand the geometry of this family of Kähler metrics, we will study the restriction of the complex tangent bundle $T M$ over this family of holomorphic disks. These bundles are equipped with natural Hermitian metrics, so we obtain a family of Hermitian bundle over disks. In this section, we will compute curvature of these Hermitian bundles. The main results are

1. The curvature of these Hermitian metrics is always non-positive (Theorem 4.2.8);

2. The foliation is holomorphic if and only if the "trace of the curvature" of these Hermitian metrics vanishes (Theorem 4.2.9);

3. The trace of the Hermitian curvature is always super harmonic (Corollary 4.2.11).

The results in this section lay foundations for the global deformation of almost super regular foliations in this paper. 


\subsection{Hermitian Curvature formulas}

In local coordinate, we write

$$
\omega_{0}=\sqrt{-1} \sum_{\alpha, \beta=1}^{n} g_{0, \alpha \bar{\beta}} d w^{\alpha} \wedge d w^{\bar{\beta}}, \quad \omega_{\phi}=\sqrt{-1} \sum_{\alpha, \beta=1}^{n} g_{\phi, \alpha \bar{\beta}} d w^{\alpha} \wedge d w^{\bar{\beta}}
$$

where

$$
g_{\alpha \bar{\beta}}=g_{0, \alpha \bar{\beta}}+\frac{\partial^{2} \phi}{\partial w_{\alpha} \partial \bar{w}_{\beta}}, \quad \forall \alpha, \beta=1,2 \cdots n .
$$

As before, $z$ denotes the coordinate variable of $\Sigma$. Then (1.1) can be re-written as

$$
\frac{\partial^{2} \phi}{\partial z \partial \bar{z}}-g_{\phi}^{\alpha \bar{\beta}} \frac{\partial^{2} \phi}{\partial z \partial \bar{w}_{\beta}} \frac{\partial^{2} \phi}{\partial \bar{z} \partial w_{\alpha}}=0
$$

Here we are assuming that $\omega_{\phi}=\omega_{0}+\sqrt{-1} \partial \bar{\partial} \phi>0$ in $M$. In this section, we will simply write $g$ for the metric $g_{\phi}$ if there is no confusion. Write the leaf vector field (cf. Definition 1.4.3) as

$$
X=\sum_{\alpha=1}^{n} \eta^{\alpha} \frac{\partial}{\partial w_{\alpha}}=\sum_{\alpha=1}^{n}-g^{\alpha \bar{\beta}} \frac{\partial^{2} \phi}{\partial z \partial \bar{w}_{\beta}} \frac{\partial}{\partial w_{\alpha}} .
$$

Denote the linearized operator by $\Delta_{z}$. There is a natural splitting of this operator since all disks are holomorphic:

$$
\Delta_{z}=\partial_{z} \bar{\partial}_{z}, \quad \text { where } \partial_{z}=\frac{\partial}{\partial z}+\eta^{\alpha} \frac{\partial}{\partial w_{\alpha}} .
$$

Proposition 4.2.1. The leaf vector field $X$ (cf. Defi. 1.3) is holomorphic in $z$. In other words

$$
\left[\partial_{z}, \overline{\partial_{z}}\right]=\partial_{\bar{z}} X=0
$$

Proof. Direct calculation.

Proposition 4.2.2. The commutator of local differtial operator on $T M$ and the leaf derivative $\partial_{z}$ is

$$
\left[\partial_{z}, \frac{\partial}{\partial w_{i}}\right]=-\frac{\partial \eta^{\alpha}}{\partial w_{i}} \frac{\partial}{\partial w_{\alpha}}, \quad\left[\partial_{z}, \frac{\partial}{\partial \overline{w_{i}}}\right]=-\frac{\partial \eta^{\alpha}}{\partial \overline{w_{i}}} \frac{\partial}{\partial w_{\alpha}} .
$$

Proof.

$$
\left[\partial_{z}, \frac{\partial}{\partial w_{i}}\right]=\left[\frac{\partial}{\partial z}+\eta^{\alpha} \frac{\partial}{\partial w_{\alpha}}, \frac{\partial}{\partial w_{i}}\right]=-\frac{\partial \eta^{\alpha}}{\partial w_{i}} \frac{\partial}{\partial w_{\alpha}},
$$

and

$$
\left[\partial_{z}, \frac{\partial}{\partial \overline{w_{i}}}\right]=\left[\frac{\partial}{\partial z}+\eta^{\alpha} \frac{\partial}{\partial w_{\alpha}}, \frac{\partial}{\partial w_{i}}\right]=-\frac{\partial \eta^{\alpha}}{\partial \overline{w_{i}}} \frac{\partial}{\partial w_{\alpha}} .
$$


Remark 4.2.3. Note that that $\frac{\partial \eta^{\alpha}}{\partial w_{i}}$ is not a globally well defined tensor, while $\frac{\partial \eta^{\alpha}}{\partial \overline{w_{i}}}$ is a globally well defined tensor since

$$
\frac{\partial \eta^{\alpha}}{\partial \bar{w}_{i}}=-g^{\alpha \bar{s}}\left(\frac{\partial \phi}{\partial z}\right)_{, \bar{i} \bar{s}} .
$$

In a local coordinate chart, suppose $g_{0, \alpha \bar{\beta}}=\frac{\partial^{2} \rho}{\partial w_{\alpha} \overline{\partial w_{\beta}}}$ where $\rho$ is independent of the $z$ direction. Let $\Phi$ denote the local Kähler potenital for $g$, then

$$
\Phi=\phi+\rho
$$

Proposition 4.2.4. For global Kähler distortion potentials, the following is true:

$$
\Delta_{z} \phi=\partial_{z} \bar{\partial}_{z}(\phi)=-|X|_{g_{0}}^{2}
$$

Proof. By a straightforward calculation.

Lemma 4.2.5. (The Bootstrapping lemma): The following commutation formula for the third transversal derivatives holds

$$
\frac{\partial \eta^{\alpha}}{\partial w_{i}}=-g^{\alpha \bar{\beta}} \partial_{z} g_{i \bar{\beta}}, \quad \text { and } \quad \frac{\partial \eta^{\alpha}}{\partial \bar{w}_{i}}=-g^{\alpha \bar{\beta}} \partial_{z} \frac{\partial^{2} \Phi}{\partial \bar{w}_{i} \partial \bar{w}_{\beta}} .
$$

Proof. In local coordinate, we have

$$
\begin{aligned}
\partial_{z} g_{i \bar{\beta}} & =\partial_{z} \frac{\partial^{2} \Phi}{\partial w_{i} \partial \bar{w}_{\beta}}=\frac{\partial}{\partial w_{i}} \partial_{z} \frac{\partial \Phi}{\partial \bar{w}_{\beta}}-\frac{\partial \eta^{\alpha}}{\partial w_{i}} \frac{\partial \Phi}{\partial \bar{w}_{\beta} \partial w_{\alpha}} \\
& =-\frac{\partial \eta^{\alpha}}{\partial w_{i}} g_{\alpha \bar{\beta}} .
\end{aligned}
$$

On the other hand,

$$
\begin{aligned}
\partial_{z} \frac{\partial^{2} \Phi}{\partial w_{i} \partial w_{\beta}} & =\frac{\partial}{\partial w_{i}} \partial_{z} \frac{\partial \Phi}{\partial w_{\beta}}-\frac{\partial \eta^{\alpha}}{\partial w_{i}} \frac{\partial \Phi}{\partial w_{\beta} \partial w_{\alpha}} \\
& =-\frac{\partial \eta^{\alpha}}{\partial w_{i}} g_{\alpha \bar{\beta}} .
\end{aligned}
$$

Remark 4.2.6. The significance of this equation (4.7) is that it changes the 1st derivatives on the transversal direction into the 1st derivatives along the disk of the 2nd order jet of local Kähler potentials.

Lemma 4.2.7. (Regularity lemma). The following equation holds along the disk (for any $\alpha, i=$ $1,2, \cdots n)$ :

$$
\overline{\partial_{z}} \frac{\partial \eta^{\alpha}}{\partial w_{i}}=-\overline{\left(\frac{\partial \eta^{\beta}}{\overline{\partial w_{i}}}\right)} \frac{\partial \eta^{\alpha}}{\overline{\partial w_{\beta}}}, \quad \text { and } \quad \overline{\partial_{z}} \frac{\partial \eta^{\alpha}}{\overline{\partial w_{i}}}=-\overline{\left(\frac{\partial \eta^{\beta}}{\partial w_{i}}\right)} \frac{\partial \eta^{\alpha}}{\overline{\partial w_{\beta}}}
$$


Proof. This lemma follow from the commutation formula 4.5) and Lemma 4.2.5 immediately.

The major obstacle in establishing an a priori estimate for (1.1) is that its linearized operator $\Delta_{z}$ only has rank 1. This is a severe restriction in deriving any meaningful estimate directly. However, we may restrict the $k-(k=0,1,2 \cdots)$ th jet of the potential function $\Phi$ along the disk and study the geometric equation it must satisfy. Notice that the restriction of $T M$ bundle to the disk is a trivial $C^{n}$ bundle. At each point, $g_{\phi}$ is a Hermitian metric in this $T M$ bundle over disk. Suppose that $\left(F_{\beta}^{\alpha}\right)$ is the Hermitian curvature of this Hermitian metric.

Theorem 4.2.8. The curvature of the bundle metric is always non-positive.

Proof.

$$
\begin{aligned}
F_{\alpha}^{r} & =-\partial_{\bar{z}}\left(g^{r \bar{\delta}} \partial_{z} g_{\phi, \alpha \bar{\delta}}\right) \\
& =\partial_{\bar{z}} \frac{\partial \eta^{r}}{\partial w_{\bar{\alpha}}}=-\frac{\partial \eta^{r}}{\partial w_{\bar{i}}} \cdot \frac{\partial \eta^{\bar{i}}}{\partial w_{\alpha}} .
\end{aligned}
$$

It is not difficult to see that the last term in the right hand side is a Hermitian Symmetric non-positive 2-tensors. For any holomorphic section $s: \Sigma \rightarrow T^{1,0} M$, we have

$$
\begin{aligned}
F(s, s) & =F_{\beta}^{\alpha} s^{\beta} s^{\bar{\gamma}} g_{\phi_{\alpha \bar{\gamma}}} \\
& =-\frac{\partial \eta^{\alpha}}{\partial w_{\bar{i}}} \cdot \frac{\partial \eta^{\bar{i}}}{\partial w_{\beta}} s^{\beta} s^{\bar{\gamma}} g_{\phi_{\alpha \bar{\gamma}}} \\
& =-g^{\alpha \bar{b}} g^{\bar{i} a}\left(\frac{\partial \varphi}{\partial z}\right)_{, \bar{i} \bar{b}} \cdot\left(\frac{\partial \varphi}{\partial \bar{z}}\right)_{, \beta a} s^{\beta} s^{\bar{\gamma}} g_{\alpha \bar{\gamma}} \\
& =-g^{\bar{i} a}\left(\frac{\partial \varphi}{\partial z}\right)_{, \bar{i} \bar{r}} \cdot\left(\frac{\partial \varphi}{\partial \bar{z}}\right)_{, \beta a} s^{\beta} s^{\bar{\gamma}} \leq 0 .
\end{aligned}
$$

The last inequality holds since $\phi$ is real valued potential function and the $(2,0)$ or $(0,2)$ part of the Hessian is symmetric.

A quick corollary follows

Theorem 4.2.9. This foliation by holomorphic disks is a holomorphic foliation if and only if the curvature of these Hermitian metrics vanishes.

Proof. This is quite evident from Theorem 4.2.8.

Proposition 4.2.10. $g^{i \bar{j}} \frac{\partial \eta^{\alpha}}{\partial w_{j}}$ is holomorphic along the disk.

Proof.

$$
\overline{\partial_{z}} g^{i \bar{j}}=-g^{i \bar{k}} \overline{\partial_{z}} g_{\bar{k} l} g^{l \bar{j}}=g^{i \bar{k}} \frac{\partial \eta^{\bar{\beta}}}{\overline{\partial w_{k}}} g_{l \bar{\beta}} g^{l \bar{j}}=g^{i \bar{k}} \frac{\partial \eta^{\bar{j}}}{\overline{\partial w_{k}}}
$$


Now, we have

$$
\begin{aligned}
\overline{\partial_{z}}\left(g^{i \bar{j}} \frac{\partial \eta^{\alpha}}{\overline{\partial w_{j}}}\right) & =\left(\overline{\partial_{z}} g^{i \bar{j}}\right) \frac{\partial \eta^{\alpha}}{\partial w_{j}}+g^{i \bar{j}} \overline{\partial_{z}} \frac{\partial \eta^{\alpha}}{\partial w_{j}} \\
& =g^{i \bar{k}} \overline{\frac{\partial \eta^{\bar{j}}}{\partial w_{k}}} \frac{\partial \eta^{\alpha}}{\partial w_{j}}-g^{i \bar{j}} \overline{\left(\frac{\partial \eta^{\beta}}{\partial w_{j}}\right)} \frac{\partial \eta^{\alpha}}{\partial w_{\beta}}=0 .
\end{aligned}
$$

Corollary 4.2.11. ${ }^{18}$. The anti-canonical line bundle in $M$ equipped with $\omega_{\phi}^{n}$ as Hermitian metric, restricted to a holomorphic disk, has non-positive curvature. More precisely, we have

$$
\Delta_{z} \log \omega_{\phi}^{n}=\partial_{z} \overline{\partial_{z}} \log \omega_{\phi}^{n}=\frac{\partial \eta^{\alpha}}{\overline{\partial w_{i}}} \overline{\left(\frac{\partial \eta^{i}}{\partial w_{\alpha}}\right)} .
$$

Note that the right hand side measures whether the given geodesic(or disk version) is holomorphic or not.

Proof. In a local coordinate, we have

$$
\partial_{z} \log \omega_{\phi}^{n}=g^{\alpha \bar{\beta}} \partial_{z} g_{\bar{\beta} \alpha}=-\frac{\partial \eta^{\alpha}}{\partial w_{\alpha}} .
$$

Thus,

$$
\begin{aligned}
\overline{\partial_{z}} \partial_{z} \log \omega_{\phi}^{n} & =-\overline{\partial_{z}} \frac{\partial \eta^{\alpha}}{\partial w_{\alpha}}=-\left(\frac{\partial}{\partial w_{\alpha}} \overline{\partial_{z}}-\frac{\partial \eta^{\bar{\beta}}}{\partial w_{\alpha}} \frac{\partial}{\partial w_{\bar{\beta}}}\right) \eta^{\alpha} \\
& =\frac{\partial \eta^{\bar{\beta}}}{\partial w_{\alpha}} \frac{\partial \eta^{\alpha}}{\partial \bar{w}_{\beta}} \geq 0 .
\end{aligned}
$$

Set $-S=F_{\alpha}^{\alpha}=\frac{\partial \eta^{\bar{\beta}}}{\partial w_{\alpha}} \frac{\partial \eta^{\alpha}}{\partial \bar{w}_{\beta}}=g_{\phi}{ }^{\alpha \bar{p}} \phi_{, z \bar{i} \bar{p}} g_{\phi}{ }^{\bar{i} q} \phi_{, \bar{z} \alpha} \geq 0$ (this denotes the covariant derivatives w.r.t. $\left.g_{\phi}.\right)$ as the trace of the curvature of this $T M$ bundle with Hermitian metric $g_{\phi}$.

Theorem 4.2.12. The trace of the Hermitian curvature satisfies the following

$$
\begin{aligned}
& \partial_{z} \overline{\partial_{z}} S=\left(\partial_{z} \frac{\partial \eta^{\alpha}}{\partial \overline{w_{i}}}-\frac{\partial \eta^{\beta}}{\partial \overline{w_{i}}} \frac{\partial \eta^{\alpha}}{\partial w_{\beta}}\right)\left(\overline{\partial_{z}} \frac{\partial \eta^{\bar{i}}}{\partial w_{\alpha}}-\frac{\partial \eta^{\bar{i}}}{\partial \overline{w_{p}}} \frac{\partial \eta^{\bar{p}}}{\partial w_{\alpha}}\right) \\
&+2 \frac{\partial \eta^{\alpha}}{\partial \bar{w}_{i}} \frac{\partial \eta^{\bar{p}}}{\partial w_{\alpha}} \frac{\partial \eta^{\beta}}{\partial \bar{w}_{p}} \frac{\partial \eta^{\bar{i}}}{\partial w_{\beta}} \\
&=\frac{\partial}{\partial \bar{w}_{i}} \partial_{z}\left(\eta^{\alpha}\right) \frac{\partial}{\partial w_{\alpha}} \overline{\partial_{z}}\left(\eta^{\bar{i}}\right) \\
&+2 g_{\phi}{ }^{\alpha \bar{i}} g_{\phi} \overline{r j} g_{\phi}{ }^{\beta \bar{k}} g_{\phi} \bar{\delta} l \phi_{, z i \bar{i}} \phi_{, \bar{z} j \beta} \phi_{, z \overline{k \delta}} \phi_{, \bar{z} l \alpha} \geq \frac{2}{n} S^{2} .
\end{aligned}
$$

\footnotetext{
${ }^{18}$ This was first derived in 1996 by the first author and S. Donaldson using some different methods.
} 
Proof. According to Lemma 4.2.7 we have

$$
\overline{\partial_{z}} \frac{\partial \eta^{\alpha}}{\partial \bar{w}_{i}}=-\frac{\partial \eta^{\bar{\beta}}}{\partial \overline{w_{i}}} \frac{\partial \eta^{\alpha}}{\partial \overline{w_{\beta}}} .
$$

Thus

$$
\begin{aligned}
\partial_{z} \overline{\partial_{z}} \frac{\partial \eta^{\alpha}}{\partial \bar{w}_{i}} & =-\partial_{z}\left(\frac{\partial \eta^{\bar{\beta}}}{\partial \overline{w_{i}}}\right) \frac{\partial \eta^{\alpha}}{\partial \overline{w_{\beta}}}-\frac{\partial \eta^{\bar{\beta}}}{\partial \overline{w_{i}}} \partial_{z} \frac{\partial \eta^{\alpha}}{\partial \overline{w_{\beta}}} \\
& =\frac{\partial \eta^{p}}{\partial \bar{w}_{i}} \frac{\partial \eta^{\beta}}{\partial w_{p}} \frac{\partial \eta^{\alpha}}{\partial \bar{w}_{\beta}}-\frac{\partial \eta^{\beta}}{\partial \bar{w}_{i}} \partial z \frac{\partial \eta^{\alpha}}{\partial \overline{w_{\beta}}} .
\end{aligned}
$$

We have

$$
\begin{aligned}
& \Delta_{z}\left(\frac{\partial \eta^{\alpha}}{\partial \bar{w}_{i}} \frac{\partial \eta^{\bar{i}}}{\partial w_{\alpha}}\right) \\
& =\partial_{z}\left(\frac{\partial \eta^{\alpha}}{\partial \bar{w}_{i}}\right) \overline{\partial_{z}}\left(\frac{\partial \eta^{\bar{i}}}{\partial w_{\alpha}}\right)+\overline{\partial_{z}}\left(\frac{\partial \eta^{\alpha}}{\partial \bar{w}_{i}}\right) \partial_{z}\left(\frac{\partial \eta^{\bar{i}}}{\partial w_{\alpha}}\right) \\
& +\triangle_{z}\left(\frac{\partial \eta^{\alpha}}{\partial \bar{w}_{i}}\right)\left(\frac{\partial \eta^{\bar{i}}}{\partial w_{\alpha}}\right)+\left(\frac{\partial \eta^{\alpha}}{\partial \bar{w}_{i}}\right) \triangle_{z}\left(\frac{\partial \eta^{\bar{i}}}{\partial w_{\alpha}}\right) \\
& =\partial_{z}\left(\frac{\partial \eta^{\alpha}}{\partial \overline{w_{i}}}\right) \overline{\partial_{z}}\left(\frac{\partial \eta^{\bar{i}}}{\partial w_{\alpha}}\right)+\frac{\partial \eta^{\bar{\beta}}}{\partial \bar{w}_{i}} \frac{\partial \eta^{\alpha}}{\partial \bar{w}_{\beta}} \frac{\partial \eta^{p}}{\partial w_{\alpha}} \frac{\partial \eta^{\bar{i}}}{\partial w_{p}} \\
& +\left(\frac{\partial \eta^{p}}{\partial \bar{w}_{i}} \frac{\partial \eta^{\bar{\beta}}}{\partial w_{p}} \frac{\partial \eta^{\alpha}}{\partial \bar{w}_{\beta}}-\frac{\partial \eta^{\bar{\beta}}}{\partial \bar{w}_{i}} \partial_{z} \frac{\partial \eta^{\alpha}}{\partial \bar{w}_{\beta}}\right) \frac{\partial \eta^{\bar{i}}}{\partial w_{\alpha}} \\
& +\frac{\partial \eta^{\alpha}}{\partial \bar{w}_{i}} \overline{\left(\frac{\partial \eta^{p}}{\partial \bar{w}_{\alpha}} \frac{\partial \eta^{\bar{\beta}}}{\partial w_{p}} \frac{\partial \eta^{i}}{\partial \bar{w}_{\beta}}-\frac{\partial \eta^{\bar{\beta}}}{\partial \bar{w}_{\alpha}} \partial_{z} \frac{\partial \eta^{i}}{\partial \bar{w}_{\beta}}\right)} \\
& =\partial_{z}\left(\frac{\partial \eta^{\alpha}}{\partial \bar{w}_{i}}\right) \overline{\partial_{z}}\left(\frac{\partial \eta^{\bar{i}}}{\partial w_{\alpha}}\right)+\frac{\partial \eta^{\bar{\beta}}}{\partial \bar{w}_{i}} \frac{\partial \eta^{\alpha}}{\partial \bar{w}_{\beta}} \frac{\partial \eta^{p}}{\partial w_{\alpha}} \frac{\partial \eta^{\bar{i}}}{\partial w_{p}}-\partial_{z}\left(\frac{\partial \eta^{\alpha}}{\partial \bar{w}_{\beta}}\right) \frac{\partial \eta^{\bar{\beta}}}{\partial \bar{w}_{i}} \frac{\partial \eta^{\bar{i}}}{\partial w_{\alpha}} \\
& -\frac{\partial \eta^{\alpha}}{\partial \bar{w}_{i}} \frac{\partial \eta^{\beta}}{\partial w_{\alpha}} \overline{\partial_{z}} \frac{\partial \eta^{\bar{i}}}{\partial w_{\beta}}+\frac{\partial \eta^{p}}{\partial \bar{w}_{i}} \frac{\partial \eta^{\bar{\beta}}}{\partial w_{p}} \frac{\partial \eta^{\alpha}}{\partial \bar{w}_{\beta}} \frac{\partial \eta^{\bar{i}}}{\partial w_{\alpha}}+\frac{\partial \eta^{\alpha}}{\partial \bar{w}_{i}} \frac{\partial \eta^{\bar{p}}}{\partial w_{\alpha}} \frac{\partial \eta^{\beta}}{\partial \bar{w}_{p}} \frac{\partial \eta^{\bar{i}}}{\partial w_{\beta}} \\
& =\partial_{z}\left(\frac{\partial \eta^{\alpha}}{\partial \bar{w}_{i}}\right) \overline{\partial_{z}}\left(\frac{\partial \eta^{\bar{i}}}{\partial w_{\alpha}}\right)+\frac{\partial \eta^{\bar{\beta}}}{\partial \bar{w}_{i}} \frac{\partial \eta^{\alpha}}{\partial \bar{w}_{\beta}} \frac{\partial \eta^{p}}{\partial w_{\alpha}} \frac{\partial \eta^{\bar{i}}}{\partial w_{p}}-\partial_{z}\left(\frac{\partial \eta^{\alpha}}{\partial \bar{w}_{i}}\right) \frac{\partial \eta^{\bar{i}}}{\partial \bar{w}_{p}} \frac{\partial \eta^{\bar{p}}}{\partial w_{\alpha}} \\
& -\frac{\partial \eta^{\beta}}{\partial \bar{w}_{i}} \frac{\partial \eta^{\alpha}}{\partial w_{\beta}} \overline{\partial_{z}} \frac{\partial \eta^{\bar{i}}}{\partial w_{\alpha}}+\frac{\partial \eta^{p}}{\partial \bar{w}_{i}} \frac{\partial \eta^{\bar{\beta}}}{\partial w_{p}} \frac{\partial \eta^{\alpha}}{\partial \bar{w}_{\beta}} \frac{\partial \eta^{\bar{i}}}{\partial w_{\alpha}}+\frac{\partial \eta^{\alpha}}{\partial \bar{w}_{i}} \frac{\partial \eta^{\bar{p}}}{\partial w_{\alpha}} \frac{\partial \eta^{\beta}}{\partial \bar{w}_{p}} \frac{\partial \eta^{\bar{i}}}{\partial w_{\beta}} \\
& =\left(\partial_{z} \frac{\partial \eta^{\alpha}}{\partial \overline{w_{i}}}-\frac{\partial \eta^{\beta}}{\partial \bar{w}_{i}} \frac{\partial \eta^{\alpha}}{\partial w_{\beta}}\right)\left(\overline{\partial_{z}} \frac{\partial \eta^{\bar{i}}}{\partial w_{\alpha}}-\frac{\partial \eta^{\bar{i}}}{\partial \bar{w}_{p}} \frac{\partial \eta^{\bar{p}}}{\partial w_{\alpha}}\right)-\frac{\partial \eta^{\beta}}{\partial \overline{w_{i}}} \frac{\partial \eta^{\alpha}}{\partial w_{\beta}} \frac{\partial \eta^{\bar{i}}}{\partial \overline{w_{p}}} \frac{\partial \eta^{\bar{p}}}{\partial w_{\alpha}} \\
& +\frac{\partial \eta^{\bar{\beta}}}{\partial \bar{w}_{i}} \frac{\partial \eta^{\alpha}}{\partial \bar{w}_{\beta}} \frac{\partial \eta^{p}}{\partial w_{\alpha}} \frac{\partial \eta^{\bar{i}}}{\partial w_{p}}+\frac{\partial \eta^{p}}{\partial \bar{w}_{i}} \frac{\partial \eta^{\bar{\beta}}}{\partial w_{p}} \frac{\partial \eta^{\alpha}}{\partial \bar{w}_{\beta}} \frac{\partial \eta^{\bar{i}}}{\partial w_{\alpha}}+\frac{\partial \eta^{\alpha}}{\partial \bar{w}_{i}} \frac{\partial \eta^{\bar{p}}}{\partial w_{\alpha}} \frac{\partial \eta^{\beta}}{\partial \bar{w}_{p}} \frac{\partial \eta^{\bar{i}}}{\partial w_{\beta}} \\
& =\left(\partial_{z} \frac{\partial \eta^{\alpha}}{\partial \bar{w}_{i}}-\frac{\partial \eta^{\beta}}{\partial \bar{w}_{i}} \frac{\partial \eta^{\alpha}}{\partial w_{\beta}}\right)\left(\overline{\partial_{z}} \frac{\partial \eta^{\bar{\eta}}}{\partial w_{\alpha}}-\frac{\partial \eta^{\bar{i}}}{\partial \bar{w}_{p}} \frac{\partial \eta^{\bar{p}}}{\partial w_{\alpha}}\right)-\frac{\partial \eta^{\beta}}{\partial \bar{w}_{i}} \frac{\partial \eta^{\alpha}}{\partial w_{\beta}} \frac{\partial \eta^{\bar{p}}}{\partial w_{\alpha}} \frac{\partial \eta^{\bar{i}}}{\partial \bar{w}_{p}} \\
& +\frac{\partial \eta^{\alpha}}{\partial \bar{w}_{\beta}} \frac{\partial \eta^{p}}{\partial w_{\alpha}} \frac{\partial \eta^{\bar{i}}}{\partial w_{p}} \frac{\partial \eta^{\bar{\beta}}}{\partial \bar{w}_{i}}+\frac{\partial \eta^{p}}{\partial \bar{w}_{i}} \frac{\partial \eta^{\bar{\beta}}}{\partial w_{p}} \frac{\partial \eta^{\alpha}}{\partial \bar{w}_{\beta}} \frac{\partial \eta^{\bar{i}}}{\partial w_{\alpha}}+\frac{\partial \eta^{\alpha}}{\partial \bar{w}_{i}} \frac{\partial \eta^{\bar{p}}}{\partial w_{\alpha}} \frac{\partial \eta^{\beta}}{\partial \bar{w}_{p}} \frac{\partial \eta^{\bar{i}}}{\partial w_{\beta}} \\
& =\left(\partial_{z} \frac{\partial \eta^{\alpha}}{\partial \bar{w}_{i}}-\frac{\partial \eta^{\beta}}{\partial \bar{w}_{i}} \frac{\partial \eta^{\alpha}}{\partial w_{\beta}}\right)\left(\overline{\partial_{z}} \frac{\partial \eta^{\bar{i}}}{\partial w_{\alpha}}-\frac{\partial \eta^{\bar{i}}}{\partial \bar{w}_{p}} \frac{\partial \eta^{\bar{p}}}{\partial w_{\alpha}}\right)+2 \frac{\partial \eta^{\alpha}}{\partial \bar{w}_{i}} \frac{\partial \eta^{\bar{p}}}{\partial w_{\alpha}} \frac{\partial \eta^{\beta}}{\partial \bar{w}_{p}} \frac{\partial \eta^{\bar{i}}}{\partial w_{\beta}} .
\end{aligned}
$$

The last equality holds because in the line above the last equation, the 2rd term and the 3rd cancel each other, while the $3 r d$ and 4 th term are the same.

Note that $\partial_{z} \frac{\partial \eta^{\alpha}}{\partial \bar{w}_{i}}-\frac{\partial \eta^{\beta}}{\partial \bar{w}_{i}} \frac{\partial \eta^{\alpha}}{\partial w_{\beta}}$ is a tensor since

$$
\begin{aligned}
\partial_{z} \frac{\partial \eta^{\alpha}}{\partial \bar{w}_{i}}-\frac{\partial \eta^{\beta}}{\partial \bar{w}_{i}} \frac{\partial \eta^{\alpha}}{\partial w_{\beta}} & =\frac{\partial}{\partial z} \frac{\partial \eta^{\alpha}}{\partial \bar{w}_{i}}+\eta^{r} \frac{\partial}{\partial w_{r}} \frac{\partial \eta^{\alpha}}{\partial \bar{w}_{i}}-\frac{\partial \eta^{\beta}}{\partial \overline{w_{i}}}\left(\frac{\partial \eta^{\alpha}}{\partial w_{\beta}}+\eta^{r} \Gamma_{r \beta}^{\alpha}-\eta^{r} \Gamma_{r \beta}^{\alpha}\right) \\
& =\frac{\partial}{\partial z} \frac{\partial \eta^{\alpha}}{\partial \bar{w}_{i}}+\eta^{r}\left(\frac{\partial}{\partial w_{r}} \frac{\partial \eta^{\alpha}}{\partial \overline{w i}_{i}}+\frac{\partial \eta^{\beta}}{\partial \bar{w}_{i}} \Gamma_{r \beta}^{\alpha}\right)-\frac{\partial \eta^{\beta}}{\partial \bar{w}_{i}} \eta_{, \beta}^{\alpha} \\
& =\frac{\partial}{\partial z} \frac{\partial \eta^{\alpha}}{\partial \bar{w}_{i}}+\eta^{r} \eta_{, i r}^{\alpha}-\frac{\partial \eta^{\beta}}{\partial \bar{w}_{i}} \eta_{, \beta}^{\alpha} .
\end{aligned}
$$


Moreover, this is a $(0,2)$ symmetric tensor since

$$
\begin{aligned}
\partial_{z} \frac{\partial \eta^{\alpha}}{\partial \bar{w}_{i}}-\frac{\partial \eta^{\beta}}{\partial \bar{w}_{i}} \frac{\partial \eta^{\alpha}}{\partial w_{\beta}} & =\partial_{z}\left(-g^{\alpha \bar{\beta}} \partial_{z} \frac{\partial^{2} \Phi}{\partial w_{i} \partial w_{\beta}}\right)-\frac{\partial \eta^{\beta}}{\partial \bar{w}_{i}} \frac{\partial \eta^{\alpha}}{\partial w_{\beta}} \\
& =g^{\alpha \bar{p}} \partial_{z} g_{\bar{p} q} g^{q \bar{\beta}} \frac{\partial^{2} \Phi}{\partial w_{i} \partial w_{\beta}}-g^{\alpha \bar{\beta}}\left(\partial_{z}\right)^{2} \frac{\partial^{2} \Phi}{\partial w_{i} \partial w_{\beta}}-\frac{\partial \eta^{\beta}}{\partial \bar{w}_{i}} \frac{\partial \eta^{\alpha}}{\partial w_{\beta}} \\
& =\frac{\partial \eta^{q}}{\partial \bar{w}_{i}} \frac{\partial \eta^{\alpha}}{\partial w_{q}}-g^{\alpha \bar{\beta}}\left(\partial_{z}\right)^{2} \frac{\partial^{2} \Phi}{\partial w_{i} \partial w_{\beta}}-\frac{\partial \eta^{\beta}}{\partial \bar{w}_{i}} \frac{\partial \eta^{\alpha}}{\partial w_{\beta}} \\
& =-g^{\alpha \bar{\beta}}\left(\partial_{z}\right)^{2} \frac{\partial^{2} \Phi}{\partial w_{i} \partial w_{\beta}} \\
& =-g^{\alpha \bar{\beta}} \partial_{z}\left(\frac{\partial \phi}{\partial z}\right)_{, \bar{i} \bar{\beta}} .
\end{aligned}
$$

Thus, the first term in the equation (4.10) can be changed into

$$
\begin{aligned}
\left(\partial_{z} \frac{\partial \eta^{\alpha}}{\partial \bar{w}_{i}}-\right. & \left.\frac{\partial \eta^{\beta}}{\partial \bar{w}_{i}} \frac{\partial \eta^{\alpha}}{\partial w_{\beta}}\right)\left(\overline{\partial_{z}} \frac{\partial \eta^{\bar{i}}}{\partial w_{\alpha}}-\frac{\partial \eta^{\bar{i}}}{\partial \bar{w}_{p}} \frac{\partial \eta^{\bar{p}}}{\partial w_{\alpha}}\right) \\
& =g^{\alpha \bar{\beta}}\left(\partial_{z}\right)^{2} \frac{\partial^{2} \Phi}{\partial w_{i} \partial w_{\beta}} g^{\overline{i p}}\left(\overline{\partial_{z}}\right)^{2} \frac{\partial^{2} \Phi}{\partial w_{\alpha} \partial w_{p}} \geq 0
\end{aligned}
$$

The lemma is then proved.

This theorem should be compared with Calabi's third derivative estimate for the nondegenerate Monge-Ampere equation. Following a result of R. Osserman [23] (later generalized by E. Calabi[3]), we have

Proposition 4.2.13. Let d denote the Euclidean distance to the boundary of $\Sigma$. Then, there exists a uniform constant $C$ such that the trace of the curvature has the following interior estimate

$$
S(z, x)=-\sum_{\alpha=1}^{n} F_{\alpha}^{\alpha} \leq \frac{C}{d(z, \partial \Sigma)^{2}} .
$$

This estimate plays a crucial in obtaining a priori estimate for super regular discs.

\section{Compactness of holomorphic disks}

In this section, we continue our study of the HCMA equation from the point of view initiated in the previous section. Namely, in the foliation by holomorphic disks of $\Sigma \times M$, we study the family of restricted $T M$ bundles equipped with the varying Hermitian metric $g_{\phi}$. We introduce two geometrical quantities which can be used to study the compactness problem of holomorphic disks. For any $\phi_{0}: \partial \Sigma \rightarrow \mathcal{H}$ and any holomorphic disk $f \in \mathcal{M}_{\phi_{0}}$, we define its area as

$$
A(f)=\int_{\Sigma}\left(\frac{\sqrt{-1}}{2} d z \wedge d \bar{z}+f^{*} \omega_{0}\right) .
$$


Note that this is the area of $\pi \circ f(\Sigma)$ in $\Sigma \times M$, not the area of $f(\Sigma) \in \Sigma \times \mathcal{W}_{M}$. When no confusion is possible, we will not distinguish between $f, \pi \circ f$, or even $\pi_{2} \circ \pi \circ f$. Similarly, we also define the Capacity for any super regular disk $f$ in $\mathcal{M}_{\phi_{0}}$ by

$$
\operatorname{Cap}(f)=\int_{\Sigma} \frac{f^{*} \omega_{\phi_{0}\left(z_{0}\right)}^{n}}{f^{*} \omega_{\phi}^{n}} \frac{\sqrt{-1}}{2} d z d \bar{z}
$$

for some $z_{0} \in \partial \Sigma$. For simplicity, we fix $z_{0}$ in this section and assume without loss of generality that $\phi_{0}\left(z_{0}, \cdot\right)=0$. Under this assumption, $\omega_{\phi_{0}\left(z_{0}, \cdot\right)}=\omega_{0}$. Obviously, a non-super regular disk has infinite capacity.

Let us set up some notations first. We will fix a positive number $\alpha \in(0,1)$ in this section. For any $\epsilon, \delta, \Lambda$, let $\phi_{0}$ be any map from $\partial \Sigma$ to $\mathcal{H}$ which satisfies

$$
\omega_{\phi_{0}} \geq \delta \omega_{0}, \quad\left\|\phi_{0}\right\|_{C^{2, \alpha}(\partial \Sigma \times M)} \leq \Lambda .
$$

We further define $\mathcal{C}(\delta, \Lambda)$ as the space of all embedded holomorphic disks in $\Sigma \times \mathcal{W}_{M}$ with vanishing normal Maslov indice, whose boundary lies in $\bar{\Lambda}_{\phi_{0}}$. The space $\mathcal{C}\left(\delta, \Lambda, L_{0}\right)$ is a subset of $\mathcal{C}(\delta, \Lambda)$ such that for each disk $f \in \mathcal{C}\left(\delta, \Lambda, L_{0}\right)$, the corresponding compatible solution $\phi$ of HCMA (1.1) is smooth locally and

$$
|\phi|_{C^{1,1}} \leq L_{0}
$$

hold in a small neighborhood of $f(\Sigma)$. Define

$$
\mathcal{C}\left(\delta, \Lambda, L_{0}, L_{1}\right)=\left\{f \in \mathcal{C}\left(\delta, \Lambda, L_{0}\right) \mid A(f) \leq L_{1}\right\}
$$

and

$$
\mathcal{C}\left(\delta, \Lambda, L_{0}, L_{1}, L_{2}\right)=\left\{f \in \mathcal{C}\left(\delta, \Lambda, L_{0}\right) \mid A(f) \leq L_{1}, \operatorname{Cap}(f) \leq L_{2}\right\} .
$$

In this Section, we will prove

Theorem 5.0.14. The space $\mathcal{C}\left(\delta, \Lambda, L_{0}, L_{1}\right)$ is compact in $\mathcal{C}\left(\delta, \Lambda, L_{0}\right)$.

Theorem 5.0.15. The space $\mathcal{C}\left(\delta, \Lambda, L_{0}, L_{1}, L_{2}\right)$ is compact.

Suppose $\mathcal{F}_{\phi_{0}}$ is an almost super regular foliation, we want to identify $\mathcal{U}_{\phi_{0}}$ with an open and dense subset of $M$ via evaluation map:

$$
\begin{aligned}
& \sharp: \quad \Sigma \times \mathcal{M}_{\phi,} \rightarrow \Sigma \times M \\
& (z, f) \rightarrow \pi \circ \mathrm{ev}(z, f)
\end{aligned}
$$

Then, $\sharp$ is invertible on $\Sigma \times \mathcal{U}_{\phi_{0}}$. Define $F=\pi \circ e v \circ \sharp^{-1}$. We can identify $\mathcal{U}_{\phi_{0}}$ with some open dense subset $\pi \circ \operatorname{ev}\left(z_{0}, \mathcal{U}_{\phi_{0}}\right)$ of $M$. We will use this point of view from time to time in this section. 
Theorem 5.0.16. If $\phi_{0}$ satisfies inequality (5.3) and if $\mathcal{F}_{\phi_{0}}$ is an almost super regular foliation, then there exist two constants $L_{0}, L_{1}$ which depend only on $\delta, \Lambda$ such that

$$
\mathcal{U}_{\phi_{0}} \subset \mathcal{C}\left(\delta, \Lambda, L_{0}, L_{1}\right)
$$

Moreover,

$$
\int_{f \in \mathcal{U}_{\phi_{0}}} \operatorname{Cap}(f) \omega_{\phi_{0}\left(z_{0}, \cdot\right)}^{n}=\int_{f \in \mathcal{U}_{\phi_{0}}} \operatorname{Cap}(f) \omega_{0}^{n} \leq C .
$$

These three theorems can be used to derive a compactness theorem of sequences of almost super regular foliations.

Theorem 5.0.17. Suppose $\left\{\phi_{0}{ }^{(m)}, m \in \mathcal{N}\right\}$ is a sequence of functions in $C^{\infty}(\partial \Sigma, \mathcal{H})$ which satisfies the uniform bound of (5.3) and converges to $\phi_{0}{ }^{(\infty)} \in C^{\infty}(\partial \Sigma, \mathcal{H})$ in the $C^{2, \alpha}(\partial \Sigma \times M)$ norm. Suppose that $\left\{\mathcal{F}_{\phi_{0}^{(m)}}, m \in \mathcal{N}\right\}$ is a sequence of almost super regular foliations, while $\left\{\phi^{(m)}\right\}$ is the corresponding sequence of almost smooth solutions with Drichelet boundary value $\left\{\phi_{0}{ }^{(m)}\right\}$. Passing to a subsequence if necessary, $\mathcal{F}_{\phi_{0}(m)}$ converges to a partially smooth foliation $\mathcal{F}_{\phi_{0}}(\infty)$. In particular, at least one component of $\mathcal{M}_{\phi_{0}(\infty)}$ contains at least one super regular disk. Moreover, $\phi^{(m)}$ converges in the weak $C^{1,1}$ norm to $\phi^{(\infty)}$ such that $\omega_{\phi^{(\infty)}}^{n}$ is a continuous volume form on $\Sigma^{0} \times M$.

\subsection{Proof of Theorem $[5.0 .16$}

Proof. Suppose that $\phi$ is the corresponding almost smooth solution of the HCMA equation 1.1 with the prescribed boundary map $\phi_{0}: \partial \Sigma \rightarrow \mathcal{H}$. By Theroem 1.2 [8], there is a uniform constant $C(\delta, \Lambda)$ such that

$$
|\partial \bar{\partial} \phi| \leq C(\delta, \Lambda)
$$

It is clear that for any super regular disk $f \in \mathcal{U}_{\phi_{0}}$, any local compatible solution to HCMA equation (1.1) must agree with $\phi$ in any small open and saturated neighborhood of $\pi \circ f(\Sigma) \subset$ $\Sigma \times M$. Next, we want to show that each disk must also have a uniform upper bound on its area.

Lemma 5.1.1. For any regular disk $f:(\Sigma, \partial \Sigma) \rightarrow\left(\Sigma \times \mathcal{W}_{M}, \bar{\Lambda}_{\phi_{0}}\right)$ where $\phi$ is the corresponding "almost smooth" solution of equation [1.1. There is a uniform constant $L_{1}$ which depends only on $\delta, \Lambda$ such that $A(f) \leq L_{1}$ holds uniformly.

Proof. Recalled that the leaf vector field $X$ (cf. eq. (4.2) ) along this disk in $\Sigma \times M$ can be expressed as

$$
X=\sum_{\alpha=1}^{n} \eta^{\alpha} \frac{\partial}{\partial w_{\alpha}}=-\sum_{\alpha=1}^{n} g_{\phi}^{\alpha \bar{\beta}} \frac{\partial^{2} \phi}{\partial z \partial w_{\bar{\beta}}} \frac{\partial}{\partial w_{\alpha}} .
$$


According to Corollary 4.2.4, we have

$$
\frac{\partial}{\partial z} \frac{\partial}{\partial \bar{z}}(\phi \circ f)=-g_{0} \bar{\beta} \eta^{\alpha} \eta^{\bar{\beta}}
$$

Using this holomorphic map $f$, the pull back of the fixed product metric metric on $\Sigma \times M$ to $\Sigma$ is:

$$
\begin{aligned}
f^{*}\left(g_{0}+|d z|^{2}\right) & =\left|\frac{\partial}{\partial z}+X\right|_{g_{0}}^{2}|d z|^{2} \\
& =\left(1+g_{0} \bar{\beta} \eta^{\alpha} \eta^{\bar{\beta}}\right)|d z|^{2} .
\end{aligned}
$$

Thus, the area of any disk is :

$$
\begin{aligned}
\int_{f(\Sigma)} 1 & =\int_{\Sigma} f^{*}\left(g_{0}+|d z|^{2}\right) \\
& =\int_{\Sigma}\left(1+g_{0} \overline{\alpha \beta} \eta^{\alpha} \eta^{\bar{\beta}}\right)|d z|^{2} \\
& =|\Sigma|-\int_{\Sigma} \partial_{z} \partial_{\bar{z}} \phi|d z|^{2} \\
& =|\Sigma|-\int_{\Sigma} \frac{\partial^{2}}{\partial z \partial \bar{z}}(\phi \circ f)|d z|^{2} \\
& =|\Sigma|+\int_{\partial \Sigma} \frac{\partial}{\partial z}(\phi \circ f) \cdot \mathbf{n}_{\Sigma} \\
& =|\Sigma|+\left.\int_{\partial \Sigma} \partial_{z} \phi\left|\partial \Sigma \cdot \mathbf{n}_{\Sigma}\right| d z\right|^{2} \\
& =|\tilde{\Sigma}|+\left.\int_{\partial \Sigma}\left(\frac{\partial}{\partial z}+\eta^{\alpha} \frac{\partial}{\partial w_{\alpha}}\right) \phi\left|\partial \Sigma \cdot \mathbf{n}_{\Sigma}\right| d z\right|^{2} \\
& =|\Sigma|+\left.\int_{\partial \Sigma}\left(\frac{\partial \phi}{\partial z}-g_{\phi}^{\alpha \bar{\beta}} \frac{\partial^{2} \phi}{\partial z \partial w_{\bar{\beta}}} \frac{\partial \phi}{\partial w_{\alpha}}\right)\right|_{\partial \Sigma} \cdot \mathbf{n}_{\Sigma}|d z|^{2}
\end{aligned}
$$

where $\mathbf{n}_{\Sigma}$ represents the normal direction on the boundary of $\Sigma$. On $\partial \Sigma$, we have

$$
\begin{aligned}
g_{\phi, \alpha \bar{\beta}}(z, \cdot) & =g_{0_{\alpha} \bar{\beta}}(z, \cdot)+\sqrt{-1} \partial \bar{\partial} \phi(z, \cdot) \\
& =g_{0_{\alpha} \bar{\beta}}(z, \cdot)+\sqrt{-1} \partial \bar{\partial} \phi_{0}(z, \cdot) \geq \delta g_{0_{\alpha} \bar{\beta}} .
\end{aligned}
$$

Thus,

$$
\begin{aligned}
\int_{\Sigma} 1 & =|\Sigma|+\left.\int_{\partial \Sigma}\left(\frac{\partial \phi}{\partial z}-g_{\phi}^{\alpha \bar{\beta}} \frac{\partial^{2} \phi}{\partial z \partial \beta} \frac{\partial \phi}{\partial w_{\alpha}}\right)\left|\partial \Sigma \cdot \mathbf{n}_{\Sigma}\right| d z\right|^{2} \\
& \leq L_{1}(\delta, \Lambda) .
\end{aligned}
$$

Now we return to the proof of Theorem 5.0.16. Note that

$$
F^{*} \omega_{\phi}^{n}=\omega_{\phi_{0}\left(z_{0}\right)}^{n}=\omega_{0}^{n},
$$

and

$$
\begin{aligned}
F^{*} \omega_{\phi_{0}\left(z_{0}\right)}^{n} & =F^{*}\left(\frac{\omega_{0}^{n}}{\omega_{\phi}^{n}} \omega_{\phi}^{n}\right) \\
& =\left(\frac{\omega_{0}^{n}}{\omega_{\phi}^{n}}\right) \circ F \cdot \omega_{0}^{n} .
\end{aligned}
$$


Thus

$$
\begin{aligned}
\int_{M} \int_{\Sigma}\left(\frac{\omega_{0}^{n}}{\omega_{\phi}^{n}}\right) \circ F \frac{\sqrt{-1}}{2} d z \wedge d \bar{z} z \omega_{0}^{n} & =\int_{M} \int_{\Sigma}\left(\frac{\omega_{0}^{n}}{\omega_{\phi}^{n}(m)}\right) \circ F \omega_{0}^{n} \frac{\sqrt{-1}}{2} d z \wedge d \bar{z} \\
& =\int_{M} \int_{\Sigma} F^{*} \omega_{0}^{n} \frac{\sqrt{-1}}{2} d z \wedge d \bar{z} \\
& =\int_{\Sigma} \int_{M} \omega_{0}^{n} \frac{\sqrt{-1}}{2} d z \wedge d \bar{z}=C .
\end{aligned}
$$

In other words, we have

$$
\int_{M} \operatorname{Cap}\left(\Sigma_{x}\right) d x=\int_{M} \int_{\Sigma}\left(\frac{\omega_{0}^{n}}{\omega_{\phi}^{n}}\right) \circ F \frac{\sqrt{-1}}{2} d z d \wedge \bar{z} d x \leq C .
$$

where $C$ is a topological constant. This concludes our proof of Theorem 5.0 .16

\subsection{No vertical bubble-Proof of Theorem [5.0.14}

We want to re-phrase Theorem 5.0.14.

Theorem 5.2.1. For any sequence of super regular disks (in a sequence of almost super regular foliations $\left.\left\{\left(\phi_{0}{ }^{(m)}, \mathcal{U}_{\phi_{0}}{ }^{(m)}\right), m \in \mathbb{N}\right\}\right)$, there is no vertical bubble in the limit provided that the corresponding sequence of boundary maps $\phi_{0}{ }^{(m)}$ converges in the $C^{2, \alpha}(\partial \Sigma \times M)$ norm to some potential function $\phi_{0}^{(\infty)}$ in the completion of $\mathcal{H}$ by $C^{2, \alpha}(\partial \Sigma \times M)$ norm.

Proof. Suppose

$$
\begin{aligned}
f^{(m)}: \Sigma & \mapsto \Sigma \times \mathcal{W}_{M} \\
z & \mapsto\left(z, f^{(m)}(z), \zeta^{(m)}\left(f^{(m)}(z)\right)\right), \quad m=1,2, \cdots \infty
\end{aligned}
$$

is a sequence of super regular disks in $\left\{\left(\phi_{0}{ }^{(m)}, \mathcal{U}_{\phi_{0}}{ }^{(m)}\right), m \in \mathbb{N}\right\}$. According to Theorem 5.0.16, there exists two constants $L_{0}, L_{1}$ such that all $\left\{f^{(m)}, m \in \mathbb{N}\right\}$ lies in $\mathcal{C}\left(\delta, \Lambda, L_{0}, L_{1}\right.$. Recalled that

$$
\frac{\partial f^{(m) \alpha}}{\partial z}=-g^{(m) \alpha \bar{\beta}} \frac{\partial^{2} \phi^{(m)}}{\partial z \overline{\partial w_{\beta}}} \text {. }
$$

Here $\zeta$ is the corresponding fibre component of $f^{(m)}(\Sigma)$ in $\mathcal{W}_{M}$. In a local coordinate, we write

$$
\zeta^{(m) i}(z, x)=\frac{\partial\left(\phi^{(m)}+\rho\right)}{\partial w_{i}}(z, x), \quad \forall i=1,2, \cdots n
$$

where $\rho$ is a local Kähler potential for the given form $\omega$. Note that in a uniform size neighborhood of $\pi \circ f^{(m)}(\Sigma) \subset \Sigma \times M$, we have

$$
\left|\phi^{(m)}\right|_{C^{1,1}} \leq L_{0}
$$


In particular, there exists a uniform constant $C(\delta, \Lambda)$ such that

$$
\max _{\partial \Sigma}\left|\frac{\partial f^{(m)}}{\partial z}\right|_{g_{0}} \leq C
$$

Consequently, all bubble points ${ }^{19}$ that occur must occur in the interior of $\Sigma \times M$ (although the bubble may travel to some boundary point in the limit.). We want to show that no such bubble point exists; which in turns implies the present theorem.

Suppose bubbling does occur and there is a sequence of points $\left(z_{m}, x_{m}\right)$ such that

$$
\epsilon_{m}^{-1}=\max _{\Sigma \times M}\left|\frac{\partial f^{(m)}}{\partial z}\right|_{g_{0}}=\left|\frac{\partial f^{(m)}}{\partial z}\left(z_{m}, x_{m}\right)\right|_{g_{0}} \rightarrow \infty .
$$

W.l.o.g, we may assume that $\lim _{m \rightarrow \infty} z_{m}=z_{\infty} \in \Sigma$ and $\lim _{m \rightarrow \infty} x_{m}=x_{\infty} \in M$. We want to argue that there exists a point $z_{m}^{\prime} \in B_{2 \sqrt{\epsilon_{m}}}\left(z_{m}\right) \bigcap \Sigma$ such that the area of $B_{\epsilon_{m}}\left(z_{m}^{\prime}\right) \geq c_{0}$ for some uniform constant $c_{0}>0$. If the area functional were the area of a holomorphic disk in $\Sigma \times \mathcal{W}_{M}$, then this follows from standard literatures in this direction. In our setting, this is still true. This in turn implies that there are at most finite number of bubbles. We give a brief explanation here and leave interested readers to fill in the details.

Set $d_{m}=d\left(z_{m}, \partial \Sigma\right)$. If $d_{m}>\frac{1}{2} \epsilon_{m}$, then an easy calculation implies

$$
A\left(f^{(m)}, B_{\epsilon_{m}}\left(z_{m}\right) \bigcap \Sigma\right) \geq c_{0} .
$$

However, we need to establish inequality (5.7) even when $\frac{d_{m}}{\epsilon_{m}} \rightarrow 0$. In such a case, there must exists another point $z_{m}^{\prime} \in \partial B_{1-d_{m}-\epsilon_{m}}(O) \bigcap B_{\sqrt{\epsilon_{m}}}\left(z_{m}\right)$ such that

$$
\left|\frac{\partial f^{(m)}}{\partial z}\left(z_{m}^{\prime}, x_{m}^{\prime}\right)\right|_{g_{0}}>\frac{\epsilon_{m}}{2}, \quad x_{m}^{\prime}=f^{(m)}\left(z_{m}^{\prime}\right) .
$$

This can be proved by using inequality (5.6) and the maximum principle for holomorphic function along a long strip. The main point is that, for this point $z_{m}^{\prime}$, the inequality (5.7) holds. Consequently, there exists at most a finite number of bubble points. Next we want to argue that there is no bubble point at all. For this purpose, we consider two cases: $z_{\infty} \in \partial \Sigma$ or $z_{\infty} \in \Sigma^{0}$. In both cases, we want to show that the existence of a non trivial bubble must lead to contradiction.

Part I: No bubble in the boundary.

\footnotetext{
${ }^{19} \mathrm{~A}$ point $\left\{\left(z_{m}, x_{m}\right), m \in \mathbb{N}\right\}$ is called bubble point if a) $\left|\frac{\partial f^{(m)}}{\partial z}\left(z_{m}, x_{m}\right)\right|_{g_{0}} \rightarrow \infty$ and b) it is a global maximal of $\left|\frac{\partial f^{(m)}}{\partial z}\right|_{g_{0}}$ in $\Sigma \times M$.
} 
We choose a number $\varsigma \in B_{1}$, to be fixed throughout the following argument. Set

$$
\phi^{(m)}(\zeta)=z_{m}+\zeta \cdot \epsilon_{m}, \quad \forall \zeta \in \mathbf{C}
$$

and

$$
l^{(m)}=f^{(m)} \circ \phi^{(m)}, \quad \text { and } \quad \tilde{z}_{m}=\phi^{(m)}(\varsigma) .
$$

By definition, we have

$$
\left|\tilde{z}_{m}-z_{m}\right| \leq \epsilon_{m} \rightarrow 0, \quad \forall|\varsigma| \leq 1
$$

Thus $\lim _{m \rightarrow \infty} \tilde{z}_{m}=z_{\infty}$. First set

$$
\tilde{x}_{m}=\pi \circ f^{(m)}\left(\phi^{(m)}(\varsigma)\right)
$$

Note that

$$
\begin{aligned}
l^{(m)}(\varsigma) & =\left(\tilde{z}_{m}, \tilde{x}_{m}, \zeta^{(m)}\left(\tilde{z}_{m}, \tilde{x}_{m}\right)\right) \\
& =\left(\phi^{(m)}(\varsigma), \pi \circ f^{(m)}\left(\phi^{(m)}(\varsigma)\right), \zeta^{(m)}\left(\phi^{(m)}(\varsigma), \pi \circ f^{(m)}\left(\phi^{(m)}(\varsigma)\right)\right)\right) .
\end{aligned}
$$

Since $\lim _{m \rightarrow \infty} l^{(m)}=l^{\infty}$, we may assume

$$
\lim _{m \rightarrow \infty} \tilde{x}_{m}=\pi \circ l^{\infty}(\varsigma)
$$

Set

$$
\lim _{m \rightarrow \infty} \zeta^{(m)}\left(\tilde{z}_{m}, \tilde{x}_{m}\right)=\zeta^{\infty}(\varsigma) .
$$

for some function $\zeta^{\infty}$. We want to show

$$
\zeta^{\infty}(\varsigma)=\bar{\partial} \phi^{\infty}\left(z_{\infty}, \pi \circ l^{\infty}(\varsigma)\right) .
$$

Now for any $0<\alpha<1$, there exists a uniform constant $C$ such that

$$
\frac{\left|\zeta^{(m)}\left(\tilde{z}_{m}, \tilde{x}_{m}\right)-\zeta^{(m)}\left(z_{\infty}, \pi \circ l^{\infty}(\varsigma)\right)\right|}{\left(\left|\tilde{z}_{m}-z_{\infty}\right|+\left|\tilde{x}_{m}-\pi \circ l^{\infty}(\varsigma)\right|\right)^{\alpha}}<C .
$$

Since $\lim _{m \rightarrow \infty}\left(\left|\tilde{z}_{m}-z_{\infty}\right|+\left|\tilde{x}_{m}-\pi \circ l^{\infty}(\varsigma)\right|\right)=0$, we have

$$
\lim _{m \rightarrow \infty}\left(\zeta^{(m)}\left(\tilde{z}_{m}, \tilde{x}_{m}\right)-\zeta^{(m)}\left(z_{\infty}, \pi \circ l^{\infty}(\varsigma)\right)\right)=0 .
$$

On the other hand, we have

$$
\begin{aligned}
\lim _{m \rightarrow \infty}\left(\zeta^{(m)}\left(z_{\infty}, \pi \circ l^{\infty}(\varsigma)\right)-\bar{\partial} \phi^{\infty}\left(z_{\infty}, \pi \circ l^{\infty}(\varsigma)\right)\right) \\
\quad=\lim _{m \rightarrow \infty}\left(\bar{\partial} \phi^{(m)}\left(z_{\infty}, \pi \circ l^{\infty}(\varsigma)\right)-\bar{\partial} \phi^{\infty}\left(z_{\infty}, \pi \circ l^{\infty}(\varsigma)\right)\right) \\
\quad=0 .
\end{aligned}
$$


Thus,

$$
\lim _{m \rightarrow \infty}\left(\zeta^{(m)}\left(\tilde{z}_{m}, \tilde{x}_{m}\right)-\bar{\partial} \phi^{\infty}\left(z_{\infty}, \pi \circ l^{\infty}(\varsigma)\right)\right)=0 .
$$

Consequently,

$$
\zeta^{\infty}(\varsigma)=\bar{\partial} \phi^{\infty}\left(z_{\infty}, \pi \circ l^{\infty}(\varsigma)\right)
$$

Thus

$$
l^{\infty}(\varsigma)=\left(z_{\infty}, \pi \circ l^{\infty}(\varsigma), \bar{\partial} \phi^{\infty}\left(z_{\infty}, \pi \circ l^{\infty}(\varsigma)\right)\right) \in\left\{z_{\infty}\right\} \times \Lambda_{z_{\infty}, \phi^{\infty}}\left(z_{\infty}\right) .
$$

Since $\varsigma \in B_{1}$ is chosen randomly, we have

$$
l^{\infty}\left(B_{1}\right) \subset\left\{z_{\infty}\right\} \times \Lambda_{z_{\infty}, \phi^{\infty}\left(z_{\infty}\right)} \subset\left\{z_{\infty}\right\} \times \mathcal{W}_{M} .
$$

Next note that $z_{\infty} \in \partial \Sigma$, we have

$$
\phi^{\infty}\left(z_{\infty}, \cdot\right)=\phi_{0}^{\infty}\left(z_{\infty}, \cdot\right)
$$

Thus

$$
\Lambda_{z_{\infty}, \phi^{\infty}\left(z_{\infty}\right)}=\Lambda_{z_{\infty}, \phi_{0}{ }^{\infty}\left(z_{\infty}\right)} \subset\left\{z_{\infty}\right\} \times \mathcal{W}_{M}
$$

is a totally real sub-manifold. This contradicts with the fact that $l^{\infty}\left(B_{1}\right) \subset\left\{z_{\infty}\right\} \times \mathcal{W}_{M}$ is a holomorphic disk! Consequently, there is no bubble sphere/disk developing at the boundary of $\Sigma$.

Now we proceed to part 2.

Part 2: Non existence of interior bubbles. Suppose that $z_{\infty} \in \Sigma^{0}=\Sigma \backslash \partial \Sigma$. Set

$$
\phi^{(m)}(\varsigma)=z_{m}+\frac{\epsilon_{m}}{\delta_{m}} \varsigma
$$

and $l^{(m)}=f^{(m)} \circ \phi^{(m)}$ for $\epsilon_{m} \ll \delta_{m} \rightarrow 0$. For any $k$ fixed, the following map has a non-trivial limit:

$$
l^{\infty}=\lim _{m \rightarrow \infty} l^{(m)}: B_{k} \subset \bigcup_{m=1}^{\infty} B_{\delta_{m}} \rightarrow \Sigma \times \mathcal{W}_{M} .
$$

Let $k \rightarrow \infty$. Then $l^{\infty}$ defines a holomorphic map from $R^{2}$ to $\mathcal{W}_{M}$ with bounded area in $\Sigma \times M$. Therefore, the image must be a holomorphic $S^{2}$.

Here

$$
\frac{\partial l^{(m) \alpha}}{\partial \varsigma}=\frac{\partial f^{(m) \alpha}}{\partial z} \frac{\epsilon_{m}}{\delta_{m}}=-g^{(m) \alpha \bar{\beta}} \frac{\partial^{2} \phi^{(m)}}{\partial z \overline{\partial w_{\beta}}} \frac{\epsilon_{m}}{\delta_{m}} .
$$

Set

$$
\left\{\begin{array}{l}
\eta^{(m) \alpha}=-g^{(m) \alpha \bar{\beta}} \frac{\partial^{2} \phi^{(m)}}{\partial z \overline{\partial w_{\beta}}} \\
\tilde{\eta}^{(m) \alpha}=-g^{(m) \alpha \bar{\beta}} \frac{\partial^{2} \phi^{(m)}}{\partial z \overline{\partial w_{\beta}}} \frac{\epsilon_{m}}{\delta_{m}} .
\end{array}\right.
$$


By assumption, $\tilde{\eta}^{(m)}$ has a non-trivial limit $\eta^{\infty}$ such that

$$
\frac{\partial l^{(m) \alpha}}{\partial \varsigma}=\eta^{(m) \alpha}, \quad \text { and } \quad \frac{\partial l^{\infty \alpha}}{\partial \varsigma}=\eta^{\infty \alpha}
$$

The above equations imply

$$
\begin{aligned}
\partial_{\varsigma}\left(\frac{\partial \phi^{(m)}}{\partial w_{\bar{\beta}}} \circ l^{(m)}\right) & =\left(\frac{\partial}{\partial \varsigma}+\tilde{\eta}^{(m) \alpha} \frac{\partial}{\partial w_{\alpha}}\right) \frac{\partial \phi^{(m)}}{\partial w_{\bar{\beta}}} \\
& =\frac{\epsilon_{n}}{\delta_{n}} \frac{\partial^{2} \phi^{(m)}}{\partial z \partial w_{\bar{\beta}}}+\tilde{\eta}^{(m) \alpha} \frac{\partial^{2} \phi^{(m)}}{\partial w_{\alpha} \partial w_{\bar{\beta}}} \\
& =\frac{\epsilon_{n}}{\delta_{n}} \frac{\partial^{2} \phi^{(m)}}{\partial z \partial w_{\bar{\beta}}}+\tilde{\eta}^{(m) \alpha}\left(g_{\alpha \bar{\beta}}^{(m)}-g_{0, \alpha \bar{\beta}}\right) \\
& =\frac{\epsilon_{n}}{\delta_{n}} \frac{\partial^{2} \phi^{(m)}}{\partial z \partial w_{\bar{\beta}}}+\left(-g^{(m) \alpha \bar{r}} \frac{\partial^{2} \phi^{(m)}}{\partial z \bar{\partial} w_{r}} \frac{\epsilon_{m}}{\delta_{m}}\right) g_{\alpha \bar{\beta}}^{(m)}-\tilde{\eta}^{(m) \alpha} g_{0, \alpha \bar{\beta}} \\
& =-g_{0, \alpha \bar{\beta}} \tilde{\eta}^{(m) \alpha} .
\end{aligned}
$$

Thus

$$
\begin{aligned}
-g_{0, \alpha \bar{\beta}} \tilde{\eta}^{(m) \alpha} \tilde{\eta}^{(m) \bar{\beta}} & =\partial_{\varsigma}\left(\frac{\partial \phi^{(m)}}{\partial w_{\bar{\beta}}} \circ l^{(m)}\right) \tilde{\eta}^{(m) \bar{\beta}} \\
& =\partial_{\varsigma}\left(\tilde{\eta}^{(m) \bar{\beta}} \frac{\partial \phi^{(m)}}{\partial w_{\bar{\beta}}} \circ l^{(m)}\right) \\
& =\partial_{\varsigma}\left(\partial_{\bar{\varsigma}}\left(\phi^{(m)} \circ l^{(m)}\right)-\frac{\epsilon_{n}}{\delta_{n}} \frac{\partial \phi^{(m)}}{\partial \bar{z}} \circ l^{(m)}\right) \\
& =\partial_{\varsigma} \partial_{\bar{\varsigma}}\left(\phi^{(m)} \circ l^{(m)}\right)-\frac{\epsilon_{n}}{\delta_{n}} \partial_{\varsigma}\left(\frac{\partial \phi^{(m)}}{\partial \bar{z}} \circ l^{(m)}\right) .
\end{aligned}
$$

Let $\chi(\varsigma)$ be any smooth test function which vanish outside a compact domain of $R^{2}$. For $m$ large enough, the domain of $\chi$ is contained inside in the domain of $l^{(m)}$. Then,

$$
\begin{aligned}
-\int_{R^{2}} \chi & g_{0, \alpha \bar{\beta}} \tilde{\eta}^{(m) \alpha} \tilde{\eta}^{(m) \bar{\beta}}|d \varsigma|^{2} \\
& =\int_{R^{2}} \chi \partial_{\varsigma} \partial_{\bar{\varsigma}}\left(\phi^{(m)} \circ l^{(m)}\right)|d \varsigma|^{2}-\int_{R^{2}} \chi \frac{\epsilon_{m}}{\delta_{m}} \partial_{\varsigma}\left(\frac{\partial \phi^{(m)}}{\partial \bar{z}} \circ l^{(m)}\right)|d \varsigma|^{2} \\
& =-\int_{R^{2}} \frac{\partial \chi}{\partial \varsigma} \partial_{\bar{\varsigma}}\left(\phi^{(m)} \circ l^{(m)}\right)|d \varsigma|^{2}+\frac{\epsilon_{m}}{\delta_{m}} \int_{R^{2}} \frac{\partial \chi}{\partial \varsigma} \frac{\partial \phi^{(m)}}{\partial \bar{z}} \circ l^{(m)}|d \varsigma|^{2}
\end{aligned}
$$

Taking limit as $m \rightarrow \infty$, we have

$$
-\int_{R^{2}} \chi g_{0, \alpha \bar{\beta}} \tilde{\eta}^{\infty \alpha} \tilde{\eta}^{\infty \bar{\beta}}|d \varsigma|^{2}=-\int_{R^{2}} \frac{\partial \chi}{\partial \varsigma} \partial_{\bar{\varsigma}}\left(\phi^{\infty} \circ l^{\infty}\right)|d \varsigma|^{2} .
$$

This holds for any test function in $\mathbb{R}^{2}$. Now the image of $l^{\infty}$ is a smooth $S^{2}$ in $\left\{z_{\infty}\right\} \times M$. Therefore, $l^{\infty *} \omega_{0}$ is an induced (smooth) Kähler form in this class. We may as well assume $l^{\infty *} \omega_{0}$ is cohomologous to the standard Kähler form in $S^{2}$. Then there exists a bounded smooth function $\lambda$ in this $S^{2}$ such that

$$
\begin{aligned}
l^{\infty *} \omega_{0} & =g_{0, \alpha \bar{\beta}} \tilde{\eta}^{\infty \alpha} \tilde{\eta}^{\infty \bar{\beta}}|d \varsigma|^{2} \\
& =\frac{\partial^{2}}{\partial \varsigma \partial \bar{\varsigma}}\left(-2 \ln \left(1+|\varsigma|^{2}\right)+\lambda \circ l^{\infty}\right) d \varsigma d \bar{\varsigma}
\end{aligned}
$$


Then

$$
\int_{R^{2}} \frac{\partial \chi(\varsigma)}{\partial \varsigma} \cdot \frac{\partial}{\partial \varsigma}\left(-2 \ln \left(1+|\varsigma|^{2}\right)+\lambda \circ l^{\infty}+\phi^{\infty} \circ l^{\infty}\right)|d \varsigma|^{2}=0
$$

Set

$$
\Phi=\left(-2 \ln \left(1+|\varsigma|^{2}\right)+\lambda \circ l^{\infty}+\phi^{\infty} \circ l^{\infty}\right) .
$$

Since $\chi$ is an arbitrary test function, this implies that $\Phi$ is a weakly $C^{1,1}$, one side bounded harmonic function in $\mathbf{R}^{2}$. Then $\Phi$ must be a constant function $c$. Then

$$
\phi^{\infty} \circ l^{\infty}=c+2 \ln \left(1+|\varsigma|^{2}\right)+\lambda \circ l^{\infty}
$$

is not a bounded function as $\varsigma \rightarrow \infty$. This contradicts with the fact that $\phi^{\infty}$ is uniformly bounded, which in turn implies that there is no bubble in the interior.

The proof of this theorem is then completed.

\subsection{Proof of Theorem 5.0.15}

\subsubsection{Uniform $C^{1}$ transversal derivatives of almost super regular foliations}

In this subsection, we continue to derive the a priori regularity estimate for the disk with uniform upper bound on area and capacity.

Theorem 5.3.1. Let $\Omega$ be any compact sub-domain in $\Sigma^{0}$. For any holomorphic disk $f \in$ $\mathcal{C}\left(\delta, \Lambda, L_{0}, L_{1}, L_{2}\right)$, there exists a constant $C>1$ such that:

$$
\frac{1}{C} \leq\left(\frac{\omega_{0}^{n}}{\omega_{\phi}^{n}}\right) \circ f \leq C .
$$

Here $C$ depends on $\delta, \Lambda, L_{0}, L_{2}$ and $d(\partial \Omega, \partial \Sigma)$. Moreover, this constant approaches to $\infty$ if $d(\partial \Omega, \partial \Sigma) \rightarrow 0$.

Proof. Proposition 4.2 .13 implies that the trace of curvature of the $T M$ bundle over the disk $\Sigma$ has interior estimates:

$$
0 \leq S \circ f(z, \cdot) \leq \frac{C_{1}}{d(z, \partial \Sigma)^{2}} .
$$

Corollary 4.2.11 then implies

$$
0 \leq \partial_{z} \bar{\partial}_{z}\left(\ln \frac{\omega_{\phi}^{n}}{\omega_{0}^{n}}\right) \leq C_{2}, \quad \forall z \in \Omega,
$$


for some constants $C_{2}$ which depends on $C_{1}$. On the other hand, finite capacity implies

$$
\int_{\Omega}\left(\frac{\omega_{0}^{n}}{\omega_{\phi}^{n}}\right) \circ f \frac{\sqrt{-1}}{2} d z d \bar{z} \leq L_{2} .
$$

This in turn implies that in a slightly smaller sub-domain $\Omega_{1} \subsetneq \Omega$, we have

$$
\left|\ln \frac{\omega_{\phi}^{n}}{\omega_{0}^{n}}-C_{3}\right| \leq C_{4}
$$

for some constant $C_{3}$ which might depends on $\phi$. The Key observation is that $C_{4}$ depends only on $C_{2}, C_{3}$ and $L_{2}$. Consequently, there exists a constant $C_{5}$ such that

$$
\frac{1}{C_{5}} \leq\left(\frac{\omega_{0}^{n}}{\omega_{\phi}^{n}}\right) \circ f \leq C_{5} .
$$

Here $C_{5}$ depends only on $C_{1}, L_{2}$.

Note that $\nabla_{\partial}, \nabla_{\bar{\partial}}$ induces naturally a map from $T M$ to $T M \otimes T^{*} M$. The image of the leaf vector field $X$ under these two operators are of particular interest. In a local coordinate chart, we have

$$
\nabla_{\partial} X=\left(\frac{\partial \eta^{\alpha}}{\partial w^{\beta}}+\eta^{\nu} \Gamma_{\nu \beta}^{\alpha}\left(g_{0}\right)\right) \cdot \frac{\partial}{\partial w^{\alpha}} \bigotimes d w^{\beta}=\eta_{, \beta}^{\alpha} \frac{\partial}{\partial w^{\alpha}} \bigotimes d w^{\beta}
$$

and

$$
\nabla_{\bar{\partial}} X=\frac{\partial \eta^{\alpha}}{\partial w^{\bar{\beta}}} \cdot \frac{\partial}{\partial w^{\alpha}} \bigotimes d w^{\bar{\beta}}=\eta_{, \bar{\beta}}^{\alpha} \frac{\partial}{\partial w^{\alpha}} \bigotimes d w^{\bar{\beta}}
$$

Theorem 5.3.2. For any super regular disk $f \in \mathcal{C}\left(\delta, \Lambda, L_{0}, L_{1}, L_{2}\right)$, the $T^{*(1,0)} M$ component of the first transversal derivatives of the leaf vector field is bounded in any compact sub-domain $\Omega \subset \Sigma$. Namely, there exists a constant $C$ depends on $\delta, \Lambda, L_{0}, L_{1}, L_{2}$ and $d(\partial \Omega, \partial \Sigma)$, such that (c.f. equation (5.5))

$$
\left\|\nabla_{\partial} X\right\|_{g_{0}}<C, \quad \text { in } \Omega .
$$

Moreover, this constant $C$ blows up if $d(\partial \Omega, \partial \Sigma) \rightarrow 0$.

Proof. For any $z \in \Omega$, consider function $d(z, \partial \Omega) \cdot\left\|\nabla_{\partial} X\right\|_{g_{0}}(z)$. This is a non-negative function in $\Omega$ which vanishes on $\partial \Omega$. The maximum value must be attained in $\Omega^{0}$. If this theorem is false, then there exists a sequence of super regular holomorphic disks $\left\{f^{(m)}\right\} \subset \mathcal{C}\left(\delta, \Lambda, L_{0}, L_{1}, L_{2}\right)$ such that

$$
\lim _{m \rightarrow \infty} \max _{\Omega} d(z, \partial \Omega) \cdot\left\|\nabla_{\partial} X^{(m)}\right\|_{g_{0}}(z)=\infty .
$$

Without loss of generality, one may assume that the maximum is attained at the point $z_{m}$. Set

$$
\lim _{m \rightarrow \infty} z_{m}=z_{\infty} \in \bar{\Omega}
$$


On the other hand, Theorem 5.0 .14 implies that there exists a subsequence of $f^{(m)}$ which converges in $\mathcal{C}\left(\delta, \Lambda, L_{0}, L_{1}, L_{2}\right)$ as an embedded holomorphic disk. W.l.o.g., we may assume that $f^{(m)}$ is fixed but the restricted TM bundle varies. Denote the sequence of re-scaling factors as

$$
\frac{1}{\epsilon_{m}}=\left\|\nabla_{\partial} X\right\|_{g_{0}}\left(z_{m}\right) \rightarrow \infty
$$

Write this sequence of disks as

$$
\begin{aligned}
f^{(m)}: \Sigma & \mapsto \Sigma \times M & \hookrightarrow \Sigma \times \mathcal{W}_{M} \\
z & \mapsto\left(z, f^{(m)}(z)\right) & \hookrightarrow\left(z, f^{(m)}(z), \xi^{(m)}\right)
\end{aligned}
$$

where

$$
\xi^{(m) \alpha}(z)=\frac{\partial(\rho+\phi)}{\partial w_{\alpha}} \circ f^{(m)}(z), \quad \forall \alpha=1,2, \cdots n
$$

Moreover

$$
X^{(m)}=\sum_{i=1}^{n} \eta^{(m) i} \frac{\partial}{\partial w_{i}}
$$

and

$$
\eta^{(m) \alpha}=\frac{\partial f^{(m) \alpha}}{\partial z}=-g^{(m) \alpha \bar{\beta}} \frac{\partial^{2} \phi^{(m)}}{\partial z \overline{\partial w_{\beta}}} .
$$

Theorem 5.3.1 implies that there is a positive number $C_{3}>0$ such that

$$
\frac{1}{C} \leq\left(\frac{\omega_{0}^{n}}{\omega_{\phi^{(m)}}^{n}}\right) \circ f^{(m)} \leq C_{3}
$$

hold uniformly in $\Omega$ since $d(\Omega, \partial \Sigma)>0$. Combining this with the $C^{1,1}$ estimate in [8], there exists a small positive constant $\epsilon_{0}>0$, such that

$$
\epsilon_{0}\left(g_{0, i \bar{j}}\right)_{n \times n} \leq\left(g_{i \bar{j}}^{(m)}\right)_{n \times n} \leq C\left(g_{0, i \bar{j}}\right)_{n \times n}
$$

holds for these disks on $\Omega$. Set

$$
\tilde{\phi}^{(m)}(z, w)=\phi^{(m)}\left(z_{m}+\epsilon_{m} \cdot z, w\right), \quad \tilde{X}^{(m)}=\sum_{\alpha=1}^{n} \tilde{\eta}^{(m) \alpha} \frac{\partial}{\partial w_{\alpha}}
$$

where

$$
\begin{aligned}
\tilde{\eta}^{(m) \alpha}(z, w) & =g^{(m) \alpha \bar{\beta}} \frac{\partial^{2} \tilde{\phi}^{(m)}}{\partial z \partial w_{\bar{\beta}}} \\
& =\epsilon_{m} \eta^{(m) \alpha}\left(\epsilon_{m} \cdot z+z_{m}, w\right) .
\end{aligned}
$$

Moreover,

$$
\left\|\nabla_{\partial} \tilde{X}^{(m)}\right\|_{g_{0}}(0)=\epsilon_{m} \cdot\left\|\nabla_{\partial} X^{(m)}\right\|_{g_{0}}\left(z_{m}\right)=1 .
$$


Set

$$
\Omega^{(m)}=\left\{z \mid \epsilon_{m} \cdot z+z_{m} \in \Omega\right\}
$$

Clearly

$$
\begin{aligned}
\lim _{m \rightarrow \infty} d\left(0, \partial \Omega^{(m)}\right) & =\lim _{m \rightarrow \infty} d\left(0, \partial \Omega^{(m)}\right) \cdot\left|\nabla_{\partial} \tilde{X}^{(m)}\right|_{g_{0}}(0) \\
& =\lim _{m \rightarrow \infty} d\left(z_{m}, \partial \Omega\right) \cdot\left\|\nabla_{\partial} X^{(m)}\right\|_{g_{0}}\left(z_{m}\right)=\infty .
\end{aligned}
$$

In other words,

$$
\Omega^{(m)} \rightarrow \mathbb{R}^{2}
$$

Here

$$
\begin{aligned}
\tilde{S}^{(m)}(z, w) & =\frac{\partial \tilde{\eta}^{(m) \bar{\beta}}}{\partial w_{\alpha}} \frac{\partial \tilde{\eta}^{(m) \alpha}}{\partial \bar{w}_{\beta}} \\
& =\epsilon_{m}^{2} \frac{\partial \eta^{(m) \beta}}{\partial w_{\alpha}} \frac{\partial \eta^{(m) \alpha}}{\partial w_{\beta}} \\
& =\epsilon_{m}^{2} \cdot S^{(m)}\left(z_{m}+\epsilon_{m} z, w\right) .
\end{aligned}
$$

Then $\tilde{S}^{(m)}$ still satisfies the inequality

$$
\partial_{z} \partial_{\bar{z}} \tilde{S}^{(m)} \geq \frac{2}{n} \tilde{S}^{(m) 2}
$$

in $\Omega^{(m)}$. Consequently, we have (cf. Proposition. 4.2.13)

$$
0 \leq \tilde{S}^{(m)}(z) \leq \frac{C}{d\left(z, \partial \Omega^{(m)}\right)^{2}} \rightarrow 0, \quad \forall z \in \Omega^{(k)}
$$

for any fixed $k$ and $m \rightarrow \infty$. Recall that

$$
\begin{aligned}
\tilde{S}^{(m)} & =\epsilon_{m}^{2} \frac{\partial \eta^{(m) \alpha}}{\partial w_{\bar{\beta}}} \frac{\partial \eta^{(m) \bar{\beta}}}{\partial w_{\alpha}} \\
& =\epsilon_{m}^{2} g^{(m) \alpha \bar{a}} g^{(m) \bar{\beta} b}\left(\frac{\partial \phi^{(m)}}{\partial z}\right)_{, \bar{a} \bar{\beta}}\left(\frac{\partial \phi^{(m)}}{\partial \bar{z}}\right)_{, \alpha a} \\
& \geq C^{-2} \epsilon_{m}^{2} g_{0}^{\alpha \bar{a}} g_{0}^{\bar{\beta} b}\left(\frac{\partial \phi^{(m)}}{\partial z}\right)_{, \bar{a} \bar{\beta}}\left(\frac{\partial \phi^{(m)}}{\partial \bar{z}}\right)_{, \alpha a} .
\end{aligned}
$$

The last inequality holds because $g_{\alpha \bar{\beta}}^{(m)}$ has a uniform upper bound. Thus,

$$
\lim _{m \rightarrow \infty} \epsilon_{m} \cdot\left(\frac{\partial \phi^{(m)}}{\partial \bar{z}}\right)_{, \alpha a}=0 .
$$

Consequently, we have

$$
\begin{aligned}
\lim _{m \rightarrow \infty} \frac{\partial \tilde{\eta}^{(m) \alpha}}{\partial w_{\bar{\beta}}} & =\lim _{m \rightarrow \infty} \epsilon_{m} \cdot \frac{\partial \eta^{(m) \alpha}}{\partial w_{\bar{\beta}}} \\
& =\lim _{m \rightarrow \infty} \epsilon_{m} \cdot g^{(m) \alpha \bar{a}}\left(\frac{\partial \phi^{(m)}}{\partial z}\right)_{, \bar{\beta} \bar{a}}=0 .
\end{aligned}
$$


The last inequality holds since $g_{\alpha \bar{\beta}}^{(m)}$ has a uniform positive lower bound on $\Omega$.

Moreover, for any fixed $z$, we have

$$
\left\|\nabla_{\partial} \tilde{X}^{(m)}\right\|_{g_{0}}(z) d\left(z, \partial \Omega^{(m)}\right) \leq\left\|\nabla_{\partial} X^{(m)}\right\|_{g_{0}}(0) d\left(0, \partial \Omega^{(m)}\right) .
$$

Therefore ${ }^{20}$

$$
\begin{aligned}
\left\|\nabla_{\partial} \tilde{X}^{(m)}\right\|_{g_{0}}(z) & \leq\left\|\nabla_{\partial} \tilde{X}^{(m)}\right\|_{g_{0}}(0) \frac{d\left(0, \partial \Omega^{(m)}\right)}{d\left(z \partial \Omega^{(m)}\right)} \\
& \leq 2 \cdot\left\|\nabla_{\partial} \tilde{X}^{(m)}\right\|_{g_{0}}(0) \\
& \leq 4
\end{aligned}
$$

Consequently, $\nabla_{\partial} \tilde{X}^{(m) 21}$ is uniformly bounded and $\left|\nabla_{\partial} \tilde{X}^{(m)}\right|_{g_{0}} \approx 1$ at the origin. By Lemma 4.2.7 both $\frac{\partial \tilde{\eta}^{(m)}}{\partial w_{i}}$ and $\frac{\partial \tilde{\eta}^{(m)}}{\partial w_{i}}$ are uniformly $C^{\alpha}(\forall \alpha<1)$ bounded. Since $g_{i \bar{j}}^{(m)}$ has a uniform upper and lower bound in $\Omega$, we have

$$
\lim _{m \rightarrow \infty} \frac{\partial \tilde{\eta}^{(m)}}{\bar{\partial} w_{i}}(z, \cdot)=0
$$

On the other hand, $\frac{\partial \tilde{\eta}^{(m)}}{\partial w_{i}}$ is a bounded holomorphic function in the limit since $\frac{\partial \tilde{\eta}^{(m)}}{\partial \bar{w}_{i}}=0$ in the limit (cf. Lemma 4.2.7). Therefore, $\frac{\partial \tilde{\eta}^{(m)}}{\partial w_{i}}$ is a constant matrix everywhere in the limit! Set

$$
g_{i \bar{j}}^{\infty}=\lim _{m \rightarrow \infty} g_{i \bar{j}}^{(m)}, \quad \tilde{\eta}^{\infty}=\lim _{m \rightarrow \infty} \tilde{\eta}^{(m)} .
$$

Then

$$
\frac{1}{C} I_{n \times n} \leq\left(g_{i \bar{j}}^{(m)}\right) \leq C I_{n \times n}
$$

Theorem 4.2.8 takes the form

$$
\partial_{z} \overline{\partial_{z}} g_{, i j}^{\infty}=a_{i}^{\alpha} \overline{a^{\beta}}{ }_{j} g_{, \bar{\beta} \alpha}^{\infty}
$$

where

$$
\frac{\partial \tilde{\eta}^{\infty \alpha}}{\partial w_{i}}=a^{\alpha}{ }_{i}, \quad \text { and } \quad \frac{\partial \tilde{\eta}^{\infty \bar{\beta}}}{\overline{\partial w_{j}}}=\overline{a^{\beta}}
$$

\footnotetext{
${ }^{20}$ The second inequality holds since $\lim _{m \rightarrow \infty} d\left(0, \partial \Omega^{(m)}\right)=\infty$ while $d(0, z)=|z|$ is fixed.

${ }^{21}$ Note that

$$
\tilde{\eta}_{, i}^{(m) \alpha}=\frac{\partial \tilde{\eta}^{(m) \alpha}}{\partial w_{i}}+\tilde{\eta}^{(m) \beta} \Gamma_{\beta i}^{\alpha}\left(g_{0}\right) .
$$
}

where

$$
\lim _{m \rightarrow \infty} \tilde{\eta}^{\beta} \Gamma_{\beta i}^{\alpha}\left(g_{0}\right)=\lim _{m \rightarrow \infty} \epsilon_{m} \cdot \eta^{(m) \beta} \Gamma_{\beta i}^{\alpha}\left(g_{0}\right)=0 .
$$

This is because the disk, when restricted in $\Omega$, uniformly converge to a smooth limit surface. Hence $\Gamma_{\beta i}^{\alpha}\left(g_{0}\right)$ is uniformly bounded. Thus,

$$
\frac{\partial \tilde{\eta}^{(m)}}{\partial w_{i}} \approx \tilde{\eta}_{, i}^{(m) \alpha} .
$$


are constant matrixes. This clearly violates the maximum principle. Note that $g_{i \bar{j}}^{\infty}$ and its derivatives are uniformly bounded in the entire plan. The contradiction implies the constant matrix $\left(a_{i}^{\alpha}\right)$ must vanish identically which contradicts the blowingup assumption.

Following Prop. 4.2.13, we can easily derive the following

Corollary 5.3.3. For any super regular disk $f \in \mathcal{C}\left(\delta, \Lambda, L_{0}, L_{1}, L_{2}\right)$, the $T^{*}(0,1) M$ component of the first transversal derivatives of the leaf vector field is bounded in any compact sub-domain $\Omega \subset \Sigma$. Namely, there exists a constant $C$ depending only on $\delta, \Lambda, L_{0}, L_{1}, L_{2}$ and $d(\partial \Omega, \partial \Sigma)$, such that (c.f. equation (5.5))

$$
\left\|\nabla_{\bar{\partial}} X\right\|_{g_{0}}<C, \quad \text { in } \Omega .
$$

Moreover, this constant $C$ blows up if $d(\partial \Omega, \partial \Sigma) \rightarrow 0$.

\subsubsection{The Limit of Super-regular disks with finite capacity is Super regular}

Theorem 5.3.4. Suppose that $\left\{\phi_{0}{ }^{(m)}, m \in \mathbb{N}\right\}$ is a sequence of loops in $C^{2, \alpha}(\partial \Sigma, \mathcal{H})$ which satisfies the uniform bound (5.3) and $f^{(m)}:(\Sigma, \partial \Sigma) \rightarrow\left(\Sigma \times \mathcal{W}_{M}, \bar{\Lambda}_{\phi_{0}(m)}\right)$ is a sequence of super-regular holomorphic disks in $\mathcal{C}\left(\delta, \Lambda, L_{0}, L_{1}, L_{2}\right)$. There exists a subsequence of $\left\{f_{m}\right\}$ which converges to an embedded, super-regular holomorphic disk.

Note that this in fact implies Theorem 5.0.15.

Proof. We use $1 \leq i, j, k, \alpha, \beta, \gamma \leq n$ to denote the labels on Kähler manifold $M$, and use $1 \leq p, q, \cdots \leq 2 n$ to denote the labels in the moduli space $\mathcal{U}_{\phi_{0}}$. By definition, we have

$$
\frac{\partial F^{(m) i}}{\partial \bar{z}}=0, \quad \forall i=1,2, \cdots
$$

Let $\nu_{p}^{(m)}=\left\|\frac{\partial F^{(m)}}{\partial t_{p}}\right\|_{W^{1,2}(\Sigma)}$. Following the standard theory on elliptic problems, there exists a uniform constant $C(\delta, \Lambda)$ (which depends on the totally real boundary sub-manifold) such that

$$
\left\|\frac{\partial F^{(m)}}{\partial t_{p}}\right\|_{L^{\infty}}<C \cdot \nu_{p}^{(m)} .
$$

On the other hand, we have

$$
\begin{aligned}
\frac{\partial}{\partial z} \frac{\partial F^{(m) i}}{\partial t_{p}} & =\frac{\partial \eta^{(m) i}}{\partial w_{\alpha}} \frac{\partial F^{(m) \alpha}}{\partial t_{p}}+\frac{\partial \eta^{(m) i}}{\partial w_{\bar{\beta}}} \frac{\partial F^{(m) \bar{\beta}}}{\partial t_{p}} \\
\frac{\partial}{\partial \bar{z}} \frac{\partial F^{(m) i}}{\partial t_{p}} & =0
\end{aligned}
$$


By Theorem 5.3.2 we have

$$
\left|\frac{\partial \eta^{(m) i}}{\partial w_{\alpha}}\right|_{g_{0}}+\left|\frac{\partial \eta^{(m) i}}{\partial w_{\bar{\beta}}}\right|_{g_{0}} \leq C
$$

uniformly in any sub-domain $\Omega \subsetneq \Sigma$ such that $d(\partial \Omega, \partial \Sigma)>0$. Fix $\Omega$ now.

For any $z_{0} \in \Omega$, and we re-parametrize the family of disks such that for

$$
F^{(m)}\left(z_{0}, p\right)=\left(z_{0}, x(p)\right)
$$

for any $\left(z_{0}, t\right)$ in the domain of $F^{(m)}$. This is possible since $f^{(m)}$ is a super regular disk. By definition, we have

$$
\left(\frac{\partial F^{(m) i}}{\partial t_{p}} \frac{\partial F^{(m) \bar{i}}}{\partial t_{p}}\right)_{2 n \times(2 n)}
$$

is non-singular at $z=z_{0}$. From the equation (5.9) and a priori estimate (5.10), we have the following important Harnack type inequality

$$
C^{-1} \cdot\left|\frac{\partial F^{(m)}}{\partial t_{p}}\right|_{g_{0}}\left(z_{1}\right) \leq\left|\frac{\partial F^{(m)}}{\partial t_{p}}\right|_{g_{0}}\left(z_{2}\right) \leq C \cdot\left|\frac{\partial F^{(m)}}{\partial t_{p}}\right|_{g_{0}}\left(z_{1}\right), \quad \forall z_{1}, \quad z_{2} \in \Omega .
$$

Here $C$ depends on $\Omega$ only. Consequently, we have shown that

$$
\left(\frac{\partial F^{(m) i}}{\partial t_{p}} \frac{\partial F^{(m) \bar{i}}}{\partial t_{p}}\right)_{2 n \times(2 n)}
$$

is a bounded and non-singular matrix in $\Omega$ In particular, there exists a small constant $c$ such that

$$
\left|\operatorname{det}\left(\frac{\partial F^{(m) i}}{\partial t_{p}} \frac{\partial F^{(m) \bar{i}}}{\partial t_{p}}\right)_{2 n \times(2 n)}\right|>c .
$$

Here $c$ depend only on (5.9). This implies that $\nu_{p}(1 \leq p \leq 2 n)$ has a uniform positive low bound. Now we claim that they all have uniform upper bound:

$$
\sup _{m \rightarrow \infty} \nu_{p}^{(m)}<\infty
$$

Otherwise, there exists a subsequence (use the same notation for convenience) such that

$$
\lim _{m \rightarrow \infty} \nu_{p}^{(m)}=\infty
$$


The matrix

$$
\left(\frac{\partial F^{(m) i}}{\partial t_{p}} \frac{\partial F^{(m) \bar{i}}}{\partial t_{p}}\right)_{2 n \times(2 n)}
$$

is uniformly bounded from above and below on $\Omega$. Set

$$
u_{p}^{(m)}=\left(u_{p}^{(m) 1}, u_{p}^{(m) 2}, \cdots u_{p}^{(m) n}\right)
$$

where

$$
u_{p}^{(m) i}=\frac{\frac{\partial F^{(m) i}}{\partial t_{p}}}{\nu_{p}^{(m)}} .
$$

Then, $u_{p}^{(m)} \rightarrow 0$ in $\Omega$ uniformly. Since $u_{p}^{(m)}$ is holomorphic on $\Sigma$, then $u_{p}^{(m)}$ converges to 0 at least in $\Sigma^{0}$. This contradicts the fact that $u_{p}^{(m)}$ has a non-zero limit. Consequently, our claim holds and $\nu_{p}$ has a uniform upper bound and

$$
\left(\frac{\partial F^{(m) i}}{\partial t_{p}} \frac{\partial F^{(m) \bar{i}}}{\partial t_{p}}\right)_{2 n \times(2 n)}
$$

is uniformly bound from above on $\Sigma$. Recall that $F^{*} \omega_{\phi^{(m)}}$ is an invariant form along disc direction. In particular, the pulled back of volume form is constant along the disc. Thus, we have

$$
\begin{aligned}
\operatorname{det}\left(g_{p q}^{(m)}\right)\left(z_{0}\right) & =\operatorname{det}\left(g_{i \bar{j}}^{(m)}\right)(z) \operatorname{det}\left(\frac{\partial F^{(m) i}}{\partial t_{p}} \frac{\partial F^{(m) \bar{j}}}{\partial t_{p}}\right)(z) \\
& \leq C \operatorname{det}\left(g_{i \bar{j}}^{(m)}\right)(z), \quad \forall z \in \Sigma .
\end{aligned}
$$

Thus $g_{i \bar{j}}^{(m)}$ has a uniform positive lower bound on $\Sigma$. According to Donaldson [11], the limiting disk must be super-regular.

\subsection{Proof of Theorem 5.0.17; Compactness of almost super regular foliations}

We give a proof directly based on our work in the previous two subsections.

Proof. As before, identify $\mathcal{U}_{\phi_{0}(m)}$ with an open dense set $\tilde{M}$ of $M$. For every point $x$ in $\tilde{M}$, consider $g_{x}$ as the holomorphic disk in $\mathcal{U}_{\phi_{0}}{ }^{(m)}$ which passes through the point $\left(z_{0}, x\right)$. Set

$$
h^{(m)}(x)=\operatorname{Cap}\left(g_{x}^{(m)}(\Sigma)\right) .
$$

Theorem 5.0.16 then implies that

$$
\int_{\tilde{M}} h^{(m)}(x) d x=\int_{\tilde{M}} \operatorname{Cap}\left(g_{x}^{(m)}(\Sigma)\right) d x \leq C .
$$


Therefore, for generic points $x \in \tilde{M}$, there exists a subsequence of $g_{x}^{(m)}$ such that

$$
h^{(m)}(x)=\operatorname{Cap}\left(g_{x}^{(m)}\right) \leq C(x), \quad \forall m=1,2, \cdots \infty .
$$

According to Theorem 5.0.15] after passing to a subsequence if necessary, this sequence $\left\{g_{x}^{(m)}, m \in\right.$ $\mathbb{N}\}$ of disks has a uniform limit $g_{x}^{\infty}$. Moreover, the limit disc is super-regular, in particular, regular. Therefore, there exists a small open subset $B_{r(x)}(x)$ in the moduli space such that the foliation $\left.F^{(m)}\right|_{\Sigma \times B_{r(x)}(x)}: \Sigma \times B_{r(x)}(x) \rightarrow \Sigma \times \mathcal{W}_{M}$ has a unique smooth limit $F^{(\infty)}: \Sigma \times B_{r(x)}(x) \rightarrow$ $\Sigma \times \mathcal{W}_{M}$. Moreover, $\pi \circ F^{(\infty)}$ induces a foliation in a small open, tublar neighborhood of $g_{x}^{(\infty)} \subset \Sigma \times M$. Consequently, $h^{(m)}$ is uniformly continuous in $B_{r(x)}(x) \subset \tilde{M}$. Following Lemma 5.4 .1 below, there exists a set $E$ of measure 0, and a subsequence of $h^{(m)}$ (ultimately of $g_{x}^{(m)}$ ) such that $h^{(m)}$ is uniformly continuous function in any compact subset of $\tilde{M} \backslash E$. Theorem 5.0.15 again implies that the foliation has a uniform limit in this compact subset. We define $\mathcal{U}_{\phi_{0}(\infty)}=\lim _{m \rightarrow \infty} F^{(m)}: \Sigma \times \tilde{M} \backslash E \rightarrow \Sigma \times \mathcal{W}_{M}$

For every $x \in E$, any disk in $\left\{g_{x}^{(m)}, m \in \mathbb{N}\right\}$ has a uniform upper bound on its area. Following Theorem 5.0.14 there exists a subsequence of disks $\left\{g_{x}^{(m)}, m=1,2, \cdots \infty\right\}$ which converges to an embedded holomorphic disk $S_{x}^{(\infty)}$. This limit depends on the selection of subsequence apriori and may not be unique in general. However, the image of each limiting disk $S_{x}^{(\infty)}$ does not intersect the image of any disk of $\mathcal{U}_{\phi_{0}} \infty$ on $\Sigma^{0} \times M$.

Let us expand the open set $F^{(\infty)} \subset \mathcal{M}_{\phi_{0}^{(\infty)}}$ by including $E_{\infty}=\bigcup_{x \in E} S_{x}^{(\infty)}$. Then, $F^{(\infty)}$ is a partially smooth foliation (c.f. defi. 3.4.7), where $\mathcal{U}_{\phi_{0}(\infty)}$ is the set of super regular disks in $F^{(\infty)}$.

The only thing remain is to show that $\omega_{\phi_{0}(\infty)}^{n}$ is a continuous form on $\Sigma^{0} \times M$. As before, denote the image of super regular discs under the evaluation map $\pi \circ$ ev as $\mathcal{V}_{\phi_{0}}$. Let $\mathcal{S}_{\phi_{0}}=$ $\Sigma \times M \backslash \mathcal{V}_{\phi_{0}}$ be the union of all of the singular points. Clearly, $\omega_{\phi_{0}(\infty)}^{n}$ is a smooth $(n, n)$ form in $\mathcal{V}_{\phi_{0}}$ and vanishes completely in its complement $\mathcal{S}_{\phi_{0}}$.. To show that this $(n, n)$ form is continuous, we just need to verify that, for any sequence $\left(z_{i}, x_{i}\right) \in \mathcal{V}_{\phi_{0}}$ which converges to $(\bar{z}, \bar{x}) \in \mathcal{S}_{\phi_{0}}$, we have

$$
\lim _{i \rightarrow \infty} \omega_{\phi_{0}(\infty)}^{n}\left(z_{i}, x_{i}\right)=0
$$

Set $f_{i}$ to be the unique super regular disk in $\mathcal{U}_{\phi_{0}(\infty)}$ passing through the point $\left(z_{i}, x_{i}\right)$. Without loss of generality, we may assume that this sequence of disks converges to some other disk $f$. 
By definition, $\lim _{i \rightarrow \infty} \operatorname{Cap}\left(f_{i}\right)=\infty$. In other words,

$$
\lim _{i \rightarrow \infty} \int_{\Sigma}\left(\frac{\omega_{\phi_{0}(\infty)}^{n}}{\omega^{n}}\right)^{-1}\left(z_{i}, x_{i}\right)|d z|^{2}=\infty
$$

However, $\log \left(\frac{\omega_{\phi_{0}(\infty)}^{n}}{\omega^{n}}\right)$ is a sub-harmonic function with a uniform upper bound. Theorem 4.2.4 and 4.2 .13 imply that, for any compact sub-domain $\Omega \subset \Sigma^{0}$, we have

$$
\left|\triangle_{z} \log \left(\frac{\omega_{\phi_{0}(\infty)}^{n}}{\omega^{n}}\right)\right| \leq C_{\Omega}
$$

where $C_{\Omega}$ depends on $d(\partial \Omega, \partial \Sigma)$. Choose $\Omega$ so that $z_{i}, z \in \Omega$. Harnack inequality for negative harmonic functions implies that, either $\log \left(\frac{\omega_{\phi_{0}(\infty)}^{n}}{\omega^{n}}\right)$ tends to $-\infty$ simultaneously in $\Omega$ or $\log \left(\frac{\omega_{\phi_{0}(\infty)}^{n}}{\omega^{n}}\right)$ are uniformly bounded from above and below. This dichotomy holds for any compact sub-domain of $\Sigma^{0}$. For any $z \in \Sigma^{0}$ fixed, there is a unique map $w_{i}: \Sigma \rightarrow M$ such that $\left(z, w_{i}\right)$ lies in the image of $\pi \circ f_{i}$. If (passing to a subsequence if necessary)

$$
\lim _{i \rightarrow \infty}\left(\frac{\omega_{\phi_{0}(\infty)}^{n}}{\omega^{n}}\right)\left(z_{i}, x_{i}\right)=c>0
$$

then

$$
\lim _{i \rightarrow \infty}\left(\frac{\omega_{\phi_{0}(\infty)}^{n}}{\omega^{n}}\right)\left(z, w_{i}(z)\right)>0 .
$$

Following the proof of Theorem 5.3.1 and Proposition 4.2.13 we can show that in fact,

$$
\lim _{i \rightarrow \infty}\left(\frac{\omega_{\phi_{0}(\infty)}^{n}}{\omega^{n}}\right)\left(z, w_{i}(z)\right)>0, \quad \forall z \in \Sigma .
$$

The fact contradicts the assumption that the capacity of this sequence of disks blows up. Consequently, the varying volume form ratio $\left(\frac{\omega_{\phi_{0}(\infty)}^{n}}{\omega^{n}}\right)$ must converges to 0 . In other words, the volume form must be continuous in the interior of $\Sigma \times M$.

Lemma 5.4.1. Suppose $\left\{h^{(m)}, m \in \mathbb{N}\right\}$ is a sequence of continuous, positive functions in a fixed domain $\Omega$ which satisfies the following two conditions

1. The $L^{1}$ norm of $h^{(m)}$ is uniformly bounded; 
2. For any $x \in \Omega$, if $\sup _{1 \leq m \leq \infty} h^{(m)}(x)<\infty$, there exists a small neighborhood $\mathcal{O}_{x}$ of $x$ such that this sequence (pass to a a subsequence if necessary) of functions $\left\{h^{(m)}\right\}_{m=1}^{\infty}$ is uniformly continuous on $\mathcal{O}_{x}$.

Then, there exists a set $E$ of measure at most 0 and a subsequence of $\left\{h^{(m)}, m \in \mathbb{N}\right\}$ such that this subsequence is uniformly continuous on any compact subset of $\Omega \backslash E$. Moreover, there exists a limit function $h^{\infty}$ such that $\lim _{m \rightarrow \infty} h^{(m)}=h^{\infty}$ on $M \backslash E$. Moreover, $\frac{1}{1+h^{\infty}}$ is a continuous function.

Proof. By an elementary and straightforward argument.

\section{The (modified) K energy along almost solutions}

In this section, we want to prove first that the $\mathrm{K}$ energy function is sub-harmonic along any almost smooth solution of HCMA equation 1.1. One can view this as a generalization of the fact that the K energy functional is convex along a smooth geodesic. Secondly, we want to use this property of subharmonicity to prove that the (modified) $\mathrm{K}$ energy has a lower bound in any Kähler class, provided that there exists a constant scalar curvature metric (or an extremal Kähler metric) in this class.

\subsection{The Subharmonicity of the K energy}

Suppose that $\mathcal{F}_{\phi_{0}}$ is an almost super regular foliation and $\phi_{0}: \Sigma \rightarrow \overline{\mathcal{H}}$ is an almost smooth solution corresponding to it. Note that the evaluation map ev $: \Sigma \times \mathcal{M}_{\phi_{0}} \rightarrow \Sigma \times \mathcal{W}_{M}$ is smooth everywhere. The set of holomorphic disks which are not super regular has codimension at least 1 in the moduli space. Recalled that $\mathcal{V}_{\phi_{0}}=\pi \circ \operatorname{ev}\left(\Sigma \times \mathcal{U}_{\phi_{0}}\right)$. As before, set $\mathcal{S}_{\phi_{0}}=\Sigma \times M \backslash \mathcal{V}_{\phi_{0}}$. Clearly, $\mathcal{S}_{\phi_{0}}$ is a smooth sub-manifold and $\mathcal{S}_{\phi_{0}} \cap(\partial \Sigma \times M)$ has codimension at least 1 at $\partial \Sigma \times M$. We follow notations in Sections 4 in general. For convenience of the readers, let us re-state Theorem 1.3.5 here

Theorem 6.1.1. Suppose that $\phi: \Sigma \rightarrow \overline{\mathcal{H}}$ is an almost smooth solution described as in Definition 1.3.3. Then the induced $K$ energy function $\mathbf{E}: \Sigma \rightarrow \mathbb{R}($ by $\mathbf{E}(z)=\mathbf{E}(\phi(z, \cdot)))$ is weakly subharmonic and $C^{1}$ continuous (up to the boundary). More precisely,

$$
\frac{\partial^{2}}{\partial z \partial \bar{z}} \mathbf{E}(\phi(z, \cdot))=\int_{\pi \circ e v\left(z, \mathcal{U}_{\phi_{0}}\right)}\left|\mathcal{D} \frac{\partial \phi}{\partial \bar{z}}\right|_{\omega_{\phi}}^{2} \omega_{\phi}^{n} \geq 0, \quad \forall z \in \Sigma^{0}
$$

holds in $\Sigma^{0}$ in the weak sense. On $\partial \Sigma$, we have

$$
\int_{\partial \Sigma} \frac{\partial \mathbf{E}}{\partial \mathbf{n}}(\phi) d s=\int_{\pi \circ e v\left(z, \mathcal{U}_{\phi_{0}}\right)}\left|\mathcal{D} \frac{\partial \phi}{\partial \bar{z}}\right|_{\omega_{\phi}}^{2} \omega_{\phi}^{n} d s
$$


where $d s$ is the length element of $\partial \Sigma$, and $\mathbf{n}$ is the outward pointing unit normal direction at $\partial \Sigma$.

Before we proceed to the proof, let us note the following calculation scheme. For any function $f \in C^{\infty}(\Sigma \times M)$, we have

$$
\begin{aligned}
\frac{\partial}{\partial z} \int_{M} f \omega_{\phi}^{n} & =\int_{M} \frac{\partial f}{\partial z} \omega_{\phi}^{n}+\int_{M} f \triangle_{\phi} \frac{\partial \phi}{\partial z} \omega_{\phi}^{n} \\
& =\int_{M} \frac{\partial f}{\partial z} \omega_{\phi}^{n}-\int_{M} \frac{\partial f}{\partial w_{\alpha}} g_{\phi}^{\alpha \bar{\beta}} \frac{\partial^{2} \phi}{\partial z \partial w_{\bar{\beta}}} \omega_{\phi}^{n} \\
& =\int_{M} \frac{\partial f}{\partial z} \omega_{\phi}^{n}+\int_{M} \frac{\partial f}{\partial w_{\alpha}} \eta^{\alpha} \omega_{\phi}^{n} \\
& =\int_{M} \partial_{z}(f) \omega_{\phi}^{n} .
\end{aligned}
$$

Similarly, we have

$$
\frac{\partial}{\partial \bar{z}} \int_{M} f \omega_{\phi}^{n}=\int_{M} \partial_{\bar{z}}(f) \omega_{\phi}^{n}
$$

We will use these schemes throughout the proof below. We also need to use the decomposition formula of the $\mathrm{K}$ energy given in [7] (c.f. 28]). For any $\phi \in \mathcal{H}$, we have

$$
\begin{aligned}
\mathbf{E}(\phi) & =\int_{M} \ln \frac{\omega_{\phi}^{n}}{\omega_{0}^{n}} \omega^{[n]}+J(\phi)+\underline{R} I(\phi), \\
I(\phi) & =\sum_{p=0}^{n} \frac{1}{p+1} \int_{M} \phi \omega_{0}^{[n-p]} \wedge(\sqrt{-1} \partial \bar{\partial} \phi)^{[p]}, \\
J(\phi) & =-\sum_{p=0}^{n-1} \frac{1}{p+1} \int_{M} \phi \operatorname{Ric}\left(\omega_{0}\right) \wedge \omega_{0}^{[n-p-1]} \wedge(\sqrt{-1} \partial \bar{\partial} \phi)^{[p]} .
\end{aligned}
$$

Here $\underline{R}$ is the average of the scalar curvature function of any Kähler metric in $[\omega]$. Note that the component $I$ makes no contribution to the calculation of the 2nd derivatives of the K energy E. Thus, we can basically leave it aside as we calculate the second derivatives of the $K$ energy.

Proof. Let $\chi$ be any non-negative function whose support lies inside of the set $\Sigma \times M \backslash \mathcal{S}_{\phi_{0}}$. Set

$$
K_{\chi}=\int_{M} \chi \log \frac{\omega_{\phi}^{n}}{\omega_{0}^{n}} \omega_{\phi}^{[n]}
$$

Then

$$
\begin{aligned}
\frac{\partial^{2} K_{\chi}}{\partial z \partial \bar{z}}= & \int_{M} \partial_{\bar{z}} \partial_{z}\left(\chi \log \frac{\omega_{\phi}^{n}}{\omega_{0}^{n}}\right) \omega_{\phi}^{[n]} \\
= & \int_{M} \chi\left(\triangle_{z} \log \frac{\omega_{\phi}^{n}}{\omega_{0}^{n}}+\partial_{\bar{z}}\left(\left(\partial_{z} \chi\right) \log \frac{\omega_{\phi}^{n}}{\omega^{n}}\right)\right) \omega_{\phi}^{[n]}+\int_{M}\left(\partial_{z} \chi\right)\left(\log \frac{\omega_{\phi}^{n}}{\omega_{0}^{n}}\right) \omega_{\phi}^{[n]} \\
= & \int_{M} \chi\left|\mathcal{D} \frac{\partial \phi}{\partial z}\right|_{\phi}^{2} \omega_{\phi}^{[n]}+\int_{M} \chi \operatorname{Ric}\left(\omega_{0}\right) \alpha \bar{\beta} \eta^{\alpha} \eta^{\bar{\beta}} \omega_{\phi}^{[n]} \\
& \quad+\frac{\partial}{\partial \bar{z}} \int_{M}\left(\partial_{z} \chi\right) \log \frac{\omega_{\phi}^{n}}{\omega_{0}^{n}} \omega_{\phi}^{[n]}+\int_{M}\left(\partial_{z} \chi\right)\left(\partial_{\bar{z}} \log \frac{\omega_{\phi}^{n}}{\omega_{0}^{n}}\right) \omega_{\phi}^{[n]} .
\end{aligned}
$$


Let $v(z)$ be any non-negative cut off function in $\Sigma^{0}$.

$$
\begin{aligned}
\int_{\Sigma} K_{\chi} \triangle_{z} v= & \int_{\Sigma} v(z) \triangle_{z} K_{\chi} \\
= & \int_{\Sigma} v(z) \int_{M} \chi\left|\mathcal{D} \frac{\partial \phi}{\partial z}\right|_{\phi}^{2} \omega_{\phi}^{[n]}+\int_{\Sigma} v(z) \int_{M} \chi \operatorname{Ric}\left(\omega_{0}\right)_{\alpha \bar{\beta}} \eta^{\alpha} \eta^{\bar{\beta}} \omega_{\phi}^{[n]} \\
& \quad-\int_{\Sigma} \frac{\partial v}{\partial \bar{z}} \cdot \int_{M}\left(\partial_{z} \chi\right) \log \frac{\omega_{\phi}^{n}}{\omega_{0}^{n}} \omega_{\phi}^{[n]}+\int_{\Sigma} v \int_{M}\left(\partial_{z} \chi\right) \partial_{\bar{z}}\left(\frac{\omega_{\phi}^{n}}{\omega_{0}^{n}}\right) \omega_{0}^{[n]} .
\end{aligned}
$$

Consider the evaluation map:

$$
\begin{aligned}
& \sharp: \quad \Sigma \times \mathcal{M}_{\phi_{1}} \rightarrow \Sigma \times M \\
& (z, f) \rightarrow(z, \pi(f(z))) \text {. }
\end{aligned}
$$

Then, $\sharp$ is invertible on $\Sigma \times \mathcal{U}_{\phi_{0}}$. Consider any $C^{\infty}$ function $\phi \in C_{0}^{\infty}\left(\mathcal{U}_{\phi_{0}}\right) \subset C^{\infty}\left(\mathcal{M}_{\phi_{0}}\right)$ which vanishes on the boundary of $\mathcal{U}_{\phi_{0}}$. Set

$$
\chi(z, w)=\phi\left(\sharp^{-1}(z, w)\right), \quad \forall(z, w) \in \Sigma \times M .
$$

Then $\chi(z, w)$ is a smooth function in $\Sigma \times M$ whose support lies completely inside $\Sigma \times M \backslash \mathcal{S}_{\phi_{0}}$. By definition, the disk derivative $\partial_{z} \chi$ vanishes completely along super regular disks. Consequently, for any cut off function defined via formula (6.4), we have

$$
\int_{\Sigma} K_{\chi} \triangle_{z} v=\int_{\Sigma} v(z) \int_{M} \chi\left|\mathcal{D} \frac{\partial \phi}{\partial z}\right|_{\phi}^{2} \omega_{\phi}^{[n]}+\int_{\Sigma} v(z) \int_{M} \chi \operatorname{Ric}(\omega)_{\alpha \bar{\beta}} \eta^{\alpha} \eta^{\bar{\beta}} \omega_{\phi}^{[n]} .
$$

Now let $\phi$ tend to Characteristic function of $\mathcal{U}_{\phi_{0}}$ inside $\mathcal{M}_{\phi_{0}}$. Then, we have:

$$
\begin{aligned}
\int_{\Sigma} \triangle_{z} v(z) \int_{M} \log \frac{\omega_{\phi}^{n}}{\omega_{0}^{n}} \omega_{\phi}^{[n]} & =\int_{\Sigma \times M \backslash \mathcal{S}_{\phi_{0}}} \triangle_{z} v(z) \log \frac{\omega_{\phi}^{n}}{\omega_{0}^{n}} \omega_{\phi}^{[n]} \\
& =\int_{\Sigma \times M \backslash \mathcal{S}_{\phi_{0}}} v(z)\left|\mathcal{D} \frac{\partial \phi}{\partial z}\right|_{\phi}^{2} \omega_{\phi}^{[n]}+\int_{\Sigma \times M \backslash \mathcal{S}_{\phi_{0}}} v(z) \operatorname{Ric}(\omega)_{\alpha \bar{\beta}} \eta^{\alpha} \eta^{\bar{\beta}} \omega_{\phi}^{[n]} \\
& =\int_{\Sigma \times M \backslash \mathcal{S}_{\phi_{0}}} v(z)\left|\mathcal{D} \frac{\partial \phi}{\partial z}\right|_{\phi}^{2} \omega_{\phi}^{[n]}+\int_{\Sigma \times M} v(z) \operatorname{Ric}(\omega)_{\alpha \bar{\beta}} \eta^{\alpha} \eta^{\bar{\beta}} \omega_{\phi}^{[n]} .
\end{aligned}
$$

The first and the last equality holds because that

$$
\log \frac{\omega_{\phi}^{n}}{\omega_{0}^{n}} \frac{\omega_{\phi}^{n}}{\omega_{0}^{n}} \quad \text { and } \quad \operatorname{Ric}\left(\omega_{0}\right)_{\alpha \bar{\beta}} \eta^{\alpha} \eta^{\bar{\beta}} \frac{\omega_{\phi}^{n}}{\omega_{0}^{n}}
$$

both vanish on $\mathcal{S}_{\phi_{0}}$. On the other hand,

$$
\begin{aligned}
\frac{\partial^{2} J(\phi)}{\partial z \partial \bar{z}}= & -\int_{M} \frac{\partial^{2} \phi}{\partial z \partial \bar{z}} \operatorname{Ric}(\omega) \wedge \omega_{\phi}^{[n-1]}-\int_{M} \frac{\partial \phi}{\partial z} \operatorname{Ric}\left(\omega_{0}\right) \wedge \sqrt{-1} \partial \bar{\partial} \frac{\partial \phi}{\partial \bar{z}} \wedge \omega_{\phi}^{[n-2]} \\
= & -\int_{M} \frac{\partial^{2} \phi}{\partial z \bar{z}} \operatorname{Ric}\left(\omega_{0}\right) \wedge \omega_{\phi}^{[n-1]}+\int_{M} \operatorname{Ric}\left(\omega_{0}\right) \wedge \sqrt{-1} \partial \frac{\partial \phi}{\partial \bar{z}} \wedge \bar{\partial} \frac{\partial \phi}{\partial z} \wedge \omega_{\phi}^{[n-2]} \\
= & -\int_{M} \frac{\partial^{2} \phi}{\partial z \partial \bar{z}} \operatorname{Ric}\left(\omega_{0}\right) \wedge \omega_{\phi}^{[n-1]} \\
& \quad+\int_{M}\left(g_{\phi}^{\alpha \bar{\beta}} \operatorname{Ric}\left(\omega_{0}\right)_{\alpha \bar{\beta}} \cdot g_{\phi}^{r \bar{\delta}} \frac{\partial^{2} \phi}{\partial \bar{z} \partial w_{r}} \frac{\partial^{2} \phi}{\partial z \partial w_{\bar{\delta}}}-\operatorname{Ric}\left(\omega_{0}\right)_{\alpha \bar{\beta}} \eta^{\alpha} \eta^{\bar{\beta}}\right) \omega^{[n]} \\
= & -\int_{M}\left(\frac{\partial^{2} \phi}{\partial z \partial \bar{z}}-g_{\phi}^{r \bar{\delta}} \eta^{\alpha} \eta^{\bar{\beta}}\right) \operatorname{Ric}\left(\omega_{0}\right) \wedge \omega_{\phi}^{[n-1]}-\int_{M} \operatorname{Ric}\left(\omega_{0}\right)_{\alpha \bar{\beta}} \eta^{\alpha} \eta^{\bar{\beta}} \omega^{[n]} \\
= & -\int_{M} \operatorname{Ric}\left(\omega_{0}\right)_{\alpha \bar{\beta}} \eta^{\alpha} \eta^{\bar{\beta}} \omega^{[n]} .
\end{aligned}
$$


The last equality holds since $\phi$ is a solution to the Homogenous complex Monge-Ampere equation. Therefore,

$$
\int_{\Sigma} \triangle_{z} v J(\phi)=-\int_{\Sigma} \triangle_{z} v \int_{M} \operatorname{Ric}\left(\omega_{0}\right)_{\alpha \bar{\beta}} \eta^{\alpha} \eta^{\bar{\beta}} \omega^{[n]}
$$

Using the decomposition formula for K energy (6.1), we have

$$
\int_{\Sigma}\left(\triangle_{z} v(z)\right) \mathbf{E}(\phi(z, \cdot))=\int_{\Sigma \times M \backslash \mathcal{S}_{\phi_{0}}} v(z)\left|\mathcal{D} \frac{\partial \phi}{\partial z}\right|_{\phi}^{2} \omega_{\phi}^{[n]} \geq 0
$$

This implies that the K energy functional is sub-harmonic in $\Sigma^{0}$. Next we want to derive a formula for the first derivative of the K energy. For any $v \in C_{0}^{\infty}(\Sigma)$, we have

$$
\begin{aligned}
\int_{\Sigma} \frac{\partial v(z)}{\partial z} \cdot K_{\chi} & =\int_{\Sigma} v(z) \cdot \frac{\partial}{\partial z} K_{\chi} \\
& =\int_{\Sigma} v(z)\left(\int_{M} \partial_{z}(\chi) \log \frac{\omega_{\phi}^{n}}{\omega_{0}^{n}} \omega_{\phi}^{[n]}+\int_{M} \chi \partial_{z}\left(\frac{\omega_{\phi}^{n}}{\omega_{0}^{n}}\right) \omega^{[n]}\right) .
\end{aligned}
$$

For any small $\delta>0$, let $\Sigma_{\delta}=\{z \in \Sigma:|z| \leq 1-\delta\}$. Since $v(z)$ is an arbitrary compactly supported function in $\Sigma^{0}$, we obtain

$$
\int_{\partial \Sigma_{\delta}} \zeta(z) \frac{\partial}{\partial z} K_{\chi}=\int_{\partial \Sigma_{\delta}} \zeta(z)\left(\int_{M} \partial_{z}(\chi) \log \frac{\omega_{\phi}^{n}}{\omega^{n}} \omega_{\phi}^{[n]}+\int_{M} \chi \partial_{z}\left(\frac{\omega_{\phi}^{n}}{\omega_{0}^{n}}\right) \omega_{0}^{[n]}\right)
$$

where $\zeta$ is any smooth function.

Now let $\chi$ tend to the Characteristic function of $\Sigma \times M \backslash \mathcal{S}_{\phi_{0}}$. As before, the first term in the right hand side vanishes, we have

$$
\int_{\partial \Sigma_{\delta}} \zeta(z) \frac{\partial}{\partial z} \int_{M} \log \frac{\omega_{\phi}^{n}}{\omega_{0}^{n}} \omega_{\phi}^{[n]}=\int_{\partial \Sigma_{\delta}} \zeta(z) \int_{M \backslash \mathcal{S}_{\phi_{0}}} \partial_{z}\left(\frac{\omega_{\phi}^{n}}{\omega_{0}^{n}}\right) \omega_{0}^{[n]} .
$$

For the K energy, we have

$$
\begin{aligned}
\int_{\partial \Sigma_{\delta}} \zeta(z) \frac{\partial}{\partial z} \mathbf{E}(\phi(z, \cdot))= & \int_{\partial \Sigma_{\delta}} \zeta(z) \int_{M \backslash \mathcal{S}_{\phi_{0}}} \partial_{z}\left(\frac{\omega_{\phi}^{n}}{\omega_{0}^{n}}\right) \omega_{0}^{n} \\
& -\int_{\partial \Sigma_{\delta}} \zeta(z) \int_{M} \frac{\partial \phi}{\partial z}\left(\operatorname{Ric}\left(\omega_{0}\right)-\underline{R} \omega_{\phi}\right) \wedge \omega_{\phi}^{[n-1]}
\end{aligned}
$$

Integrating by parts on the left hand side of equation (6.5) and letting $v$ approach the characterstic function of $\Sigma_{\delta}$, we obtain

$$
\begin{aligned}
\int_{\Sigma_{\delta} \times M \backslash \mathcal{S}_{\phi_{0}}}\left|\mathcal{D} \frac{\partial \phi}{\partial z}\right|_{\phi}^{2} \omega_{\phi}^{[n]} & =\int_{\partial \Sigma_{\delta}} \frac{\bar{z}}{|z|} \frac{\partial}{\partial z} \mathbf{E}(\phi(z, \cdot)) \\
& =\int_{\partial \Sigma_{\delta}} \frac{\bar{z}}{|z|} \int_{M \backslash \mathcal{S}} \partial_{z}\left(\frac{\omega_{\phi}^{n}}{\omega_{0}^{n}}\right) \omega_{0}^{n}-\int_{\Sigma_{\delta}} \frac{\bar{z}}{|z|} \int_{M} \frac{\partial \phi}{\partial z}\left(\operatorname{Ric}\left(\omega_{0}\right)-\underline{R} \omega_{\phi}\right) \wedge \omega_{\phi}^{[n-1]} .
\end{aligned}
$$


The first and the third terms in the formula above, are both integration on $\pi \circ \operatorname{ev}\left(\Sigma \times \mathcal{U}_{\phi_{0}}\right)$ where Kähler metric is smooth. Therefore, taking limit as $\delta \rightarrow 0$, we arrive

$$
\int_{\Sigma \times M \backslash \mathcal{S}_{\phi_{0}}}\left|\mathcal{D} \frac{\partial \phi}{\partial z}\right|_{\phi}^{2} \omega_{\phi}^{[n]}=\int_{\partial \Sigma} \frac{\bar{z}}{|z|} \int_{M \backslash \mathcal{S}_{\phi_{0}}} \partial_{z}\left(\frac{\omega_{\phi}^{n}}{\omega_{0}^{n}}\right) \omega_{0}^{n}-\int_{\partial \Sigma} \frac{\bar{z}}{|z|} \int_{M} \frac{\partial \phi}{\partial z}\left(\operatorname{Ric}\left(\omega_{0}\right)-\underline{R} \omega_{\phi}\right) \wedge \omega_{\phi}^{[n-1]} .
$$

In the above process of taking the limit, the only term which needs special attentions is:

$$
\lim _{z \rightarrow z_{0} \in \partial \Sigma} \int_{\{z\} \times M \backslash \mathcal{S}_{\phi_{0}}} \partial_{z}\left(\frac{\omega_{\phi}^{n}}{\omega_{0}^{n}}\right) \omega_{0}^{n}=\int_{\left\{z_{0}\right\} \times M \backslash \mathcal{S}_{\phi_{0}}} \partial_{z}\left(\frac{\omega_{\phi}^{n}}{\omega_{0}^{n}}\right) \omega_{0}^{n} .
$$

This is equivalent to say that the $z$ - derivative of the $\mathrm{K}$ energy is continuous as $z \rightarrow z_{0} \in$ $\partial \Sigma\left(|z|<1=\left|z_{0}\right|, \forall z_{0} \in \partial \Sigma\right)$. For any $\delta>0$ fixed, choose any $\delta$ neighborhood of the set of non-super regular disks (Denoted by $E_{\delta} \subset \mathcal{M}_{\phi_{0}}$ ) such that

$$
\lim _{\delta \rightarrow 0} \operatorname{mes}\left(E_{\delta}\right)=0 .
$$

Set

$$
\mathcal{S}_{\delta}=\sharp\left(\Sigma \times E_{\delta}\right) .
$$

Let $\left(t_{1}, t_{2}, \cdots t_{2 n}\right)$ be the coordinate variables in $\mathcal{M}_{\phi_{0}}$ and $w_{1}, w_{2}, \cdots w_{n}$ be the complex coordinate variables in $M$. Set

$$
J=\left(\frac{\partial w_{\alpha}}{\partial t_{i}} \frac{\partial w_{\bar{\beta}}}{\partial t_{i}}\right)
$$

as the Jacobi matrix. Then, $J$ is a smooth complex matrix valued function in $\mathcal{M}$, and invertible at $\mathcal{M}_{\phi_{0}}$. Denote by $\Gamma\left(\omega_{0}\right)$ the connection form of the Kähler metric which corresponds to the Kähler form $\omega_{0}$. In the following calculation, we take covariant derivatives with respect to $\omega_{0}$.

Clearly,

$$
\lim _{z \rightarrow z_{0} \in \partial \Sigma} \int_{\{z\} \times M \backslash \mathcal{S}_{\delta}} \partial_{z}\left(\frac{\omega_{\phi}^{n}}{\omega_{0}^{n}}\right) \omega_{0}^{n}=\int_{\left\{z_{0}\right\} \times M \backslash \mathcal{S}_{\delta}} \partial_{z}\left(\frac{\omega_{\phi}^{n}}{\omega_{0}^{n}}\right) \omega_{0}^{n} .
$$

Now we need to show that the remaining portion is $o(\delta)$.

$$
\begin{aligned}
\lim _{z \rightarrow z_{0} \in \partial \Sigma} \int_{\{z\} \times \mathcal{S}_{\delta} \backslash \mathcal{S}_{\phi_{0}}} \partial_{z}\left(\frac{\omega_{\phi}^{n}}{\omega_{0}^{n}}\right) \omega_{0}^{n}= & \int_{\left\{z_{0}\right\} \times \mathcal{S}_{\delta} \backslash \mathcal{S}} \eta^{\alpha}{ }_{, w_{\alpha}}\left(\omega_{0}\right)\left(\frac{\omega_{\phi}^{n}}{\omega_{0}^{n}}\right) \omega_{0}^{n} \\
= & \int_{\left\{z_{0}\right\} \times \mathcal{S}_{\delta} \backslash \mathcal{S}}\left(\frac{\partial \eta^{\alpha}}{\partial w_{\alpha}}-\Gamma_{\beta \alpha}^{\alpha}\left(\omega_{0}\right) \eta^{\beta}\right)\left(\frac{\omega_{\phi}^{n}}{\omega_{0}^{n}}\right) \omega^{n} \\
= & \int_{\left\{z_{0}\right\} \times\left(E_{\delta} \cap \mathcal{U}_{\phi_{0}}\right)}\left(\frac{\partial \eta^{\alpha}}{\partial w_{\alpha}}-\Gamma_{\beta \alpha}^{\alpha}\left(\omega_{0}\right) \eta^{\beta}\right)\left(\frac{\omega_{\phi}^{n}}{\omega_{0}^{n}}\right) \operatorname{det} g_{\alpha \bar{\beta}} \operatorname{det}(J) d t \\
= & \int_{\left\{z_{0}\right\} \times\left(E_{\delta} \cap \mathcal{U}_{\phi_{0}}\right)} \frac{\partial \eta^{\alpha}}{\partial x_{k}}\left(\frac{\partial x_{k}}{\partial w_{\alpha}} \operatorname{det}(J)\right) \operatorname{det} g_{\alpha \bar{\beta}} d t \\
& \quad-\int_{\left\{z_{0}\right\} \times\left(E_{\delta} \cap \mathcal{U}_{\phi_{0}}\right)} \Gamma_{\beta \alpha}^{\alpha}\left(\omega_{0}\right) \eta^{\beta}\left(\frac{\omega_{\phi}^{n}}{\omega_{0}^{n}}\right) \operatorname{det} g_{\alpha \bar{\beta}} \operatorname{det}(J) d t \rightarrow 0
\end{aligned}
$$


as $\delta \rightarrow 0$. This is because all terms in the last formula are uniformly bounded and the measure of $E_{\delta}$ tends to 0 as $\delta \rightarrow 0$. Here

$$
d t=d t^{1} d t^{2} \cdots d t^{2 n}
$$

Consequently, we have shown that

$$
\int_{\Sigma \times M \backslash \mathcal{S}_{\phi_{0}}}\left|\mathcal{D} \frac{\partial \phi}{\partial z}\right|_{\phi}^{2} \omega_{\phi}^{[n]}=\int_{\partial \Sigma} \frac{\bar{z}}{|z|} \int_{M \backslash \mathcal{S}_{\phi_{0}}} \partial_{z}\left(\frac{\omega_{\phi}^{n}}{\omega_{0}^{n}}\right) \omega_{0}^{n}-\int_{\partial \Sigma} \frac{\bar{z}}{|z|} \int_{M} \frac{\partial \phi}{\partial z}\left(\operatorname{Ric}\left(\omega_{0}\right)-\underline{R} \omega_{\phi}\right) \wedge \omega_{\phi}^{[n-1]} .
$$

In other words, we have

$$
\begin{aligned}
\int_{\Sigma \times M \backslash \mathcal{S}_{\phi_{0}}}\left|\mathcal{D} \frac{\partial \phi}{\partial z}\right|_{\phi}^{2} \omega_{\phi}^{[n]} & =\int_{\partial \Sigma} \frac{\bar{z}}{|z|} \frac{\partial}{\partial z} \mathbf{E}(\phi(z, \cdot)) \\
& =\int_{\partial \Sigma} \frac{\partial E}{\partial \mathbf{n}} .
\end{aligned} .
$$

The theorem is then proved.

If we replace the almost smooth solution by a partially smooth solution, then

Corollary 6.1.2. Suppose that $\phi: \Sigma \rightarrow \overline{\mathcal{H}}$ is a partially smooth solution described as in Definition 1.3.1. Then the induced $K$ energy function $\mathbf{E}: \Sigma \rightarrow \mathbf{R}($ by $\mathbf{E}(z)=\mathbf{E}(\phi(z, \cdot)))$ is a bounded weakly sub-harmonic function in $\Sigma$ such that

$$
\frac{\partial^{2}}{\partial z \partial \bar{z}} \mathbf{E}(\phi(z, \cdot)) \geq \int_{M \backslash \mathcal{S}_{\phi_{0}}}\left|\mathcal{D} \frac{\partial \phi}{\partial \bar{z}}\right|_{\omega_{\phi}}^{2} \omega_{\phi}^{n} \geq 0
$$

holds in $\Sigma$ in the weak sense. Moreover,

$$
\int_{\partial \Sigma} \frac{\partial \mathbf{E}}{\partial \mathbf{n}}(\phi(z, \cdot)) d s \geq \int_{\Sigma \times M \backslash \mathcal{S}_{\phi_{0}}}\left|\mathcal{D} \frac{\partial \phi}{\partial \bar{z}}\right|_{\omega_{\phi}}^{2} \omega_{\phi}^{n} d s
$$

where $d s$ is the length element of $\partial \Sigma$, and $\mathbf{n}$ is the outward pointing unit normal direction at $\partial \Sigma$.

In the case of extremal Kähler metrics, E. Calabi showed [5] that any extremal Kähler metric must be invariant under some maximal compact subgroup of the automorphism group. Consider all the metrics which are symmetric under the same maximal connected compact subgroup of the automorphism group. According to 15, there exists a unique extremal holomorphic vector field

$$
Y=Y^{\alpha} \frac{\partial}{\partial w_{\alpha}}
$$

which is the gradient vector field of scalar curvature if the metric is extremal. Note that this vector filed is unique in each Kähler class. Consider

$$
\mathcal{L}_{Y} \omega_{\phi}=\sqrt{-1} \partial \bar{\partial} \theta(\phi)
$$


Here $\theta(\phi)$ is a real valued potential function for $\phi$. It is well known that one can modify the definition of the $\mathrm{K}$ energy by this potential function such that the critical point of the new functional is the extremal Kähler metric. Set

$$
\begin{aligned}
\frac{d \tilde{\mathbf{E}}}{d t}(\phi(t)) & =-\int_{M}(R(\phi)-\underline{R}-\theta(\phi)) \frac{\partial \phi}{\partial t} \omega_{\phi}^{[n]} \\
& =\frac{d \mathbf{E}}{d t}(\phi(t))+\int_{M} \theta(\phi) \frac{\partial \phi}{\partial t} \omega_{\phi}^{[n]} .
\end{aligned}
$$

Here $\theta(\phi)$ is the real valued potential function for this holomorphic vector field. This is true when all Kähler potentials are invariant under the maximal compact subgroup.

$$
\frac{\partial \theta(\phi)}{\partial w_{\alpha}}=Y^{\bar{\beta}} g_{\phi, \alpha \bar{\beta}}
$$

It is easy to see that

$$
\frac{\partial \theta}{\partial t}=Y\left(\frac{\partial \phi}{\partial t}\right)=g_{\phi}^{\alpha \bar{\beta}} \frac{\partial \theta}{\partial w_{\alpha}} \frac{\partial^{2} \phi}{\partial t \partial w_{\bar{\beta}}}
$$

Then,

$$
\begin{aligned}
\frac{d}{d t} \int_{M} \theta(\phi) \frac{\partial \phi}{\partial t} \omega_{\phi}^{[n]} & =\int_{M}\left(\frac{\partial \theta}{\partial t} \frac{\partial \phi}{\partial t}+\theta \frac{\partial^{2} \phi}{\partial t^{2}}+\theta \frac{\partial \phi}{\partial t} \triangle_{\phi}\left(\frac{\partial \phi}{\partial t}\right)\right) \omega_{\phi}^{[n]} \\
& =\int_{M}\left(g_{\phi}^{\alpha \bar{\beta}} \frac{\partial \theta}{\partial w_{\alpha}} \frac{\partial^{2} \phi}{\partial t \partial w_{\bar{\beta}}} \frac{\partial \phi}{\partial t}+\theta \frac{\partial^{2} \phi}{\partial t^{2}}+\theta \frac{\partial \phi}{\partial t} \triangle_{\phi}\left(\frac{\partial \phi}{\partial t}\right)\right) \omega_{\phi}^{[n]} \\
& =\int_{M}\left(-\theta \triangle_{\phi}\left(\frac{\partial \phi}{\partial t}\right)-\theta \frac{1}{2}\left|\nabla \frac{\partial \phi}{\partial t}\right|_{\phi}^{2}+\theta \frac{\partial^{2} \phi}{\partial t^{2}}+\theta \frac{\partial \phi}{\partial t} \triangle_{\phi}\left(\frac{\partial \phi}{\partial t}\right)\right) \omega_{\phi}^{[n]} \\
& =\int_{M} \theta(\phi)\left(\frac{\partial^{2} \phi}{\partial t^{2}}-\frac{1}{2}\left|\nabla \frac{\partial \phi}{\partial t}\right|_{\phi}^{2}\right) \omega_{\phi}^{[n]}
\end{aligned}
$$

By a similar calculation, we obtain

$$
\frac{\partial^{2} \tilde{\mathbf{E}}}{\partial z \partial \bar{z}}=\frac{\partial^{2} \mathbf{E}}{\partial z \partial \bar{z}}+\int_{M}\left(\frac{\partial^{2} \phi}{\partial z \partial \bar{z}}-\frac{1}{2}\left|\nabla \frac{\partial \phi}{\partial z}\right|_{\phi}^{2}\right) \theta(\phi(\cdot, z)) \omega_{\phi}^{[n]} .
$$

For an almost smooth solution to HCMA equation (1.1), the second term vanishes completely. Note that if $\omega_{\phi}$ is uniformly bounded from above, then $\theta(\phi)$ is uniformly Lipschitz. This is a key technical step in generalizing Theorem 6.1.1 to the case of extremal Kähler metrics. Similarly, the same approximating proof will will work in this case as well. Thus ${ }^{22}$.

Corollary 6.1.3. For any partially smooth solution $\phi \in \overline{\mathcal{H}}$ (c.f. Definition 1.3.1) which is invariant under the maximal compact subgroup, we have

$$
\int_{M \times \Sigma \backslash \mathcal{S}_{\phi_{0}}}\left|\mathcal{D} \frac{\partial \phi}{\partial z}\right|_{\omega_{\phi}}^{2} \omega_{\phi}^{n} d \tau d \bar{z} \leq \int_{\partial \Sigma} \frac{\partial \tilde{\mathbf{E}}}{\partial \mathbf{n}}(\phi) d s,
$$

where the left hand side is evaluated at points where Kähler metric is smooth. Equality holds for any partially smooth solution. Moreover, $\tilde{E}$ is a bounded weakly sub-harmonic function on $\Sigma$.

\footnotetext{
${ }^{22}$ The crucial point is that $\theta(\phi(z, \cdot))$ is uniformly Lipschitz.
} 


\subsection{The lower bound of the (modified) K energy}

In this subsection, we want to use Theorem 6.1.1 and Theorem 6.1.2 to establish a lower bound for the (modified) K energy. In Sections 6-8, for simplicity, we always deal with constant scalar curvature metric and $\mathrm{K}$ energy functional. The corresponding treatment of extremal Kähler metric is parallel and we leave for interested readers. Now we set up some notations here first.

For any two Kähler potentials $\phi_{0}, \phi_{1} \in \mathcal{H}$, we want to use almost smooth solution to approximate the $C^{1,1}$ geodesic between $\phi_{0}$ and $\phi_{1}$. For any integer $l$, consider Drichelet problem for HCMA equation 1.1 on the rectangle domain $\Sigma_{l}=[-l, l] \times[0,1]$ with boundary value as

$$
\phi(s, 0)=\phi_{0}, \phi(s, 1)=\phi_{1} ; \quad \phi( \pm l, t)=(1-t) \phi_{0}+(1-t) \phi_{1}, \quad(s, t) \in \Sigma_{l} .
$$

We may modify this boundary map in the four corners so that the domain is smooth without corner. Denote the almost smooth solution by $\phi^{(l)}: \Sigma_{l} \rightarrow \mathcal{H}$ which corresponds to this boundary map $^{23}$. According to [8], $\phi^{(l)}$ has a uniform $C^{1,1}$ upper bound which is independent of $l$. Set

$$
\mathbf{E}^{(l)}(s, t)=\mathbf{E}\left(\phi^{(l)}(s, t)\right), \quad \forall(s, t) \in \Sigma_{l} .
$$

Then, $E^{(l)}$ is a sequence of weakly sub-harmonic function with uniform bound ${ }^{24}$ such that

$$
\mathbf{E}^{(l)}(s, 0)=\mathbf{E}\left(\phi_{0}\right)=A, \quad \text { and } \mathbf{E}^{(l)}(s, 1)=\mathbf{E}\left(\phi_{1}\right)=B .
$$

Now we are ready to prove (cf. Theorem 1.1.2)

Theorem 6.2.1. Any extremal Kähler metric realizes the absolute minimum of the modified $K$ energy. Furthermore, the Kähler class is K-semistable if it admits a constant scalar curvature metric.

Proof. We give a detailed proof for the case of constant scalar curvature metric. The more general extremal Kähler metric case is similar and we leave it for interested readers. Suppose that $\phi_{0}$ is a constant scalar curvature metric. Then $\frac{\partial \mathbf{E}^{(l)}}{\partial t}=\frac{\partial \mathbf{E}^{(l)}}{\partial s}=0$ when $t=0$. Our theorem is reduced to the following claim:

Claim: $B=\mathbf{E}\left(\phi_{1}\right) \geq \mathbf{E}\left(\phi_{0}\right)=A$.

Let $\kappa:(-\infty, \infty) \rightarrow \mathbf{R}$ be a smooth non-negative function such that $\kappa \equiv 1$ on $\left[-\frac{1}{2}, \frac{1}{2}\right]$ and vanishes outside of $\left[-\frac{3}{4}, \frac{3}{4}\right]$. Set

$$
\kappa^{(l)}(s)=\frac{1}{v} \kappa\left(\frac{s}{l}\right), \quad \text { where } v=\int_{-\infty}^{\infty} \kappa(s) d s .
$$

\footnotetext{
${ }^{23}$ We may need to alter the boundary value slightly.

${ }^{24}$ because the decomposition formula for K energy (6.1) and the uniform $C^{1,1}$ bound on $\phi^{(l)}$.
} 
Set

$$
f^{(l)}(t)=\int_{-\infty}^{\infty} \kappa^{(l)}(s) \mathbf{E}^{(l)}(s, t) d s .
$$

Then

$$
\begin{aligned}
f^{(l)}(0) & =\int_{-\infty}^{\infty} \kappa^{(l)}(s) \mathbf{E}^{(l)}(s, 0) d s=\int_{-\infty}^{\infty} \kappa^{(l)}(s) A d s=A, \\
f^{(l)}(1) & =\int_{-\infty}^{\infty} \kappa^{(l)}(s) \mathbf{E}^{(l)}(s, 1) d s=\int_{-\infty}^{\infty} \kappa^{(l)}(s) B d s=B, \\
\left.\frac{d f^{(l)}}{d t}\right|_{t=0} & =0 .
\end{aligned}
$$

Now

$$
\begin{aligned}
\mathbf{E}\left(\phi_{1}\right)-\mathbf{E}\left(\phi_{0}\right) & =\left.f^{(l)}(t)\right|_{0} ^{1} \\
& =\int_{0}^{1} \int_{0}^{\theta} \frac{d^{2} f^{(l)}}{d t^{2}} d t d \theta \\
& =\int_{0}^{1} \int_{0}^{\theta} \int_{-\infty}^{\infty} \kappa^{(l)}(s) \frac{\partial^{2} \mathbf{E}^{(l)}}{\partial t^{2}} d s d t d \theta \\
& =\int_{0}^{1} \int_{0}^{\theta} \int_{-\infty}^{\infty} \kappa^{(l)}(s) \triangle_{s, t} \mathbf{E}^{(l)} d s d t d \theta-\int_{0}^{1} \int_{0}^{\theta} \int_{-\infty}^{\infty} \kappa^{(l)}(s) \frac{\partial^{2} \mathbf{E}^{(l)}}{\partial s^{2}} d s d t d \theta \\
& \geq-\int_{0}^{1} \int_{0}^{\theta} \int_{-\infty}^{\infty} \kappa^{(l)}(s) \frac{\partial^{2} \mathbf{E}^{(l)}}{\partial s^{2}} d s d t d \theta \\
& =-\int_{0}^{1} \int_{0}^{\theta} \int_{-\infty}^{\infty} \frac{d^{2} \kappa^{(l)}(s)}{d s^{2}} \mathbf{E}^{(l)}(s, t) d s d t d \theta \\
& =-\left.\frac{1}{l^{2}} \frac{1}{v} \int_{0}^{1} \int_{0}^{\theta} \int_{-\infty}^{\infty} \frac{d^{2} \kappa^{(l)}}{d s^{2}}\right|_{\frac{s}{l}} \mathbf{E}^{(l)}(s, t) d s d t d \theta
\end{aligned}
$$

Note that $\left|\mathbf{E}^{(l)}(s, t)\right|$ has a unform bound $C$. Thus,

$$
\begin{aligned}
\frac{1}{l^{2}} \frac{1}{v}\left|\int_{-\infty}^{\infty} \frac{d^{2} \kappa^{(l)}}{d s^{2}}\right|_{\frac{s}{l}} \mathbf{E}^{(l)}(s, t) d s \mid & \leq \frac{1}{l^{2}} \frac{1}{v} \int_{-\infty}^{\infty}\left|\frac{d^{2} \kappa^{(l)}}{d s^{2}}\right|_{\frac{s}{l}} \mid \mathbf{E}^{(l)}(s, t) d s \\
& \leq \frac{C}{v l^{2}} \int_{-\infty}^{\infty}\left|\frac{d^{2} \kappa^{(l)}}{d s^{2}}\right|_{\frac{s}{l}} \mid d s \\
& =\left.\frac{C}{v l} \int_{-\infty}^{\infty}\left|\frac{d^{2} \kappa^{(l)}}{d s^{2}}\right|_{s}\left|d s=\frac{C}{v l} \int_{-1}^{1}\right| \frac{d^{2} \kappa^{(l)}}{d s^{2}}\right|_{s} \mid d s \\
& \leq \frac{C}{l}
\end{aligned}
$$


for some uniform constant $C$. Therefore, we have

$$
\begin{aligned}
\mathbf{E}\left(\phi_{1}\right)-\mathbf{E}\left(\phi_{0}\right) & \geq-\left.\frac{1}{l^{2}} \frac{1}{v} \int_{0}^{1} \int_{0}^{\theta} \int_{-\infty}^{\infty} \frac{d^{2} \kappa}{d s^{2}}\right|_{\frac{s}{l}} \mathbf{E}^{(l)}(s, t) d s d t d \theta \\
& \geq-\int_{0}^{1} \int_{0}^{\theta} \frac{1}{l^{2}} \frac{1}{v}\left|\int_{-\infty}^{\infty} \frac{d^{2} \kappa}{d s^{2}}\right|_{\frac{s}{l}} \mathbf{E}^{(l)}(s, t) d s \mid d t d \theta \\
& \geq-\int_{0}^{1} \int_{0}^{\theta} \frac{C}{l} d t d \theta=-\frac{C}{2 l}
\end{aligned}
$$

As $l \rightarrow \infty$, we have

$$
\mathbf{E}\left(\phi_{1}\right) \geq \mathbf{E}\left(\phi_{0}\right)
$$

Since $\phi_{1}$ is an arbitrary Kähler potential, the theorem is then proved.

\section{$7 \quad$ Partial regularity of the K energy minimzer}

\subsection{Strong convergence lemma for volume form}

In this subsection, we want to prove

Theorem 7.1.1. Suppose that $\left\{\phi_{m}, m \in \mathbb{N}\right\}$ is a sequence of Kähler potentials in $\mathcal{H}$ with uniform $C^{1,1}$ bound and suppose that $\phi_{m} \rightarrow \phi_{0} \in \overline{\mathcal{H}}$ strongly in $C^{1, \alpha}(\forall \alpha<1)$ and weakly in $W^{2, p}$ for $p$ large enough. If the corresponding sequence of $K$ energies $\left\{\mathbf{E}\left(\phi_{m}\right), m \in \mathbb{N}\right\}$ is a cauchy sequence and

$$
\lim _{l \rightarrow \infty} \mathbf{E}\left(\phi_{l}\right) \leq \mathbf{E}\left(\phi_{0}\right)
$$

then $\frac{\omega_{\phi_{m}}^{n}}{\omega^{n}}$ converges strongly to $\frac{\omega_{\phi_{0}}^{n}}{\omega^{n}}$ in $L^{2}(M, \omega)$.

Proof. Set $f_{m}=\frac{\omega_{\phi_{m}}^{n}}{\omega^{n}} \leq C$ and $g=\frac{\omega_{\phi_{0}}^{n}}{\omega^{n}}$. Applying the decomposition formula of the $\mathrm{K}$ energy (6.1), we obtain that $\left\{\int_{M} f_{m} \log f_{m}, m \in \mathbb{N}\right\}$ is a Cauchy sequence.

Since $\left\{\phi_{l}, l \in \mathbb{N}\right\}$ weakly converges to $\phi_{0}$ in the $W^{2, p}$ norm for $p$ large enough, the lower order part of the $\mathrm{K}$ energy converges to the corresponding lower order part of the $\mathrm{K}$ energy of $\phi_{0}$. Thus

$$
\int_{M} f_{l} \log f_{l} \omega^{n}-\int_{M} g \log g=\mathbf{E}\left(\phi_{l}\right)-\mathbf{E}\left(\phi_{0}\right)+o\left(\frac{1}{l}\right) \leq o\left(\frac{1}{l}\right) .
$$

Define $F(u)=u \log u$. For any $l$ large enough and for any $\epsilon>0$, set $F(t)=F\left(t f_{l}+(1-t)(g+\right.$ $\epsilon))=F(a t+b)$ where

$$
a=f_{l}-g-\epsilon, \quad \text { and } \quad b=g+\epsilon .
$$

Note that $a, b$ are both functions in $M$. Clearly, we have

$$
|a|+|b| \leq C
$$


Note that

$$
F^{\prime}(t)=a \log (a t+b)+a,
$$

and

$$
F^{\prime \prime}(t)=\frac{a^{2}}{a t+b} \geq \frac{a^{2}}{C}, \quad \forall t \in[0,1] .
$$

Thus,

$$
\begin{aligned}
\int_{M} F^{\prime}(0) \omega^{n} & =\int_{M}(a \log b+a) \omega^{n} \\
& =\int_{M}\left(f_{l}-g-\epsilon\right) \log \left(g_{m}+\epsilon\right) \omega^{n}+\int_{M}\left(f_{l}-g-\epsilon\right) \omega^{n} \\
& =\int_{M}\left(f_{l}-g-\epsilon\right) \log (g+\epsilon) \omega^{n}+\int_{M} \omega_{\phi_{l}}^{n}-\int_{M} \omega_{\phi_{0}}^{n}-\epsilon \operatorname{vol}(M) \\
& =\int_{M}\left(f_{l}-g-\epsilon\right) \log (g+\epsilon) \omega^{n}-o(\epsilon) .
\end{aligned}
$$

Taking the following double limits

$$
\begin{aligned}
\lim _{\epsilon \rightarrow 0} \lim _{l \rightarrow \infty} \int_{M} F^{\prime}(0) \omega^{n} & =\lim _{\epsilon \rightarrow 0} \lim _{l \rightarrow \infty}\left(\int_{M}\left(f_{l}-g-\epsilon\right) \log (g+\epsilon) \omega^{n}-o(\epsilon)\right) \\
& =\lim _{\epsilon \rightarrow 0}\left(\int_{M}(g-g-\epsilon) \log (g+\epsilon) \omega^{n}-o(\epsilon)\right) \\
& =\lim _{\epsilon \rightarrow 0} o(\epsilon)=0 .
\end{aligned}
$$

The second equality used the fact that $f_{l} \rightarrow g$ weakly in $L^{p}(M, \omega)$ and the 3rd equality used the fact that $|g|$ is bounded. Thus,

$$
\begin{aligned}
F(1)-F(0) & =F\left(f_{l}\right)-F(g+\epsilon) \\
& =\int_{0}^{1} F^{\prime}(t) d t=F^{\prime}(0)+\int_{0}^{1} \int_{0}^{t} F^{\prime \prime}(s) d s d t \\
& =F^{\prime}(0)+\int_{0}^{1} \int_{0}^{t} \frac{a^{2}}{a s+b} d s d t \\
& \geq F^{\prime}(0)+\int_{0}^{1} \int_{0}^{t} \frac{a^{2}}{C} d s d t=F^{\prime}(0)+\frac{a^{2}}{2 C} .
\end{aligned}
$$

Integrating this over $M$, we have,

$$
\begin{aligned}
& \int_{M}\left(f_{l}-g-\epsilon\right)^{2} \omega^{n}=\int_{M} a^{2} \omega^{n} \\
\leq & 2 C \int_{M}(F(1)-F(0)) \omega^{n}-2 C \int_{M} F^{\prime}(0) \omega^{n} \\
= & 2 C\left(\int_{M} f_{l} \log f_{l} \omega^{n}-\int_{M}(g+\epsilon) \log (g+\epsilon) \omega^{n}\right)-2 C \int_{M} F^{\prime}(0) \omega^{n} .
\end{aligned}
$$

Using inequality (7.2), we have

$$
\begin{aligned}
& \int_{M}\left(f_{l}-g-\epsilon\right)^{2} \omega^{n} \\
\leq & C\left(\int_{M} g \log g \omega^{n}-\int_{M}(g+\epsilon) \log (g+\epsilon) \omega^{n}+o\left(\frac{1}{l}\right)\right)-C \int_{M} F^{\prime}(0) \omega^{n} \\
\leq & o\left(\epsilon+\frac{1}{l}\right)-C \int_{M} F^{\prime}(0) \omega^{n} .
\end{aligned}
$$


Consequently, we have

$$
\begin{aligned}
& 2 \lim _{l \rightarrow 0} \int_{M}\left(f_{l}-g\right)^{2} \omega^{n}=2 \lim _{\epsilon \rightarrow 0} \lim _{l \rightarrow 0} \int_{M}\left(f_{l}-g-\epsilon+\epsilon\right)^{2} \omega^{n} \\
\leq & \lim _{\epsilon \rightarrow 0} \lim _{l \rightarrow \infty} \int_{M}\left(f_{l}-g-\epsilon\right)^{2} \omega^{n}+\lim _{\epsilon \rightarrow 0} \lim _{l \rightarrow \infty} \int_{M} \epsilon^{2} \omega^{n} \\
= & \lim _{\epsilon \rightarrow 0} \lim _{l \rightarrow \infty} \int_{M}\left(f_{l}-g-\epsilon\right)^{2} \omega^{n} \\
\leq & \lim _{\epsilon \rightarrow 0} \lim _{l \rightarrow \infty} o\left(\frac{1}{l}+\epsilon\right)+C \lim _{\epsilon \rightarrow 0} \lim _{l \rightarrow \infty} \int_{M} F^{\prime}(0) \omega^{n} \\
= & 0 .
\end{aligned}
$$

Therefore, $\frac{\omega_{\phi_{l}}^{n}}{\omega^{n}}$ converges strongly to $\frac{\omega_{\phi_{0}}^{n}}{\omega^{n}}$ in $L^{2}(M, \omega)$.

\subsection{Special properties of the $\mathrm{K}$ energy minimizer}

We follow the setup in Subsection 6.2. Passing to a subsequence if necessary, there exists a $C^{1,1}$ $\operatorname{map} \underline{\phi_{0}}: \Sigma_{1} \rightarrow \mathcal{H}$ such that

1. $\phi^{(l)}$ converges to $\underline{\phi_{0}}$ weakly in $W^{2, p}\left(\Sigma^{1} \times M\right)$ for $p$ sufficiently large, with respect to a fixed Kähler metric $\pi_{2}^{*} \bar{\omega}+|d z|^{2}$.

2. $\phi^{(l)}$ converges to $\underline{\phi_{0}}$ strongly in $C^{1, \alpha}$ for any $0<\alpha<1$.

3. $\underline{\phi_{0}}(s, 0)=\phi_{0}$ and $\underline{\phi_{0}}(s, 1)=\phi_{1}$.

The first key step in this subsection is to improve weak $L^{p}(p>1)$ convergence to a strong $L^{2}$ convergence for the volume ratio $\frac{\omega_{\phi}^{n}(l)}{\omega^{n}}$.

Recalled the notation (6.7)

$$
\mathbf{E}^{(l)}(s, t)=\mathbf{E}\left(\phi^{(l)}(s, t)\right), \quad \forall(s, t) \in \Sigma_{l} .
$$

As before, $E^{(l)}$ is a uniformly bounded, weakly sub-harmonic function in $\Sigma^{(l)}$ with boundary condition (6.8).

In this subsection, we assume that both of $\phi_{0}$ and $\phi_{1}$ are Kähler metrics with constant scalar

curvature in the fixed Kähler class. Then, $\frac{\partial \mathbf{E}^{(l)}}{\partial t}=\frac{\partial \mathbf{E}^{(l)}}{\partial s}=0$ when $t=0,1$. Theorem 6.2.1 implies the following:

$$
A=B=\inf _{\phi \in \mathcal{H}} \mathbf{E}(\phi)
$$

and

$$
\mathbf{E}^{(l)}(s, t) \geq A, \quad \forall(s, t) \in \Sigma_{l}
$$


Lemma 7.2.1. As $l \rightarrow \infty$, the $L^{1}$ measure of the Laplacian $\triangle_{s, t} \mathbf{E}^{(l)}$ tends to 0 in any fixed compact subdomain.

When there is no confusion, we will drop the superscript $(l)$.

Proof. Let $\xi:(-\infty, \infty) \rightarrow \mathbb{R}$ be a smooth non-negative cut-off function such that $\xi \equiv 1$ on $\left[-\frac{1}{2}, \frac{1}{2}\right]$ and vanishes outside $\left[-\frac{3}{4}, \frac{3}{4}\right]$.

$$
\begin{aligned}
\int_{t=0}^{1} \int_{s=-\frac{l}{2}}^{\frac{l}{2}}\left|\triangle_{s, t} \mathbf{E}(s, t)\right| d s d t & \leq \int_{t=0}^{1} \int_{s=-l}^{l} \xi\left(\frac{s}{l}\right) \triangle_{s, t} \mathbf{E}(s, t) d s d t \\
& =\left.\int_{s=-l}^{l} \frac{\partial \mathbf{E}(s, t)}{\partial t}\right|_{0} ^{1} \xi\left(\frac{s}{l}\right) d s-\frac{1}{l} \int_{t=0}^{1} \int_{s=-l}^{l} \xi^{\prime}\left(\frac{s}{l}\right) \frac{\partial \mathbf{E}(s, t)}{\partial s} d s d t \\
& =0+\frac{1}{l^{2}} \int_{t=0}^{1} \int_{s=-l}^{l} \xi^{\prime \prime}\left(\frac{s}{l}\right) \mathbf{E}(s, t) d s d t \\
& \leq \frac{1}{l^{2}} \int_{t=0}^{1} \int_{s=-l}^{l}\left|\xi^{\prime \prime}\left(\frac{s}{l}\right)\right| \cdot|\mathbf{E}(s, t)| d s d t \leq \frac{1}{l^{2}} \int_{t=0}^{1} \int_{s=-l}^{l} C d s d t \\
& =\frac{C}{l} \rightarrow 0 .
\end{aligned}
$$

Lemma 7.2.2. For any point $(s, t)$ in a fixed compact domain in $\Sigma^{(l)}$, except perhaps a set of measure 0, we have $\lim _{l \rightarrow \infty} \mathbf{E}(s, t)=\lim _{l \rightarrow \infty} \mathbf{E}\left(\phi^{(l)}(s, t)\right)=A$.

Proof. Set $f^{(l)}=\triangle_{s, t} \mathbf{E}^{(l)}(s, t) \geq 0$. In $\Sigma_{\frac{l}{2}} \subset \Sigma_{l}$, we have $\lim _{l \rightarrow \infty} \int_{\Sigma_{\frac{l}{2}}} f^{(l)}=0$. Next, we decompose $\mathbf{E}^{(l)}$ into two parts:

$$
\mathbf{E}^{(l)}=u^{(l)}+v^{(l)}, \quad \text { in } \quad \Sigma_{\frac{l}{2}}
$$

such that

$$
\begin{cases}\triangle_{s, t} u^{(l)}=0, & \text { where }\left.u^{(l)}\right|_{\partial \Sigma_{\frac{l}{2}}}=E^{(l)}, \\ \triangle_{s, t} v^{(l)}=f^{(l)} \geq 0, & \text { where }\left.v^{(l)}\right|_{\partial \Sigma_{\frac{l}{2}}}=0 .\end{cases}
$$

It is clear that $v^{(l)} \leq 0$. Since $E^{(l)}$ is uniformly bounded, then $u^{(l)}$ is a bounded harmonic function in $\Sigma_{\frac{l}{2}}$ such that

$$
u^{(l)}(s, 0)=u^{(l)}(s, 1)=A, \quad \forall s \in\left[-\frac{l}{2}, \frac{l}{2}\right] .
$$

Taking limit as $l \rightarrow \infty$, in any compact subdomain $\Omega$, we have $\lim _{l \rightarrow \infty} u^{(l)}=A$. Consequently,

$$
\begin{aligned}
A & \leq \limsup _{l \rightarrow \infty} \mathbf{E}^{(l)} \\
& =\limsup _{l \rightarrow \infty}\left(u^{(l)}+v^{(l)}\right) \\
& \leq \lim _{l \rightarrow \infty} u^{(l)}=A .
\end{aligned}
$$


Therefore, for every point in $\Omega$ (fixed), we have

$$
\lim _{l \rightarrow \infty} \mathbf{E}^{(l)}=\limsup _{l \rightarrow \infty} \mathbf{E}^{(l)}=A .
$$

Combining this with Theorem 7.1.1, we have

Corollary 7.2.3. For any $(s, t) \in \Sigma_{1}$, except perhaps a set of measure 0 in $\Sigma_{1}$, the volume ratio $\frac{\omega_{\phi}^{n}(l)}{\omega^{n}}$ converges strongly in $L^{2}(M, \omega)$ sense.

This corollary is crucial in the following arguments.

For notational simplicity, set $\phi_{l}=\phi^{(l)} \in \mathcal{H}$. Let $\zeta(s)=\frac{1}{1+s^{2}}$. Then

$$
\int_{z \in \Sigma_{l}} \int_{M} \omega^{n} \zeta(z) d z \wedge d \bar{z} \leq C, \forall l \in(1, \infty)
$$

Lemma 7.2.1 and Theorem 6.1.1 imply

$$
\int_{\Sigma_{\frac{l}{2}}} \int_{M}\left|\frac{\partial \eta^{l, \alpha}}{\partial w_{\bar{\beta}}}\right|_{\phi_{l}}^{2} \omega_{\phi_{l}}^{n} d z \wedge d \bar{z} \leq \int_{z \in \Sigma_{\frac{l}{2}}} \triangle_{z} E(z, \cdot) d z \wedge d \bar{z} \rightarrow 0
$$

where

$$
\eta^{l, \alpha}=-g_{\phi_{l}}^{\alpha \bar{\beta}} \frac{\partial^{2} \phi_{l}}{\partial z \partial w_{\bar{\beta}}}, \quad \text { and } \quad g_{\phi_{l}, \alpha \bar{\beta}}=g_{\alpha \bar{\beta}}+\frac{\partial^{2} \phi_{l}}{\partial w_{\alpha} \partial w_{\bar{\beta}}} .
$$

When there is no confusion arised, we will drop the dependence on $l$.

Now we adopte a sympletic point of view now: For any $l>1$, the product manifold $\Sigma_{l} \times M$ is foliated by smooth holomorphic discs which are transversal to $M$ direction, dictated by the structure of almost smooth solutions $\phi_{l}$ of HCMA equation 1.1. In other words, for any $z_{0}=$ $t_{0}+\sqrt{-1} s_{0} \in \Sigma_{1}$ fixed, we may use $\left\{z_{0}\right\} \times M$ as the parametrization space of the set of holomorphic discs which are transversal to $\left\{z_{0}\right\} \times M$. Note that this parametrization is effective except a set of codimension 2. Along each holomphic disc, the $(n, n)$ form $\omega_{\phi}^{n}$ is invariant. The above two inequalities can be re-stated as:

$$
\int_{M} \int_{\Sigma_{l}} \frac{\omega^{n}}{\omega_{\phi_{l}}^{n}} \zeta(z) d z \wedge d \bar{z} \wedge \omega_{\phi_{l}}^{n} \leq C
$$

and

$$
\int_{M} \int_{\Sigma_{\frac{l}{2}}}\left|\frac{\partial \eta^{l, \alpha}}{\partial w_{\bar{\beta}}}\right|_{\phi_{l}}^{2} d z \wedge d \bar{z} \wedge \omega_{\phi_{l}}^{n} \rightarrow 0
$$


Choosing any point $z_{0}$ in the interior of $\Sigma_{1}$ such that $\frac{\omega_{\phi_{l}}^{n}\left(z_{0}, \cdot\right)}{\omega^{n}}$ converges strongly to $\frac{\omega_{\Phi_{0}}^{n}\left(z_{0}, \cdot\right)}{\omega^{n}}$ in $L^{2}(M, \omega)$. For any $L^{2}$ function $h$ in $M$, we can normalize it by the following

$$
h(x)=\left\{\begin{array}{lc}
\lim _{\epsilon \rightarrow 0} \oint_{B_{\epsilon}(x)} h, & \text { if limit exists } \\
0, & \text { otherwise }
\end{array}\right.
$$

Then, $h(x)$ differs from the original function at most at a set of measure 0 . Now, we decompose $\left\{z_{0}\right\} \times M$ into the union of two subsets $F_{1}$ and $F_{2}$ such that

$$
\frac{\omega_{\phi_{0}}{ }^{n}}{\omega^{n}}\left(z_{0}, x\right)>0, \forall x \in F_{1}
$$

and

$$
\frac{\omega_{\phi_{0}}^{n}}{\omega^{n}}\left(z_{0}, x\right)=0, \forall x \in F_{2} \text {. }
$$

Clearly, $\operatorname{mes}\left(F_{1}\right)>0$ since

$$
\begin{aligned}
\operatorname{mes}\left(F_{1}\right) & =\int_{F_{1}} \omega^{n} \\
& \geq c \int_{F_{1}} \frac{\omega_{\phi_{0}}{ }^{n}}{\omega^{n}{ }^{n}}\left(z_{0}, x\right) \omega^{n} \\
& =c \int_{M} \frac{\omega_{\phi_{0}}}{\omega^{n}}\left(z_{0}, x\right) \omega^{n}=\operatorname{cvol}(M)>0,
\end{aligned}
$$

where $\frac{1}{c}$ is the upper bound of the volume form ratio $\frac{\omega_{\phi_{0}}{ }^{n}}{\omega^{n}}\left(z_{0}, \cdot\right)$ in $M$.

Ultimately, we want to show that mes $\left(F_{2}\right)=0$. This is not attainable at this point. However, we can prove the following strong statement about the volume form ratio in the limit.

Theorem 7.2.4. There exists a uniform constant $\epsilon_{0}$, which depends only on $\varphi_{0}, \varphi_{1} \in \mathcal{H}$ (in particular, independent of $x)$, such that, excluding at most a set of measure 0 from $F_{1}$, we have

$$
\frac{\omega_{\phi_{0}}{ }^{n}}{\omega^{n}}\left(z_{0}, x\right)>\epsilon_{0}, \quad \forall x \in F_{1} .
$$

Proof. By our choice of $z_{0}, \frac{\omega_{\phi_{l}}{ }^{n}}{\omega^{n}}\left(z_{0}, x\right) \rightarrow \frac{\omega_{\phi_{0}}{ }^{n}}{\omega^{n}}\left(z_{0}, x\right)$ strongly in $L^{2}(M, \omega)$. For any $\delta>0$, there exists an open set $E_{\delta}$ with measure $E_{\delta}<\frac{\delta}{2}$ such that $\frac{\omega_{\phi_{l}}{ }^{n}}{\omega^{n}}\left(z_{0}, x\right) \rightarrow \frac{\omega_{\phi_{0}}{ }^{n}}{\omega^{n}}\left(z_{0}, x\right)$ pointwisely in $\left\{z_{0}\right\} \times\left(M \backslash E_{\delta}\right)$. Set $\ell_{l}(z):\left\{z_{o}\right\} \times M \rightarrow\{z\} \times M$ as ths syndromy map such that $\left(z_{0}, x\right)$ and $\left(z, \ell_{l}(z)(x)\right)$ lies in the same holomorphic disc. Then, $\ell_{l}(z)$ is well defined for generic point of $x \in M$. Now set

$$
S_{l}(z, x)=\left|\frac{\partial \eta^{l, \alpha}}{\partial w_{\bar{\beta}}}\right|_{\phi_{l}}^{2}\left(z, \ell_{l}(z)(x)\right) \quad \text { and } \quad f_{l}(z, x)=\frac{\omega^{n}}{\omega_{\phi_{l}}^{n}}\left(z, \ell_{l}(z)(x)\right) \zeta(z) .
$$


Denote

$$
S_{l}(x)=\int_{\Sigma_{\frac{l}{2}}} S_{l}(z, x) d z \wedge d \bar{z} \quad \text { and } \quad f_{l}(x)=\int_{\Omega} f_{l}(z, x) d z \wedge d \bar{z} .
$$

Then, equations (7.5) and (7.6) imply

$$
\int_{M} S_{l}(x) \cdot \frac{\omega_{\phi_{l}}^{n}}{\omega^{n}} \omega^{n} \rightarrow 0, \quad \text { and } \quad \int_{M} f_{l}(x) \cdot \frac{\omega_{\phi_{l}}^{n}}{\omega^{n}} \omega^{n} \leq C .
$$

The first assertion implies that $\sqrt{S_{l}(x)} \cdot \sqrt{\frac{\omega_{\phi_{l}}^{n}}{\omega^{n}}} \rightarrow 0$ strongly in $L^{2}(M)$. Consequently, $\left\{S_{l}(x)\right\}$ uniformly converges to 0 in $\left(M \backslash E_{\delta}\right) \cap F_{1}$. On the other hand, there exists a set $E_{\delta}^{\prime}$ of measure at most $\frac{\delta}{2}$ such that

$$
\liminf _{l \rightarrow \infty}\left(f_{l}(x) \cdot \frac{\omega_{\phi_{l}}^{n}}{\omega^{n}}\right)<C(\delta), \quad \text { whenever } x \in M \backslash E_{\delta}^{\prime}
$$

Let $F_{\delta}=F \subset\left(E_{\delta} \bigcup E_{\delta}^{\prime}\right)$. Then

$$
\operatorname{mes}\left(F_{\delta}\right) \geq \operatorname{mes}(F)-\delta \text {. }
$$

We proceed to prove that our theorem holds in $F_{\delta}$.

Let us pick any point $x_{0} \in F_{\delta}$ and fix it for now. Passing to a subsequence if necessary, we may assume (w.l.o.g.) that

$$
\frac{\omega_{\phi_{l}}^{n}}{\omega^{n}}\left(z_{0}, x_{0}\right)=\frac{\omega_{\phi_{0}}^{n}}{\omega^{n}}\left(z_{0}, x_{0}\right)=\epsilon>0
$$

Here $\epsilon>0$ may be very small. The goal is to show that a uniform positive lower bound of the volume form ratio exists. Clearly, $S_{l}\left(x_{0}\right) \rightarrow 0$ for any fixed compact subset $^{25} \Omega$. Next,

$$
\liminf _{l \rightarrow \infty}\left(f_{l}\left(x_{0}\right) \cdot \frac{\omega_{\phi_{l}}^{n}}{\omega^{n}}\left(z_{0}, x_{0}\right)\right)<C(\delta) .
$$

Passing to a subsequence if necessary, we have

$$
f_{l}\left(x_{0}\right) \leq C\left(\delta, \epsilon, x_{0}\right),
$$

in this subsequence. The main point is that it has a uniform bound in terms of this subsequence.

\footnotetext{
${ }^{25}$ As a matter of fact, we may choose $\Omega=\left[-\frac{l}{2}, \frac{l}{2}\right]$ and our claim still holds.
} 
For any $l$, consider any holomorphic disc which passes through $\left(z_{0}, x_{0}\right)$ and denote it by:

$$
\ell_{l}:\left(\Sigma_{(l)}, \partial \Sigma\right) \rightarrow\left(\Sigma_{(l)} \times M,\left(\partial \Sigma_{(l)}\right) \times M\right)
$$

such that $l\left(z_{0}\right)=x_{0}$. It is easy to see that this holomorphic disc has uniformly bounded area in any fixed compact sub-domain (c.f. Section 4). Let

$$
g_{l}(z)=\log \frac{\omega_{\phi_{l}}^{n}}{\omega^{n}}\left(z, \ell_{l}(z)\right), \quad \forall z \in \Sigma_{(l)} .
$$

By definition, $g_{l}$ is uniformly bounded in the boundary $\partial \Sigma_{l}$. Then

$$
g_{l}\left(z_{0}\right)=\log \frac{\omega_{\phi_{l}}^{n}}{\omega^{n}}\left(z_{0}, \ell_{l}\left(z_{0}\right)\right)=\log \frac{\omega_{\phi_{l}}^{n}}{\omega^{n}}\left(z_{0}, x_{0}\right)=\ln \epsilon, \quad \forall l .
$$

Moreover, there exists a uniform constant $C$ such that $\left|g_{l}(z)\right| \leq C$ in the boundary $\partial \Sigma_{(l)}$. Since $\ell_{l}(\Sigma)$ is a measure 0 set in $\Sigma \times M$, the limit of $g_{l}(z)(z \neq 0)$ most likely have no bearing on $\log \frac{\omega_{\phi_{0}}^{n}}{\omega^{n}}$ in $\Sigma \times M$. However, we are interested in obtaining a uniform positive lower bound on $\epsilon$ through this procedure.

Recall that Corollary 4.9 implies that

$$
\triangle_{z} g_{l}(z)=S\left(z, \ell_{l}(z)\right)+\left.R_{0, \alpha \bar{\beta}} \eta^{l, \alpha} \eta^{l, \bar{\beta}}\right|_{\left(z, \ell_{l}(z)\right)} .
$$

Split $g_{l}=u_{l}+v_{l}$ such that

$$
\triangle_{z} v_{l}=\left.R_{0, \alpha \bar{\beta}} \eta^{l, \alpha} \eta^{l, \bar{\beta}}\right|_{\left(z, \ell_{l}(z)\right)}
$$

and

$$
v_{l}(z)=g_{l}(z), \quad \forall z \in \partial \Sigma .
$$

Claim: There exists a uniform constant $C$ such that $\left|v_{l}\right|<C$.

Recall that Corollary 4.2 .4 implies that

$$
-\triangle_{z} \phi_{l}\left(z, \ell_{l}(z)\right)=g_{0, \alpha \bar{\beta}} \eta^{l, \alpha} \eta^{l, \bar{\beta}}, \forall z \in \Sigma_{l} .
$$

Note that $\phi_{l}\left(z, \ell_{l}(z)\right)$ has a uniform bound in $\Sigma_{l}$. There exists a constant $C$ such that

$$
-C g_{0, \alpha \bar{\beta}}<R_{0, \alpha \bar{\beta}}<C g_{0, \alpha \bar{\beta}}
$$

Consequently,

$$
\triangle_{z}\left(v_{l}-C \phi_{l}\right)<0<\triangle_{z}\left(v_{l}+C \phi_{l}\right) .
$$

By maximum principle, we have $\left|v_{l}\right| \leq C$ and our earlier claim holds. Next,

$$
\triangle_{z} \quad u_{l}=S_{l}(z, \ell(z))>0,
$$


and $\left.u_{l}\right|_{\partial \Sigma_{l}}=0$. Obviously $u_{l} \leq 0$ (maximum principle). Moreover

$$
\begin{aligned}
\int_{\Sigma_{l}} e^{-u_{l}}(z) \cdot \zeta(z) d z \wedge d \bar{z} & \leq C \int_{\Sigma_{l}} e^{-\log \frac{\omega_{\phi_{l}}^{n}}{\omega^{n}}}(z) \cdot \zeta(z) d z \wedge d \bar{z} \\
& =\int_{\Sigma_{l}}\left(\frac{\omega^{n}}{\omega_{\phi_{l}}^{n}}\right)\left(z, \ell_{l}(z)\right) \cdot \zeta(z) d z \wedge d \bar{z} \\
& =\int_{\Sigma_{l}} f_{l}\left(z, \ell_{l}(z)\right) \cdot \zeta(z) d z \wedge d \bar{z} \\
& =f_{l}\left(x_{0}\right) \leq C\left(z_{0}, \delta, \epsilon\right),
\end{aligned}
$$

For any small positive number $\delta_{1}>0$, Theorem 4.2.13 implies

$$
0 \leq \triangle_{z} u_{l}=S\left(z, \ell_{l}(z)\right)<\frac{C}{\delta_{1}^{2}}, \quad \forall z \in\left[-l+\delta_{1}, l-\delta_{1}\right] \times\left[\delta_{1}, 1-\delta_{1}\right]
$$

These two conditions imply that $u_{l}$ converges strongly in $W^{1, \alpha}$ in any fixed compact sub-domain of $\left(-l+\delta_{1}, l-\delta_{1}\right) \times\left(\delta_{1}, 1-\delta_{1}\right)$. Moreover,

$$
\int_{\Sigma_{\frac{l}{2}}}\left|\triangle_{z} u_{l}\right|=\int_{\Sigma_{\frac{l}{2}}} S(z, \ell(z))=S_{l}\left(x_{0}\right) \rightarrow 0 .
$$

Passing to a subsequence if necessary, there exists a non-negative harmonic function $u_{\infty}$ in $(-\infty, \infty) \times[0,1]$ such that for any fixed compact subset $\Omega$, we have

$$
u_{l} \rightarrow u_{\infty}
$$

weakly in $L^{p}(\Omega)$ for any $p>1$; and it converges strongly to $u_{\infty}$ in $C^{1, \alpha}(0<\alpha<1)$ in any fixed compact sub-domain. Moreover, $u_{\infty}=0$ in the boundary of this infinite long strip $\Sigma_{\infty}=$ $(-\infty, \infty) \times[0,1]$ and

$$
\int_{\Sigma_{\infty}} e^{-u_{l}}(z) \cdot \zeta(z) d z \wedge d \bar{z}<C\left(\delta, \epsilon, x_{0}\right) .
$$

The only harmonic function with this growth condition in $\Sigma_{\infty}$ is a constant function. Thus, $u_{\infty} \equiv 0$. In particular, for any fixed sub-domain $\Omega \subset\left(-l+\delta_{1}, l-\delta_{1}\right) \times\left(\delta_{1}, 1-\delta_{1}\right)$, we have $u_{l} \rightarrow 0$ strongly. In particular,

$$
\lim _{l \rightarrow \infty} u_{l}\left(z_{0}\right)=0
$$

It follows,

$$
\begin{aligned}
\log \epsilon & =g_{l}\left(z_{0}\right) \\
& =u_{l}\left(z_{0}\right)+v_{l}\left(z_{0}\right)>-C .
\end{aligned}
$$

Consequently, we have

$$
\frac{\omega_{\phi_{0}}^{n}}{\omega^{n}}\left(z_{0}, x_{0}\right)>e^{-C}=\epsilon_{0} .
$$

Note that we chose $x_{0}$ arbitrarily in $F_{\delta}$, thus the lower bound $e^{-C}=\epsilon_{0}$ holds for every point in $F_{\delta}$. Since $\operatorname{mes}\left(F \backslash F_{\delta}\right)<\delta$, our theorem is then proved. 


\subsection{A regularity theorem for a $C^{1,1}$ minimizer of the $\mathrm{K}$ energy functional and the weak "Kähler Ricci" flow}

In this subsection, we want to prove a regularity lemma for any $C^{1,1}$ minimizer of the $\mathrm{K}$ energy in an arbitrary Kähler class.

Theorem 7.3.1. Suppose $\underline{\phi_{0}} \in C^{1,1}(M)$ is in the closure of $\mathcal{H}$ under weak $C^{1,1}$ topology. If the $K$ energy functional has a uniform lower bound in this Kähler class and $E\left(\underline{\phi_{0}}\right)$ realizes the infimum of the K energy functional in this Kähler class, then $\left(\frac{\omega_{\phi_{0}}^{n}}{\omega^{n}}\right)^{\frac{1}{2}}$ is in $W^{1,2}(M, \omega)$.

We want to prove this via the "Kähler-Ricci" flow ${ }^{26}$.

Proof. Let $\phi_{0}(s)(0 \leq s \leq 1)$ be a 1-parameter Kähler potentials such the following holds:

1. $\phi_{0}(0)=\underline{\phi_{0}}$ and $\phi_{0}(s)(0<s \leq 1) \in \mathcal{H}$.

2. $\phi_{0}(s)$ has uniform $C^{1,1}$ upper bound and $\phi_{0}(s)(s>0) \rightarrow \underline{\phi_{0}}$ strongly in $W^{2, p}(M, \omega)$ for $p$ large enough.

Applying the "Kähler-Ricci flow" to this 1-parameter family of Kähler potentials $\phi_{0}(s)(0<s \leq$ 1) :

$$
\begin{aligned}
\frac{\partial \phi(s, t)}{\partial t} & =\log \frac{\omega_{\phi}^{n}}{\omega^{n}} \\
\phi(s, 0) & =\phi_{0}(s) .
\end{aligned}
$$

Clearly, for each $s>0$ fixed, there exists a uniform $C^{2, \alpha}$ bound for $\phi(s, t)(s>0,0 \leq t \leq \infty)$. However, the upper-bound may depends on $s$ and in particular, it may blows up when $t, s$ are both approaching 0 .

Claim 1: There exists a uniform constant $C$ which is independent of the parameters $s$ and $t$ such that

$$
n+\triangle_{0} \phi \leq C, \quad \forall(s, t) \in(0,1] \times[0, \infty) .
$$

Here we used $\triangle_{\phi}, \triangle_{0}$ to denote the Lapalacian operators of the Kähler metrics $\omega_{\phi}, \omega$ respectively.

During this proof, we use $C$ to denote a generic constant which is independent of $s, t$. Taking the derivatives of the flow equation (7.7), we obtain

$$
\frac{\partial^{2} \phi}{\partial t^{2}}=\triangle_{\phi}\left(\frac{\partial \phi(s, t)}{\partial t}\right)
$$

\footnotetext{
${ }^{26}$ This is not Kähler Ricci flow in the usual sense since we are not in the Canonical class and the first Chern class might not have a sign.
} 
This implies that

$$
\begin{aligned}
\frac{\omega_{\phi(s, t)}^{n}}{\omega^{n}} & =e^{\frac{\partial \phi(s, t)}{\partial t}} \leq \max _{x \in M} e^{\frac{\partial \phi(s, t, x)}{\partial t}} \\
& \leq\left.\max _{x \in M} e^{\frac{\partial \phi(s, t, x)}{\partial t}}\right|_{t=0}=\max _{x \in M} \frac{\omega_{\phi(s, 0, x)}^{n}}{\omega^{n}} \\
& =\max _{x \in M} \frac{\omega_{\phi_{0}(s, x)}^{n}}{\omega^{n}} \leq C .
\end{aligned}
$$

In other words, we have a uniform upper-bound on the evolved volume form.

Following the calculation in 33], it is straightforward to show (for each fixed $s>0$ )

$$
\begin{aligned}
& \left(\triangle_{\phi}-\frac{\partial}{\partial t}\right)\left(\exp (-\lambda \phi)\left(n+\triangle_{0} \phi\right)\right) \\
& \geq \quad-\exp (-\lambda \phi)\left(n^{2} \inf _{i \neq 1}\left(R_{\bar{i} \bar{i} \overline{1}}\right)\right)-\lambda \exp (-\lambda \phi)\left(n-\log \frac{\omega_{\phi}^{n}}{\omega^{n}}\right)\left(n+\triangle_{0} \phi\right) \\
& \quad+\left(\lambda+\inf _{i \neq 1}\left(R_{i \bar{i} \overline{1} \overline{1}}\right) \exp (-\lambda \phi) \cdot\left(\frac{\omega^{n}}{\omega_{\phi}^{n}}\right)^{\frac{1}{n}}(n+\triangle \phi)^{\frac{n}{n-1}},\right.
\end{aligned}
$$

where $R_{i \bar{i} \overline{1}}$ is the bisectional curvature of the Kähler metric (corresponds to $\omega$ ) and $\lambda$ is a positive number such that

$$
\lambda+\inf _{i \neq 1} R_{i \bar{i} 1 \overline{1}}>1
$$

Multiplying $\left(\frac{\omega_{\phi}^{n}}{\omega^{n}}\right)^{\frac{1}{n}}$ on both sides, we get

$$
\begin{aligned}
&\left(\frac{\omega_{\phi}^{n}}{\omega^{n}}\right)^{\frac{1}{n}}\left(\triangle_{\phi}-\frac{\partial}{\partial t}\right)\left(\exp (-\lambda \phi)\left(n+\triangle_{0} \phi\right)\right) \\
& \geq-\exp (-\lambda u)\left(n^{2} \inf _{i \neq 1}\left(R_{\bar{i} \overline{1} 1 \overline{1}}\right)\right)\left(\frac{\omega_{\phi}^{n}}{\omega^{n}}\right)^{\frac{1}{n}}-\lambda \exp (-\lambda \phi)\left(n-\log \frac{\omega_{\phi}^{n}}{\omega^{n}}\right)\left(\frac{\omega_{\phi}^{n}}{\omega^{n}}\right)^{\frac{1}{n}}\left(n+\triangle_{0} \phi\right) \\
& \quad+\left(C_{0}+\inf _{i \neq 1}\left(R_{i \bar{i} 1 \overline{1}}\right)\right) \exp \left(-C_{0} \phi\right) \cdot(n+\triangle \phi)^{\frac{n}{n-1}},
\end{aligned}
$$

If $\phi$ is uniformly bound (independent of $s, t)$, then (recall that $\left(\frac{\omega_{\phi}^{n}}{\omega^{n}}\right)^{\frac{1}{n}}<C$ )

$$
\left(\frac{\omega_{\phi}^{n}}{\omega^{n}}\right)^{\frac{1}{n}}\left(\triangle_{\phi}-\frac{\partial}{\partial t}\right) v \geq-c_{1}-c_{2} v+c_{0} v^{\frac{n}{n-1}} .
$$

where $c_{0}, c_{1}, c_{2}$ are uniform positive constants and $v=\exp (-\lambda \phi)\left(n+\triangle_{0} \phi\right)$. Therefore,

$$
v(s, t) \leq v(s, 0) \leq C .
$$


In other words, there is a uniform constant $C$ such that

$$
0 \leq n+\triangle_{0} \phi(s, t) \leq C,
$$

provided that $\phi(s, t)$ has a uniform $C^{0}$ bound. It is easy to see that we have uniform upper bound on $\frac{\partial \phi}{\partial t}$. On the other hand, at minimum point of $\phi$, we have

$$
\log \frac{\omega_{\phi}^{n}}{\omega^{n}} \geq 0
$$

Thus, in the barrier sense, we have

$$
\frac{\partial \min _{M} \phi}{\partial t} \geq 0
$$

Consequently,

$$
|\phi(s, t)| \leq C .
$$

This concludes the proof of our first claim above.

For any sequence of number $s_{i}, t_{i} \rightarrow 0$, set

$$
\phi_{i}=\phi\left(s_{i}, t_{i}\right), \quad \text { and } \quad \phi_{0 i}=\phi\left(s_{i}, 0\right)=\phi_{0}\left(s_{i}, 0\right) .
$$

Passing to a subsequence if necessary, we have that $\phi_{i}$ converges to some $C^{1,1}$ Kähler potential $\tilde{\phi}_{0}$ (strongly in $C^{1, \alpha}(\forall \alpha<1)$ and weakly in $W^{2, p}$ ( $p$ large enough)). Note that $\tilde{\phi}_{0}$ does not necessary equal to $\phi_{0}$ even though $t_{i}, s_{i} \rightarrow 0$ !

Claim 2: $\omega_{\tilde{\phi}_{0}}^{n} \equiv \omega_{\phi_{0}}^{n}$ and $\frac{\omega_{\phi_{i}}^{n}}{\omega^{n}}$ converge strongly to $\frac{\omega_{\phi_{0}}^{n}}{\omega^{n}}$ in $L^{2}(M, \omega)$.

To prove this claim, choose an arbitrary smooth non-negative cut off function $\chi$ (fixed) and

$$
\begin{aligned}
\frac{1}{2} \frac{d}{d t} \int_{M} \chi\left(\frac{\omega_{\phi}^{n}}{\omega^{n}}\right)^{2} \omega^{n} & =\int_{M} \chi \triangle_{\phi} \log \frac{\omega_{\phi}^{n}}{\omega^{n}} \cdot\left(\frac{\omega_{\phi}^{n}}{\omega^{n}}\right) \omega_{\phi}^{n} \\
& =\int_{M} \chi\left(\triangle_{\phi} \frac{\omega_{\phi}^{n}}{\omega^{n}}-\left|\nabla \log \frac{\omega_{\phi}^{n}}{\omega^{n}}\right|_{\phi}^{2} \frac{\omega^{n}}{\omega_{\phi}^{n}}\right) \omega_{\phi}^{n} \\
& \leq \int_{M} \triangle_{\phi} \chi\left(\frac{\omega_{\phi}^{n}}{\omega^{n}}\right) \omega_{\phi}^{n} \leq C .
\end{aligned}
$$

The last inequality holds since the evolving Kähler potentials have a uniform $C^{1,1}$ upperbound and $\chi$ is a fixed smooth function. Integrating this inequality from $t=0$ to $t=t_{i}$, we have

$$
\begin{aligned}
\left.\int_{M} \chi\left(\frac{\omega_{\phi_{i}}^{n}}{\omega^{n}}\right)^{2} \omega^{n}\right|_{s_{i}, t_{i}} & =\left.\int_{M} \chi\left(\frac{\omega_{\phi_{0}}^{n}}{\omega^{n}}\right)^{2} \omega^{n}\right|_{s_{i}, 0}+\int_{0}^{t_{i}} \frac{d}{d t} \int_{M} \chi\left(\frac{\omega_{\phi}^{n}}{\omega^{n}}\right)^{2} \omega^{n} d t \\
& \leq \int_{M} \chi\left(\frac{\omega_{\phi_{0}\left(s_{i}\right)}}{\omega^{n}}\right)^{2} \omega^{n}+C t_{i} .
\end{aligned}
$$


On the other hand, $\frac{\omega_{\phi_{i}}^{n}}{\omega^{n}}$ converges weakly to $\frac{\omega_{\tilde{\phi}_{0}}^{n}}{\omega^{n}}$ in $L^{2}(M, \omega)$. Then

$$
\begin{aligned}
\int_{M} \chi\left(\frac{\omega_{\tilde{\phi}_{0}}^{n}}{\omega^{n}}\right)^{2} \omega^{n} & \leq \lim _{i \rightarrow \infty} \int_{M} \chi\left(\frac{\omega_{\phi_{i}}^{n}}{\omega^{n}}\right)^{2} \omega^{n} \\
& \leq \lim _{i \rightarrow \infty}\left(\int_{M} \chi\left(\frac{\omega_{\phi_{0}}^{n}}{\omega^{n}}\right)^{2} \omega^{n}+C t_{i}\right) \\
& =\int_{M} \chi\left(\frac{\omega_{\phi_{0}}^{n}}{\omega^{n}}\right)^{2} \omega^{n}
\end{aligned}
$$

The last equality holds since $\phi_{0 i}$ converges strongly to $\phi_{0}$ in $W^{2, p}(M, \omega)$ for $p$ large enough (by our assumption in the beginning). This holds for any non-negative smooth cut off function in $M$. Consequently, we have

$$
0 \leq \frac{\omega_{\tilde{\phi_{0}}}^{n}}{\omega^{n}} \leq \frac{\omega_{\phi_{0}}^{n}}{\omega^{n}}
$$

a.e. in $M$. However,

$$
\int_{M} \frac{\omega_{\tilde{\phi_{0}}}^{n}}{\omega^{n}} \omega^{n}=\int_{M} \frac{\omega_{\phi_{0}}^{n}}{\omega^{n}} \omega^{n}=\operatorname{vol}(M) !
$$

Consequently,

$$
\omega_{\tilde{\phi_{0}}}^{n} \equiv \omega_{\phi_{0}}^{n}
$$

in the sense of $L^{2}(M, \omega)$. The uniqueness of $C^{1,1}$ solution to the Monge-Ampere equation implies that $\tilde{\phi}_{0}=\phi_{0}$. In particular, this implies the K energy $E\left(\phi_{i}\right)$ converges to $\mathbf{E}\left(\phi_{0}\right)$.

On the other hand, the equality (7.12) forces equality in (7.9)-(7.11) to hold. In particular, we have

$$
\int_{M} \chi\left(\frac{\omega_{\tilde{\phi_{0}}}^{n}}{\omega^{n}}\right)^{2} \omega^{n}=\lim _{i \rightarrow \infty} \int_{M} \chi\left(\frac{\omega_{\phi_{i}}^{n}}{\omega^{n}}\right)^{2} \omega^{n} .
$$

Thus, $\frac{\omega_{\phi_{i}}^{n}}{\omega^{n}}$ converges strongly to $\frac{\omega_{\tilde{\phi}_{0}}^{n}}{\omega^{n}}$ in $L^{2}(M, \omega)$. Our second claim is then proved.

Now we used these two claims to prove our theorem. Consider $\mathbf{E}(s, t)=\mathbf{E}(\phi(s, t))(0<s \leq$ 1 and $0 \leq t<\infty)$. Set

$$
A=\inf _{\phi \in \mathcal{H}} \mathbf{E}(\phi)>-\infty .
$$

By our assumption on $\phi_{0}$, we have

$$
\mathbf{E}\left(\phi_{0}\right)=A=\lim _{s \rightarrow 0} \mathbf{E}(\phi(s, 0)) \leq \mathbf{E}(s, t), \quad \forall s>0, t \geq 0 .
$$


For any fixed number $c_{0}>0$, it is straightforward to show that there exists a sequence $s_{i}, t_{i} \rightarrow 0$ such that

$$
\begin{aligned}
-c_{0} & \leq\left.\frac{\partial \mathbf{E}(s, t)}{\partial t}\right|_{s_{i}, t_{i}} \\
& =\left.\int_{M} \log \frac{\omega_{\phi}^{n}}{\omega^{n}} \triangle_{\phi} \frac{\partial \phi}{\partial t} \omega_{\phi}^{n}\right|_{s_{i}, t_{i}}-\left.\int_{M} \frac{\partial \phi}{\partial t}\left(\operatorname{Ric}\left(\omega_{0}\right)-\underline{R} \omega_{\phi}\right) \wedge \omega_{\phi}^{n-1}\right|_{s_{i}, t_{i}} \\
& =-\left.\int_{M}\left|\nabla \log \frac{\omega_{\phi}^{n}}{\omega^{n}}\right|_{\phi}^{2} \omega_{\phi}^{n}\right|_{s_{i}, t_{i}}-\left.\int_{M}\left(\log \frac{\omega_{\phi}^{n}}{\omega^{n}}-C\right)\left(\operatorname{Ric}\left(\omega_{0}\right)-\underline{R} \omega_{\phi}\right) \wedge \omega_{\phi}^{n-1}\right|_{s_{i}, t_{i}} \\
& =-\left.\int_{M}\left|\nabla \log \frac{\omega_{\phi}^{n}}{\omega^{n}}\right|_{\phi}^{2} \omega_{\phi}^{n}\right|_{s_{i}, t_{i}}-\left.c \int_{M}\left(\log \frac{\omega_{\phi}^{n}}{\omega^{n}}-C\right) \omega \wedge \omega_{\phi}^{n-1}\right|_{s_{i}, t_{i}} .
\end{aligned}
$$

Here $c, C$ are some uniform positive number such that

$$
\log \frac{\omega_{\phi}^{n}}{\omega^{n}}<C+1
$$

and

$$
\operatorname{Ric}\left(\omega_{0}\right)-\underline{R} \omega_{\phi} \leq c \omega .
$$

Thus

$$
\begin{aligned}
\int_{M}\left|\nabla \log \frac{\omega_{\phi_{i}}^{n}}{\omega^{n}}\right|_{\phi_{i}}^{2} \omega_{\phi_{i}}^{n} & \leq c_{0}-c \int_{M}\left(\log \frac{\omega_{\phi_{i}}^{n}}{\omega^{n}}-C\right)\left(\omega+\sqrt{-1} \partial \bar{\partial} \phi_{i}-\sqrt{-1} \partial \bar{\partial} \phi_{i}\right) \wedge \omega_{\phi_{i}}^{n-1} \\
& \leq c_{0}+c \int_{M}\left(\log \frac{\omega_{\phi_{i}}^{n}}{\omega^{n}}-C\right) \sqrt{-1} \partial \bar{\partial} \phi_{i} \wedge \omega_{\phi_{i}}^{n-1}-c \int_{M}\left(\log \frac{\omega_{\phi_{i}}^{n}}{\omega^{n}}-C\right) \omega_{\phi_{i}}^{n} \\
& \leq c_{0}-c \int_{M} \sqrt{-1} \partial \log \frac{\omega_{\phi_{i}}^{n}}{\omega^{n}} \wedge \bar{\partial} \phi_{i} \wedge \omega_{\phi_{i}}^{n-1}-c \int_{M}\left(\log \frac{\omega_{\phi_{i}}^{n}}{\omega^{n}}-C\right) \frac{\omega_{\phi_{i}}^{n}}{\omega^{n}} \omega^{n} \\
& \leq c_{0}+c\left(\epsilon \int_{M}\left|\nabla \log \frac{\omega_{\phi_{i}}^{n}}{\omega^{n}}\right|_{\phi_{i}}^{2} \omega_{\phi_{i}}^{n}+\frac{1}{\epsilon} \int_{M} \sqrt{-1} \partial \phi_{i} \wedge \bar{\partial} \phi_{i} \wedge \omega_{\phi_{i}}^{n-1}\right)+C \\
& \leq C(\epsilon)+c \epsilon \int_{M}\left|\nabla \log \frac{\omega_{\phi_{i}}^{n}}{\omega^{n}}\right|_{\phi_{i}}^{2} \omega_{\phi_{i}}^{n} .
\end{aligned}
$$

Choose $\epsilon$ small enough so that $c \epsilon<\frac{1}{2}$. With this $\epsilon$, we have

$$
\begin{aligned}
\int_{M}\left|\nabla \sqrt{\frac{\omega_{\phi_{i}}^{n}}{\omega^{n}}}\right|_{\omega}^{2} \omega^{n} & \leq C \int_{M}\left|\nabla \sqrt{\frac{\omega_{\phi_{i}}^{n}}{\omega^{n}}}\right|_{\phi_{i}}^{2} \omega^{n} \\
& =C \int_{M}\left|\nabla \log \frac{\omega_{\phi_{i}}^{n}}{\omega^{n}}\right|_{\phi_{i}}^{2} \omega_{\phi_{i}}^{n} \leq C .
\end{aligned}
$$

Letting $i \rightarrow \infty$, we see that

$$
\int_{M}\left|\nabla \sqrt{\frac{\omega_{\tilde{\tilde{\phi}_{0}}}^{n}}{\omega^{n}}}\right|_{\omega}^{2} \omega^{n} \leq C .
$$

Since $\frac{\omega_{\phi_{0}}^{n}}{\omega^{n}} \equiv \frac{\omega_{\phi_{0}}^{n}}{\omega^{n}}$, we have

$$
\int_{M}\left|\nabla \sqrt{\frac{\omega_{\phi_{0}}^{n}}{\omega^{n}}}\right|_{\omega}^{2} \omega^{n} \leq C .
$$

The theorem is then proved. 


\section{The Problem of uniqueness of extremal Käher metrics}

Following notations in Subsection 6.2. Suppose $\phi_{1}$ is also a constant scalar curvature metric. Then

$$
E\left(\phi_{0}\right)=E\left(\phi_{1}\right)=A
$$

Since

$$
\left|\partial \bar{\partial} \phi^{(l)}\right|_{\Sigma^{(l)} \times M} \leq C
$$

holds uniformly (independent of $l$ ), there exists a subsequence of $\varphi^{(l)}$ which converges to $\underline{\phi} \in \overline{\mathcal{H}}$ in the weak $C^{1,1}$ norm. Following Theorem 6.2.1 for any point $(s, t) \in \Sigma_{1}{ }^{0}$, we have

$$
\lim _{l \rightarrow \infty} \mathbf{E}\left(\phi^{(l)}(s, t)\right)=A=\inf _{\phi \in \mathcal{H}} \mathbf{E}(\phi) .
$$

In the discussion below, we fix an aribitrary interior point $(s, t) \in \Sigma_{1}{ }^{0}$. Theorem [7.1.1 implies that $\omega_{\phi^{(l)}}^{n}(s, t, \cdot)$ converges strongly to $\omega_{\phi}^{n}(s, t, \cdot)$. By Theorem 7.2.4, we have $\frac{\omega_{\underline{\phi}}^{n}(s, t, \cdot)}{\omega^{n}}>\epsilon_{0}$ as long as it is positive. The set of points where the volume ratio $\frac{\omega_{\phi}^{n}(s, t, \cdot)}{\omega^{n}}$ vanishes must have measure 0. Otherwise, it contradicts with the fact that $\sqrt{\frac{\omega_{\phi}^{n}(s, t, \cdot)}{\omega^{n}}}$ is in $W^{1,2}(M, \omega)$ (cf. Theorem 7.3.1). Thus,

$$
\frac{\omega_{\phi}^{n}(s, t, \cdot)}{\omega^{n}}>\epsilon_{0}
$$

for all points in $M$ except at most a set of measure 0 . Normalizing this volume ratio in the $L^{2}$ sense, we obtain

$$
\frac{\omega_{\phi}^{n}(s, t, x)}{\omega^{n}}>\epsilon_{0}, \forall x \in M .
$$

Since $\underline{\phi}$ has uniform $C^{1,1}$ bound, this implies that

$$
\omega_{\underline{\phi}}(s, t, \cdot)>c_{0} \omega
$$

for some positive constant $c_{0}$. In other words, the metric $g_{\underline{\phi}}$ is equivalent to $g_{0}$. For any locally supported test function $\xi$, we have ${ }^{27}$

$$
\int_{M} \log \frac{\omega_{\phi}^{n}}{\omega^{n}} \sqrt{-1} \partial \bar{\partial} \xi \wedge \omega_{\underline{\phi}}^{[n-1]}=\int_{M} \xi\left(\operatorname{Ric}\left(\omega_{0}\right)-\omega_{\underline{\phi}}\right) \wedge \omega_{\underline{\phi}}^{[n-1]} .
$$

\footnotetext{
${ }^{27}$ In any fixed open set, $\omega_{\underline{\phi}}$ can be approximated by a sequence of smooth Kähler metrics such that all Kähler metrics in the sequence has $\bar{a}$ uniform positive lower bound. Thus one can do small deformations in the arbitrary direction. Consequently, one can establish the Euler-Lagrange equation in the weak sense.
} 
Write

$$
\omega_{\underline{\phi}}=\left(g_{\alpha \bar{\beta}}+\frac{\partial^{2} \underline{\phi}}{\partial w_{\alpha} \partial w_{\bar{\beta}}}\right) d w^{\alpha} d w^{\bar{\beta}}=g_{\underline{\phi}, \alpha \bar{\beta}} d w^{\alpha} d w^{\bar{\beta}}
$$

and

$$
f=\frac{\omega_{\phi}^{n}}{\omega^{n}}
$$

Since $\log f$ is in $W^{1,2}$, we then have

$$
-\int_{M} g_{\underline{\phi}}^{\alpha \bar{\beta}} \frac{\partial \log f}{\partial w_{\alpha}} \frac{\partial \xi}{\partial w^{\bar{\beta}}} f=-\int_{M} \xi\left(\operatorname{Ric}\left(\omega_{0}\right)-\omega_{\underline{\phi}}\right) \wedge \omega_{\underline{\phi}}^{n-1}
$$

for any locally supported test function $\xi$. Hence $\log f$ satisfies the following 2 nd order non-linear equation weakly:

$$
\frac{1}{f} \frac{\partial}{\partial w_{\bar{\beta}}}\left(g^{\underline{\phi}, \alpha \bar{\beta}} f \frac{\partial}{\partial w_{\alpha}} \log f\right)=g^{\underline{\phi}, \alpha \bar{\beta}} \operatorname{Ric}\left(\omega_{0}\right)_{\alpha \bar{\beta}}-n .
$$

Note that this is a uniformly elliptic second order non-linear partial differential equation with uniformly bounded coefficients, while the right hand side is in $L^{\infty}$. According to the Holdier estimate (due to De Giorgi), there exists a small constant $\alpha \in(0,1)$ such that $\log f \in C^{\alpha}$ for any interior points. Since $\partial M=\emptyset$, this implies that $f$ is $C^{\alpha}(M)$. Appealing to the Monge-Ampere equation

$$
\operatorname{det}\left(g_{\alpha \bar{\beta}}+\frac{\partial^{2} \underline{\phi}}{\partial w_{\alpha} \partial w_{\bar{\beta}}}\right)=f,
$$

it follows that $\underline{\phi} \in C^{2, \alpha}$. Returning to the original equation of divergence form, we have

$$
g_{\underline{\phi}}^{\alpha \bar{\beta}} \frac{\partial^{2}}{\partial w_{\alpha} \partial \bar{w}_{\beta}}(\log f)=\left(g_{\underline{\phi}}^{\alpha \bar{\beta}} \operatorname{Ric}_{\alpha \bar{\beta}}(\omega)-n\right) f .
$$

Here the left hand side is a uniformly elliptic operator with $C^{\alpha}$ coefficients, the right hand side is also $C^{\alpha}$ continuous. The standard elliptic regularity theory implies that $\log \frac{\omega_{\phi}^{n}}{\omega^{n}} \in C^{2, \alpha}$. This in turn implies that $\phi \in C^{4, \alpha}$ or the right hand side is in $C^{2, \alpha} \ldots$. Repeated boot-strapping between these two equations shows that $\phi$ is smooth. Consequently, it must be of constant scalar curvature. It is easy to see $\frac{\partial \phi}{\partial s}=0$, and $\underline{\phi}(s, t)(0 \leq t \leq 1)$ satisfies the geodesic equation:

$$
\frac{\partial^{2} \underline{\phi}}{\partial t^{2}}-g_{\underline{\phi}, \alpha \bar{\beta}} \eta^{\alpha} \eta^{\bar{\beta}}=\triangle_{z} \underline{\phi}-g_{\underline{\phi}, \alpha \bar{\beta}} \eta^{\alpha} \eta^{\bar{\beta}}=0
$$

where

$$
\eta^{\alpha}=-g_{\underline{\phi}}^{\alpha \bar{\beta}} \frac{\partial^{2} \underline{\phi}}{\partial w_{\alpha} \partial \bar{w}_{\beta}}
$$


The second variation of the K energy must be identically 0 in the direction of $t$, which implies

$$
\int_{0}^{1} d t \int_{M}\left|\frac{\partial \eta^{\alpha}}{\partial w_{\bar{\beta}}}\right|_{\underline{\phi}}^{2} \omega_{\underline{\phi}}^{[n]}=0
$$

or

$$
\frac{\partial \eta^{\alpha}}{\partial w_{\bar{\beta}}} \equiv 0, \quad \forall \alpha, \beta=0, \forall 1,2, \cdots n
$$

Thus, this path represents a path of holomorphic transformation. The uniqueness is then proved.

Theorem 8.0.2. In any Kähler class, the extremal Kähler metric is unique up to holomorphic transformation.

This concludes our proof of Theorem 1.1.1.

\section{Appendix: Loop space of $G l(n, c)$ and holomorphic discs}

The purpose of this appendix is to give a proof for Lemma 3.3.1. The lemma is more or less known to experts in the field, although it is difficult to find exact statement in literatures. The proof presented here is shown to us by Professor E. Lupercio. It uses some standard theory of loop groups. We will be very explicit in our presentation here for the sake of completeness.

To simplify the following explanation, let us suppose that $G=\mathrm{GL}_{n}(\mathbf{C})$. The loop group $\mathcal{L} G$ of a Lie group $G$ is the space of maps from the unit circle $S^{1}$ in $\mathbf{C}$ to the corresponding group $G$. In this note, the space $\mathcal{L} G$ is endowed with the structure of an infinite dimensional polarized manifold. By a polarization of a vector space $H$, we mean a class of decompositions $H_{+} \oplus H_{-}$ that differ only "by a finite amount." A manifold is polarized if its tangent bundle is polarized at every fiber.

There are several important subgroups of the loop group that deserve consideration. The first of them is the subgroup $\mathcal{L}^{+} \mathrm{GL}_{n}(\mathbf{C})$ of loops $\gamma \in \mathcal{L} \mathrm{GL}_{n}(\mathbf{C})$ that extend to holomorphic maps of the closed unit disc $D^{2}$ on the complex plane $\tilde{\gamma}: D^{2} \rightarrow \mathrm{GL}_{n}(\mathbf{C})$.

The loop group has very important homogeneous spaces that posses very nice geometrical interpretations. The most important of them is the restricted Grassmannian of a Hilbert space $H$. The fundamental idea is that the loop group acts transitively on the restricted Grassmannian, and the stabilizer of a point is the subgroup $\mathcal{L}^{+} \mathrm{GL}_{n}(\mathbf{C})$. This action thus realizes the restricted Grassmannian as a homogeneous space for the loop group of the form $\mathcal{L} \mathrm{GL}_{n}(\mathbf{C}) / \mathcal{L}^{+} \mathrm{GL}_{n}(\mathbf{C})$. To define this Grassmannian, we need the concept of Polarization for the Hilbert space. Let just say that if we realize our Hilbert space as the space of functions on the circle $H^{(n)}=$ $L^{2}\left(S^{1}, \mathbf{C}^{n}\right) \cong H \otimes \mathbf{C}^{n}$, then the natural polarization for $H^{(n)}$ is given by

$$
H^{(n)}=H_{+}^{(n)} \oplus H_{-}^{(n)}
$$


where $H_{+}^{(n)}$ consists of those elements of $H^{(n)}$ that are boundary values for a holomorphic map on the unit disc $D^{2}$, and $H_{-}^{(n)}$ is the orthogonal complement of $H_{+}^{(n)}$ in $H^{(n)}$. In other words $H_{+}^{(n)}$ is the space of functions $f(z)$ so that in its Fourier expansion no negative powers of $z$ appear.

We define the restricted Grassmannian $\operatorname{Gr}\left(H^{(n)}\right)$ of $H^{(n)}$ to be the space of all closed subspaces $W$ of $H^{(n)}$ so that the projections $W \rightarrow H_{+}^{(n)}$ and $W \rightarrow H_{-}^{(n)}$ are respectively a Fredholm and a Hilbert-Schmidt operator. This definition is crafted in such a way that $W$ is then 'comparable' in a suitable sense with $H^{(n)}$ That is to say, the decomposition $W \oplus W^{\perp}$ is also a polarization. With this definition, the restricted Grassmannian is an infinite dimensional complex manifold with charts modeled on the Hilbert space $\mathcal{I}_{2}\left(W ; W^{\perp}\right)$ of Hilbert-Schmidt operators $W \rightarrow W^{\perp}$. This shows that the restricted Grassmannian group is a polarized manifold.

More relevant to this discussion is the Grassmannian $\mathrm{Gr}^{(n)} \subseteq \operatorname{Gr}\left(H^{(n)}\right)$ consisting of those $W \in \operatorname{Gr}\left(H^{(n)}\right)$ so that $z W \subseteq W$. The index of the projection $W \rightarrow H_{+}^{(n)}$ is called the virtual dimension of $W$. The virtual dimension index the connected components of the Grassmannian, that is to say that it can be thought as an isomorphism $\pi_{0} \operatorname{Gr}\left(H^{(n)}\right) \cong \mathbf{Z}$.

If we let the loop group $\mathcal{L} \mathrm{GL}_{n}(\mathbf{C})$ acts on $H^{(n)}$ by matrix multiplication (every element $\gamma(z)$ of the loop group is a matrix valued function on $S^{1}$ ), then the action induces a corresponding action on $\mathrm{Gr}^{(n)}$ - this is the purpose of the condition $z W \subseteq W$ in the definition of this Grassmannian. The action is transitive and the isotopy group of $H_{+}^{(n)}$ is precisely $\mathcal{L}^{+} \mathrm{GL}_{n}(\mathbf{C})$. This produces the identification $\mathcal{L} \mathrm{GL}_{n}(\mathbf{C}) / \mathcal{L}^{+} \mathrm{GL}_{n}(\mathbf{C}) \cong \mathrm{Gr}^{(n)}$. A version of the maximum modulus principle furthermore implies that

$$
\Omega U_{n} \cong \mathcal{L} U_{n} / U_{n} \cong \mathcal{L} \mathrm{GL}_{n}(\mathbf{C}) / \mathcal{L}^{+} \mathrm{GL}_{n}(\mathbf{C}) \cong \mathrm{Gr}^{(n)}
$$

There is natural stratification of $\operatorname{Gr}^{(n)}$ whose strata are indexed by homomorphisms $S^{1} \rightarrow$ $\mathrm{GL}_{n}(\mathbf{C})$. Every such homomorphism can be written in the form

$$
z^{\mathbf{k}}=\left(\begin{array}{ccccc}
z^{k_{1}} & & & & \\
& z^{k_{2}} & & & \\
& & z^{k_{3}} & & \\
& & & \ddots & \\
& & & & z^{k_{n}}
\end{array}\right)
$$

where $\mathbf{k}=\left(k_{1}, k_{2}, k_{3}, \ldots, k_{n}\right)$ is an integer partition of the non-negative integer number $k$, namely $k_{1}+k_{2}+\cdots k_{n}=k$.

The Birkhoff factorization theorem ${ }^{28}$ establishes that any loop $\gamma(z) \in \mathcal{L G L}_{n}(\mathbf{C})$ can be factored in the form

$$
\gamma(z)=\gamma_{-}(z) z^{\mathbf{k}} \gamma_{+}(z)
$$

\footnotetext{
${ }^{28}$ The Birkhoff factorization theorem is equivalent to the theorem of Grothendieck that states that every holomorphic bundle of rank $n$ over the Riemman sphere can be uniquely written in the form of powers of the Hopf bundle $O\left(k_{1}\right) \oplus \cdots \oplus O\left(k_{n}\right)$.
} 
where $\gamma_{+}(z), \gamma_{-}(1 / z) \in \mathcal{L}^{+} \mathrm{GL}_{n}(\mathbf{C})$ and $\mathbf{k}$ is well defined up to the ordering of the $k_{i}$ 's.

We will say that $\mathbf{k}$ is the multi-index (or Grothendieck index) of $\gamma(z)$ whenever equation 9.3 holds. We will tolerate the ordering ambiguity in this definition.

Let us return to the description of the stratification of $\mathrm{Gr}^{(n)}$. Notice that given a loop $\gamma(z) \in \mathcal{L} \mathrm{GL}_{n}(\mathbf{C})$ of index $\mathbf{k}$, then multiplying on the right by any element $\phi_{+}(z) \in \mathcal{L}^{+} \mathrm{GL}_{n}(\mathbf{C})$ will not affect the multi-index, that is, $\gamma(z) \phi_{+}(z)$ still has multi-index $\mathbf{k}$. ¿From this we conclude that the multi-index is constant along orbits of the right-action of $\mathcal{L}^{+} \mathrm{GL}_{n}(\mathbf{C})$ in $\mathcal{L G L}_{n}(\mathbf{C})$. In other words every point of $\mathrm{Gr}^{(n)}$ has a well defined multi-index.

Let us define $\mathcal{L}^{-} \mathrm{GL}_{n}(\mathbf{C})$ by declaring that $\phi_{-}(z) \in \mathcal{L}^{-} \mathrm{GL}_{n}(\mathbf{C})$ if and only if $\phi_{-}(1 / z) \in$ $\mathcal{L}^{+} \mathrm{GL}_{n}(\mathbf{C})$. Again the action of $\mathcal{L}^{-} \mathrm{GL}_{n}(\mathbf{C})$ doesn't affect te multi-index of an element. Then the orbits of the action of $\mathcal{L}^{-} \mathrm{GL}_{n}(\mathbf{C})$ in $\mathrm{Gr}^{(n)}$ are precisely the same as the sets of elements in with the same multi-index and all its permutations. This is once more a consequence of the Birkhoff factorization. To avoid the problem of the permutations we will have to consider a smaller subgroup $N^{-}$of $\mathcal{L}^{-} \mathrm{GL}_{n}(\mathbf{C})$. The group $N^{-}$consists of those elements in $\gamma_{-} \in \mathcal{L}^{-} \mathrm{GL}_{n}(\mathbf{C})$ so that,

$$
\gamma_{-}(\infty)=\left(\begin{array}{cccc}
1 & * & * & * \\
0 & 1 & * & * \\
0 & 0 & \ddots & * \\
0 & 0 & 0 & 1
\end{array}\right)
$$

For every partition $\mathbf{k}$ of $k$ (and here the order is important) we define the subspaces $H_{\mathbf{k}} \in \mathrm{Gr}^{(n)}$ as

$$
H_{\mathbf{k}}=z^{\mathbf{k}} H_{+}^{(n)}=\left\{f(z)=\left(f_{1}(z), \ldots, f_{n}(z)\right): f_{i}(z)=\sum_{j=k_{i}}^{\infty} a_{j}^{i} z^{j}, a_{j}^{i} \in \mathbf{C}\right\} .
$$

and we define $\Sigma_{\mathbf{k}}^{\sigma}$ to be the orbit of $H_{\mathbf{k}}$ under the action of $\mathcal{L}^{-} \mathrm{GL}_{n}(\mathbf{C})$ in $\mathrm{Gr}^{(n)}$.

Define now $\Sigma_{\mathbf{k}}$ to be $N^{-} \cdot H_{\mathbf{k}} \subset \operatorname{Gr}^{(n)}$ (again, the order in $\mathbf{k}$ in important.). We have

Proposition 9.0.3. The set of all elements in $\mathrm{Gr}^{(n)}$ of multi-index $\mathbf{k}$ is precisely $\Sigma_{\mathbf{k}}^{\sigma}$.

Proof. Take any element $\gamma(z) \in \mathcal{L} \mathrm{GL}_{n}(\mathbf{C})$ of index $\mathbf{k}$, and write $W=\gamma(z) H_{+}^{(n)} \in \mathrm{Gr}^{(n)}$. Using the Birkhoff factorization $\gamma(z)=\gamma_{-}(z) z^{\mathbf{k}} \gamma_{+}(z)$, we have $W=\gamma_{-}(z) z^{\mathbf{k}} \gamma_{+}(z) H_{+}^{(n)}=\gamma(z)=$ $\gamma_{-}(z) z^{\mathbf{k}} H_{+}^{(n)}=\gamma_{-}(z) H_{\mathbf{k}}$. It follows that every $W$ is in some $\Sigma_{\mathbf{k}}^{\sigma}$.

In fact, a more refined statement is true.

Lemma 9.0.4. For every $W \in \mathrm{Gr}^{(n)}$, there is a $\gamma_{-}(z) \in N^{-}$and an $\mathbf{k}$ so that $W=\gamma_{-}(z) H_{\mathbf{k}}$.

Proof. We use the Pressley-Segal identification of $H^{(n)}=L^{2}\left(S^{1}, \mathbf{C}^{n}\right) \rightarrow L^{2}\left(S^{1}, \mathbf{C}\right)=H$ given by $\left(f_{1}, \ldots, f_{n}\right) \mapsto \widetilde{f}(\zeta)=\sum_{i=1}^{n} \zeta^{i-1} f_{i}\left(\zeta^{n}\right)$. We define $\check{W}=\bigcup_{m} W \cap \zeta^{m} H_{-}$.. Choose an algebraic 
vector space basis of $\check{W}$ by considering the subset of $\check{W}$ consisting of elements of the form $\zeta^{s}+\sum_{k=-\infty}^{s-1} a_{k} \zeta^{k}$, and choosing one such element for each possible value of $s$. Call $S$ the set of all the values of $s$ appearing in this construction. Denote by $w_{s}=\zeta^{s}+\sum_{k=-\infty}^{s-1} a_{k} \zeta^{k}$ the chosen element so that our basis is $\mathcal{B}=\left\{w_{s}: s \in S\right\}$.. Let $H_{S}=\left\{\sum_{s \in S} a_{s} \zeta^{s}\right\}$ be the Hilbert space generated by the $\zeta^{s}$. We may suppose that the orthogonal projection $W \rightarrow H_{S}$ sends $w_{s} \mapsto \zeta^{s}$ (by using reduction of the basis $\mathcal{B}$ to its reduced echelon form.). This induces an isomorphism $W \cong H_{S}$. Since $z W \subset W$, then $s \in S$ implies $s+n \in S$ (cf. p. 98 in [9]). There are $n$ elements $r_{i}$ in $S$ so that $r_{i}-n$ is not in $S$, and $S=\bigcup_{q \in \mathbf{N}}\left\{r_{1}+q n, \ldots, r_{n}+q n\right\}$. Writing $r_{i}=n k_{i}+i-1$ we conclude immediately that $H_{S}=H_{\mathbf{k}}$.

We can find the element $\gamma_{-}(z)$ we are seeking for using the isomorphism $W \rightarrow H_{\mathbf{k}}$ by the following procedure. We define the smooth function $v_{i}(z) \in W$ to be the element in $W$ that projects to $\left(0, \ldots, z^{k_{i}}, \ldots, 0\right)=\zeta^{i-1+n k_{i}}=\zeta^{r_{i}}$, so that we can write a basis of $W$ as $\mathcal{B}^{\prime}=\left\{z^{k} v_{i}(z): 1 \leq n, k \geq 0\right\}$. The matrix of smooth functions $v(z)=\left(v_{1}(z), \ldots, v_{n}(z)\right)$ defines an element in $\mathcal{L G L}_{n}(\mathbf{C})$. Clearly $v(z) \cdot H_{+}=\mathrm{W}$. Define $\gamma_{-}(z)=v(z) \cdot z^{-\mathbf{k}}$. Then $\gamma_{-}(z) \cdot H_{\mathbf{k}}=W$. Since $v(z)$ projects to $\left(0, \ldots, z^{k_{i}}, \ldots, 0\right) \in H_{\mathbf{k}}$ then $\gamma_{i}(z)=v_{i}(z) z^{-k_{i}}$ projects to $(0, \ldots, 1, \ldots, 0) \in H_{+}$, therefore no positive powers of $z$ appear in th expansion of $\gamma_{-}(z)$ and moreover the constant term in the Laurent expansion $\gamma_{-}(\infty)$ is upper triangular . We also have that $\operatorname{deg} \operatorname{det} \gamma_{-}(z)+v \cdot \operatorname{dim} W=v \cdot \operatorname{dim} H_{\mathbf{k}}$, and therefore $\operatorname{deg} \operatorname{det} \gamma_{-}(z)=0$. From this we conclude that $\gamma_{-}(z) \in N^{-}$.

Proposition 9.0.5. The Grassmannian $\mathrm{Gr}^{(n)}$ admits a partition

$$
\operatorname{Gr}^{(n)}=\coprod_{\mathbf{k}} \Sigma_{\mathbf{k}}^{\sigma}
$$

Moreover, each $\Sigma_{\mathbf{k}}^{\sigma}$ is the union of the $\Sigma_{\mathbf{k}}$ 's for all permutations in the order of $\mathbf{k}$, namely

$$
\Sigma_{\mathbf{k}}^{\sigma}=\coprod_{\epsilon \in S_{n}} \Sigma_{\epsilon(\mathbf{k})}
$$

Proof. It is easy to see that for each permutation $\epsilon$ we have that $H_{\epsilon(\mathbf{k})} \in \Sigma_{\mathbf{k}}^{\sigma}$ and hence $\Sigma_{\mathbf{k}}^{\sigma} \supseteq$ $\bigcup_{\epsilon \in S_{n}} \Sigma_{\epsilon(\mathbf{k})}$. Since the whole $\mathrm{Gr}^{(n)}$ is the disjoint union of $\Sigma_{\mathbf{j}}$ for all possible $\mathbf{j}$ and since $N^{-} \subset$ $\mathcal{L}^{-} \mathrm{GL}_{n}(\mathbf{C})$, it is enough to show that $\Sigma_{\mathbf{k}}^{\sigma}$ does not contain any $H_{\mathbf{j}}$ for a $\mathbf{j}$ that is not a permutation of $\mathbf{k}$. To see this, we associate a sequence $\omega(W)$ to each $W$ by $\omega_{i}(W)=\operatorname{dim}\left(W \cap \zeta^{i} H_{-}\right)$. It is immediate to see that the sequence $\omega$ is $\mathcal{L}^{-} \mathrm{GL}_{n}(\mathbf{C})$-invariant, and it nevertheless distinguishes $H_{\mathbf{k}}$ from $H_{\mathbf{j}}$ (because of the proof of the previous proposition we can recover the ordered multiindex from $\omega$.)

Finally, we are ready to restate Lemma 3.3.1 here for the convenience of readers.

Theorem 9.0.6. The set $\Sigma_{\mathbf{k}}$ is a contractible submanifold of $\mathrm{Gr}^{(n)}$ of codimension

$$
c d(\mathbf{k})=\sum_{i<j}\left|k_{i}-k_{j}\right|-\varrho(\mathbf{k}),
$$


where $\varrho(\mathbf{k})$ is the number of inversions of $\mathbf{k}$.

Proof. Let us define an open neighborhood of $H_{\mathbf{k}}$. Let $L_{0}^{-} \subset \mathcal{L}^{-} \mathrm{GL}_{n}(\mathbf{C})$ be is the subgroup of elements $\gamma(z)$ so that $\gamma(\infty)$ is the identity matrix. Consider the map $\mathcal{L G L}_{n}(\mathbf{C}) \rightarrow \operatorname{Gr}^{(n)}: \gamma(z) \mapsto$ $\gamma(z) \cdot H_{\mathbf{k}}$. Let $U_{\mathbf{k}}$ be the image under this map of $z^{\mathbf{k}} L_{0}^{-} z^{-\mathbf{k}}$. Clearly $H_{\mathbf{k}} \in U_{\mathbf{k}}$.

Now $U_{\mathbf{k}}$ we prove that is an open set. Here we return to the proof of Lemma 9.0.4 (1?). When we proved that $\gamma_{-}(z) \in N^{-}$we should point out that the same argument actually proves a little bit more. Indeed we have that $z^{-\mathbf{k}} v_{i}(z) z^{\mathbf{k}} \in H$ is of the form $(0, \ldots, 1, \ldots, 0)+h_{-}(z) \in H$ where $h_{-}(z) \in H_{-}$. Therefore $\gamma_{-}(z) \in z^{\mathbf{k}} L_{0}^{-} z^{-\mathbf{k}}$. Without loss of generality we assume $\mathbf{k}=(0, \ldots, 0)$, otherwise we shift by the appropiate $z^{\mathbf{k}}$. In this case the Birkhoff factorization can be refined to state that every loop $\gamma(z)$ factorizes uniquely as $\left.\gamma_{(} z\right) \gamma_{+}(z)$ where $\gamma_{-}(z) \in L_{0}^{-}$and $\gamma_{+}(z) \in$ $\mathcal{L}^{+} \mathrm{GL}_{n}(\mathbf{C})$. This implies that $L_{0}^{-} \cong U_{\mathbf{0}}$ is an open chart of $\mathrm{Gr}^{(n)}=\mathcal{L G L}_{n}(\mathbf{C}) / \mathcal{L}^{+} \mathrm{GL}_{n}(\mathbf{C})$, and therefore $U_{\mathbf{k}}$ is open. Also notice that this argument also implies that $\Sigma_{\mathbf{k}} \subseteq U_{\mathbf{k}}$.

We want to show then that the codimension of the inclusion $\Sigma_{\mathbf{k}} \subseteq U_{\mathbf{k}}$ is given by the formula of the statement of the theorem. Since we know that in the proof of Lemma 1 we actually have $\gamma_{-}(z) \in\left(z^{\mathbf{k}} L_{0}^{-} z^{-\mathbf{k}}\right) \cap N^{-}$. In fact $\mathcal{L} \mathrm{GL}_{n}(\mathbf{C}) \rightarrow \mathrm{Gr}^{(n)}: \gamma(z) \mapsto \gamma(z) \cdot H_{\mathbf{k}}$ induces an identification $\left(N^{-} \cap z^{\mathbf{k}} L_{0}^{-} z^{\mathbf{k}}\right) \rightarrow \Sigma_{\mathbf{k}}$. We claim that the multiplication in $\mathcal{L} \mathrm{GL}_{n}(\mathbf{C})$ indices an identification $\left(N^{-} \cap z^{\mathbf{k}} L_{0}^{-} z^{-\mathbf{k}}\right) \times\left(N^{+} \cap z^{\mathbf{k}} L_{0}^{-} z^{-\mathbf{k}}\right) \rightarrow z^{\mathbf{k}} L_{0}^{-} z^{-\mathbf{k}}=U_{\mathbf{k}}$, where $N^{+}$is just as $N^{-}$except that we talk of lower triangular matrices.

All that remains then is to compute the dimension of $N^{+} \cap z^{\mathbf{k}} L_{0}^{-} z^{-\mathbf{k}}$. This is done as follows. By taking Laurent expansions of the entries of en element $\gamma(z) \in\left(N^{+} \cap z^{\mathbf{k}} L_{0}^{-} z^{-\mathbf{k}}\right)$ we conclude that for $i<j$ then $\gamma_{i i}(z)=1, \gamma_{i j}(z)=\sum_{l=1}^{k_{i}-k_{j}-1} a_{l} z^{l}$ and $\gamma_{j i}=\sum_{l=0}^{k_{j}-k_{i}-1} a_{l} z^{l}$. By counting coefficients we obtain the desired formula.

Remark. Notice that the proof of the previous theorem actually shows more. It shows that the codimension of elements $\gamma(z)$ of multi-index $\mathbf{k}$ inside $\mathcal{L G L}_{n}(\mathbf{C})$ is given by the same formula. This is done by considering $\left(N^{-} \cap z^{\mathbf{k}} L_{0}^{-} z^{-\mathbf{k}}\right) \times\left(N^{+} \cap z^{\mathbf{k}} L_{0}^{-} z^{-\mathbf{k}}\right) \times \mathcal{L}^{+} \mathrm{GL}_{n}(\mathbf{C})$ instead of $\left(N^{-} \cap z^{\mathbf{k}} L_{0}^{-} z^{-\mathbf{k}}\right) \times\left(N^{+} \cap z^{\mathbf{k}} L_{0}^{-} z^{-\mathbf{k}}\right)$.

\section{References}

[1] S. Bando and T. Mabuchi. Uniqueness of Einstein Kaehler metrics modulo connected group actions. Algebraic geometry, Sendai, 1985, Adv. Stud. Pure Math., 10 (1987), 11-40.

[2] E.D. Bedford and T.A. Taylor. The Drichelet problem for the complex Monge-Ampere operator.. Invent. Math., 37 (1976), 1-44.

[3] E. Calabi. An extension of E. Hopf's Maximum principle with an application to Riemannian geometry. Duke Math. J., 25 (1957), 45-56. 
[4] E. Calabi. Extremal Kähler metrics. In Seminar on Differential Geometry, Ann. of Math. Studies, University Press, Series 102 (1982), Princeton, 259-290.

[5] E. Calabi. Extremal Kähler metrics, II. Differential geometry and Complex analysis, 96-114, Springer, 1985.

[6] E. Calabi and X. X. Chen. Space of Kähler metrics and Calabi flow. Journal of Differential Geometry, 2002.

[7] X. X. Chen. On lower bound of the Mabuchi energy and its application. International Mathematics Research Notices, 12 (2000), .

[8] X. X. Chen. Space of Kähler metrics. Journal of Differential Geometry, 56 (2000), 189-234.

[9] R. Cohen, E. Lupercio and G. Segal. Holomorphic spheres in loop groups and Bott periodicity. Surveys in Differential Geometry, 2000, 83-106.

[10] S. K. Donaldson. Symmetric spaces, Kähler geometry and Hamiltonian dynamics. Northern California Symplectic Geometry Seminar. Amer. Math. Soc. Transl., 196 (1999), 13-33.

[11] S. K. Donaldson. Holomorphic discs and the complex Monge-Ampère equation. Journal of Sympletic Geometry, 1 (2001), 171-196.

[12] S. K. Donaldson. Scalar curvature and projective embeddings. I. Journal of Differential Geometry, vol.59(3), (2001), 479-522.

[13] S. K. Donaldson. Scalar curvature and projective embeddings. II. priprint, ArXiv. math.DG/0407534, 2004.

[14] F. Fostneric. Analytic discs with boundaries in a maximal real submanifolds of $\mathbb{C}^{2}$. Ann. Inst. Fourier, Grenoble, 37 (1987), 1-44.

[15] A. Futaki. Remarks on Extremal Kähler metrics on ruled manifolds.. Nagoya Math. J., 126 (1992), 89-101.

[16] J. Globenik. Perturbation by analytic discs along maximal real submanifolds of $\mathbb{C}^{n}$. Math. Z., 217 (1994), 287-316.

[17] L. Nirenberg L. Caffarelli and J. Spruck. The Dirichlet problem for nonlinear second-order elliptic equation I, Monge-Ampere equation. Comm. Pure and Appl. Math., 37 (1984), 369-402.

[18] J.T. Kohn L. Nirenberg L. Caffarelli and J. Spruck. The Dirichlet problem for nonlinear second-order elliptic equation II. Complex Monge-Ampere equation. Comm. Pure and Appl. Math., 38 (1985), 209-252. 
[19] L. Lempert. Solving the degenerate Monge-Ampere equation with one concentrated singularity. Math. Ann., 263 (1983), 515-532.

[20] T. Mabuchi. Some symplectic geometry on compact Kähler manifolds I. Osaka, J. Math., 24 (1987), 227-252.

[21] Yong-Geun Oh. Riemann-Hilbert problem and application to the perturbation theory of analytic discs. Kyungpook Math., J., 35 (1995), 38-75.

[22] Yong-Geun Oh. Fredhom theory of holomorphic discs under the perturbation theory of boundary conditions. Math. Z., 222 (1996), 505-520.

[23] Robert Osserman. On the inequality $\Delta u \geq f(u)$. Pacific J. Math., 7 (1957), 1641-1647.

[24] D. H. Phong and Jacob Sturm. Stability, Energy Functionals, and Khler-Einstein Metrics . Comm. Anal. Geom., 11, vol 3 (2003), 565-597.

[25] S. Paul and G. Tian. Algebraic and analytic K-stability. preprint(2004), math.DG/0405530.

[26] S. Semmes. Complex Monge-Ampère equations and sympletic manifolds. Amer. J. Math., 114 (1992), 495-550.

[27] G. Tian. Kähler-Einstein metrics with positive scalar curvature. Invent. Math., 130 (1997), $1-39$.

[28] G. Tian. Canonical metrics in Kähler geometry. Lectures in Mathematics ETH Zrich (Notes taken by Meike Akveld), Birkhauser, 2000.

[29] G. Tian On Calabis conjecture for complex surfaces with positive first Chern class, Invent. Math, 130(1990), 1-37.

[30] G. Tian. Bott-Chern forms and geometric stability. Discrete Contin. Dynam. Systems, 6 (2000), no. 1, 211-220.

[31] G. Tian and X. H. Zhu. A new holomorphic invariant and uniqueness of Kähler-Ricci solitons. Comment. Math. Helv., 77 (2002), no. 2, 297-325.

[32] N. P. Vekuba. Systems of Singular Integral Equations. Groningen, Nordhoff, 1967.

[33] S. T. Yau. On the Ricci curvature of a compact Kähler manifold and the complex MongeAmpere equation, I* . Comm. Pure Appl. Math.,/ 31 (1978), 339-441.

X. X. Chen, Department of Mathematics, University of Wisconsin. xiu@math.wisc.edu

G. Tian, Department of Mathematics, Princeton University and Beijing University. tian@math.princeton.edu 